\title{
Neutrino Masses and Leptogenesis in Left-Right Symmetric Models: A Review From a Model Building Perspective
}

\author{
Chandan Hati ${ }^{1 *}$, Sudhanwa Patra ${ }^{2}$, Prativa Pritimita ${ }^{3}$ and Utpal Sarkar ${ }^{1,2,3,4}$ \\ ${ }^{1}$ Laboratoire de Physique Corpusculaire, Centre National de la Recherche Scientifique/IN2P3 UMR 6533, Aubière, France, \\ ${ }^{2}$ Department of Physics, Indian Institute of Technology Bhilai, Chhattisgarh, India, ${ }^{3}$ Center of Excellence in Theoretical and \\ Mathematical Sciences, Siksha 'O' Anusandhan University, Bhubaneswar, India, ' Department of Physics, Indian Institute of \\ Technology Kharagpur, Kharagpur, India
}

OPEN ACCESS

Edited by:

Alexander Merle

Max Planck Institute for Physics

(MPG), Germany

Reviewed by:

Eduardo Peinado,

Instituto de Física, Universidad

Nacional Autónoma de México,

Mexico

Bhupal Dev,

Washington University in St. Louis,

United States

*Correspondence:

Chandan Hati

chandan.hati@clermont.in2p3.fr

Specialty section:

This article was submitted to

High-Energy and Astroparticle

Physics,

a section of the journal

Frontiers in Physics

Received: 17 October 2017

Accepted: 14 February 2018

Published: 06 March 2018

Citation:

Hati C, Patra S, Pritimita $P$ and Sarkar U (2018) Neutrino Masses and Leptogenesis in Left-Right Symmetric

Models: A Review From a Model

Building Perspective.

Front. Phys. 6:19

doi: 10.3389/fphy.2018.00019
In this review, we present several variants of left-right symmetric models in the context of neutrino masses and leptogenesis. In particular, we discuss various low scale seesaw mechanisms like linear seesaw, inverse seesaw, extended seesaw and their implications to lepton number violating process like neutrinoless double beta decay. We also visit an alternative framework of left-right models with the inclusion of vector-like fermions to analyze the aspects of universal seesaw. The symmetry breaking of left-right symmetric model around few TeV scale predicts the existence of massive right-handed gauge bosons $W_{R}$ and $Z_{R}$ which might be detected at the $L H C$ in near future. If such signals are detected at the LHC that can have severe implications for leptogenesis, a mechanism to explain the observed baryon asymmetry of the Universe. We review the implications of TeV scale left-right symmetry breaking for leptogenesis.

Keywords: left-right symmetry, neutrino mass, leptogenesis, neutrinoless double beta decay, low scale seesaw

\section{INTRODUCTION}

Although the Standard Model (SM) of particle physics is highly successful in explaining the low energy phenomenology of fundamental particles, the reasons to believe it is incomplete are not less. The most glaring of them all is the issue of neutrino mass which has been confirmed by the oscillation experiments. Some more unsolved puzzles like dark matter, dark energy, baryon asymmetry of the universe strongly suggest that SM is only an effective limit of a more fundamental theory of interactions. In addition to the fact that gravity is completely left out in the SM, the strong interaction is not unified with weak and electromagnetic interactions. In fact, even in the electroweak "unification" one still has two coupling constants, $g$ and $g^{\prime}$ corresponding to $S U(2)_{L}$ and $U(1)_{Y}$. Thus, one is tempted to seek for a more complete theory where the couplings $g_{s}, g$, and $g^{\prime}$ unify at some higher energy scale giving a unified description of the fundamental interactions. Given that the ratio $m_{\mathrm{Pl}} / m_{W}$ is so large, where $m_{\mathrm{Pl}}=1.2 \times 10^{19} \mathrm{GeV}$ is the Planck scale, another major issue in the SM is the infamous "hierarchy problem." The discovery of the Higgs boson with a mass around $125 \mathrm{GeV}$ has the consequence that, if one assumes the Standard Model as an effective 
theory, then $\lambda \sim \mathcal{O}(0.1)$ and $\mu^{2} \sim(\mathcal{O}(100) \mathrm{GeV})^{2}$ (including the effects of 2-loop corrections). The problem is that every particle that couples, directly or indirectly, to the Higgs field yields a correction to $\mu^{2}$ resulting in an enormous quantum correction. For instance, let us consider a one-loop correction to $\mu^{2}$ coming from a loop containing a Dirac fermion $f$ with mass $m_{f}$. If $f$ couples to the Higgs boson via the coupling term $\left(-\lambda_{f} \phi \bar{f} f\right)$, then the correction coming from the one-loop diagram is given by

$$
\Delta \mu^{2}=\frac{\lambda_{f}^{2}}{8 \pi^{2}} \Lambda_{\mathrm{UV}}^{2}+\cdots,
$$

where $\Lambda_{\mathrm{UV}}$ is the ultraviolet momentum cutoff and the ellipsis are the terms proportional to $m_{f}^{2}$, growing at most logarithmically with $\Lambda_{\mathrm{UV}}$. Each of the quarks and leptons in the SM plays the role of $f$, and if $\Lambda_{\mathrm{UV}}$ is of the order of $m_{\mathrm{Pl}}$, then the quantum correction to $\mu^{2}$ is about 30 orders of magnitude larger than the required value of $\mu^{2}=92.9 \mathrm{GeV}^{2}$. Since all the SM quarks, leptons, and gauge bosons obtain masses from $\langle\phi\rangle$, the entire mass spectrum of the Standard Model is sensitive to $\Lambda_{\mathrm{UV}}$. Thus, one expects some new physics between $m_{W}$ and $m_{\mathrm{Pl}}$ addressing this problem. There are also other questions such as why the fermion families have three generations; is there any higher symmetry that dictates different fermion masses even within each generation; in the CKM matrix the weak mixing angles and the $\mathrm{CP}$ violating phase are inputs of the theory, instead of being predicted by the SM. Finally, in the cosmic arena, the observed baryon asymmetry of the universe cannot be explained within the SM. Also there are no suitable candidates for dark matter and dark energy in the SM. These also point toward the existence of physics beyond the SM.

In this review, we study several variants of left-right symmetric models which is one of the most popular candidates for physics beyond the standard model. We will review the leftright symmetric models in the context of neutrino masses and leptogenesis. We will study various low scale seesaw mechanisms in the context of left-right symmetric models and their implications to lepton number violating process like neutrinoless double beta decay. We will also discuss an alternative framework of left-right models with the inclusion of vector-like fermions as proposed to analyze various aspects. Interestingly, the breaking of left-right symmetry around few $\mathrm{TeV}$ scale predicts the existence of massive right-handed gauge bosons $W_{R}$ and $Z_{R}$ in left-right symmetric models. These heavy gauge bosons might be detected at the LHC in near future. If such signals are detected at the LHC that can have conclusive implications for leptogenesis, a mechanism to explain the observed baryon asymmetry of the Universe. In this review we will also discuss the implications of such a detection of left-right symmetry breaking for leptogenesis in detail. Before closing this paragraph we would like to stress the fact that this review is far from comprehensive and covers only a limited variety of topics from the vast choices of LRSM-related scenarios. For example, a detailed discussion of the relevant collider phenomenology of the right-handed gauge bosons $W_{R}$ and $Z_{R}$ and the Higgs sector is beyond the scope of this review. Some relevant references for the LHC phenomenology of $W_{R}$ and heavy neutrinos are in Keung and Senjanovic [1], Nemevsek et al. [2], Das et al. [3], Chen et al. [4] and Mitra et al. [5] and for Higgs sector some relevant references are in Bambhaniya et al. [6], Dutta et al. [7], Dev et al. [8] and Mitra et al. [9].

The plan for rest of the review is as follows. In section 2 we briefly review the standard seesaw and radiative mechanisms for light neutrino mass generation. In section 3 we first introduce and then review the standard left-right symmetric theories and the implementation of different types of low scale seesaw implementations. In section 4 we review an alternative formulation of left-right symmetric theories which uses a universal seesaw to generate fermion masses. We also discuss the implications of this model for neutrinoless double beta decay in this case for the specific scenario of type II seesaw dominance. In section 5 we give a brief introduction to leptogenesis and review some of the standard leptogenesis scenarios associated with neutrino mass generation. In section 6 we review the situation of leptogenesis in left-right symmetric theories and the implications of a $\mathrm{TeV}$ scale left-right symmetry breaking for leptogenesis. Finally, in section 7 we make concluding remarks.

\section{NEUTRINO MASSES}

The atmospheric, solar and reactor neutrino experiments have established that the neutrinos have small non-zero masses which are predicted to be orders of magnitude smaller than the charged lepton masses. However, in the SM the left handed neutrinos $v_{i L}, i=e, \mu, \tau$, transform as $(1,2,-1)$ under the gauge group $S U(3)_{c} \times S U(2)_{L} \times U(1)_{Y}$. Consequently, one cannot write a gauge singlet Majorana mass term for the neutrinos. On the other hand, there are no right handed neutrinos in the SM which would allow a Dirac mass term. The simplest way around this problem is to add singlet right handed neutrinos $v_{i R}$ with the transformation $(1,1,0)$ under the SM gauge group. Then one can straightaway write the Yukawa couplings giving Dirac mass to the neutrinos

$$
-\mathcal{L}_{\text {mass }}=\frac{1}{2} h_{i j} \bar{\psi}_{i L} v_{j R} \phi,
$$

such that once $\phi$ acquires a VEV, the neutrinos get Dirac mass $m_{D i j}=h_{i j} v$. Here $\psi_{i L}$ stands for the $S U(2)_{L}$ lepton doublet. However, to explain the lightness of the neutrinos one needs to assume a very small Yukawa coupling for neutrinos in comparison to charged leptons and quarks. However, we do not have a theoretical understanding of why the Yukawa coupling should be so small. Moreover, the accidental $B-L$ symmetry of the SM forbids Majorana masses for the neutrinos. One way out is to consider the dimension-5 effective lepton number violating operator [10-13] of the form

$$
\mathcal{L}_{\operatorname{dim}-5}=\frac{\left(\nu \phi^{0}-e \phi^{+}\right)^{2}}{\Lambda},
$$

where $\Lambda$ is the scale corresponding to some new extension of the $\mathrm{SM}$ violating lepton number. This dimension- 5 term can induce small Majorana masses to the neutrinos after the eletroweak symmetry breaking

$$
-\mathcal{L}_{\text {mass }}=m_{v} v_{i L}^{T} C^{-1} v_{j L},
$$


with $m_{v}=v^{2} / \Lambda$. Here, $C$ is the charge conjugation matrix. Consequently, lepton number violating new physics at a high scale $\Lambda$ would naturally explain the smallness of neutrino masses. In what follows, we discuss some of the popular mechanisms of realizing the same.

\subsection{Seesaw Mechanism: Type-I}

The type-I seesaw mechanism ${ }^{1}[14-20]$ is the simplest mechanism of obtaining tiny neutrino masses. In this mechanism, three singlet right handed neutrinos $N_{i R}$ are added to the SM; and one can write a Yukawa term similar to Equation (2) and a Majorana mass term for the right handed neutrinos since they are singlets under the SM gauge group. The relevant Lagrangian is given by

$$
-\mathcal{L}_{\text {type-I }}=h_{i \alpha} \bar{N}_{i R} \phi l_{\alpha L} \phi+\frac{1}{2} M_{i j} N_{i L i L}^{c T} C^{-1} N_{j L}^{c}+\text { h.c.. }
$$

Note that, the Majorana mass term breaks the lepton number explicitly and since the right handed neutrinos are SM gauge singlets, there is no symmetry protecting $M_{i j}$ and it can be very large. Now after the symmetry breaking, combining the Dirac and Majorana mass matrices we can write

$$
\begin{aligned}
-2 \mathcal{L}_{\text {mass }} & =m_{D \alpha i} v_{\alpha L}^{T} C^{-1} N_{i L}^{c}+M_{i} N_{i L}^{c T} C^{-1} N_{j L}^{c}+\text { h.c. } \\
& =\left(\begin{array}{ll}
v_{\alpha} & N_{i}^{c}
\end{array}\right)_{L}^{T} C^{-1}\left(\begin{array}{cc}
0 & m_{D \alpha i} \\
m_{D \alpha i}^{T} & M_{i}
\end{array}\right)\left(\begin{array}{c}
v_{\alpha} \\
N_{i}^{c}
\end{array}\right)_{L}+\text { h.c. }
\end{aligned}
$$

where $m_{D \alpha i}=h_{D \alpha i} v$. Now assuming that the eigenvalues of $m_{D}$ are much less than those of $M$ one can block diagonalize the mass matrix to obtain the light Majorana neutrinos with masses $m_{v i j}=-m_{D \alpha i} M_{i}^{-1} m_{D \alpha i}^{T}$ and heavy neutrinos with mass $m_{N}=M_{i}$. Note that if any of the right handed neutrino mass eigenvalues $\left(M_{i}\right)$ vanish then some of the left handed neutrinos will combine with the right handed neutrinos to form Dirac neutrinos. For $n$ generations, if the rank of $M$ is $r$, then there will be $2 r$ Majorana neutrinos and $n-r$ Dirac neutrinos. The type-I seesaw mechanism not only generates tiny neutrino masses, but also provides the necessary ingredients for explaining the baryon asymmetry of the universe via leptogenesis [21], which we will discuss in length in the next section.

\subsection{Seesaw Mechanism: Type-II}

In type-II seesaw mechanism [18, 19, 22-28], the effective operator given in Equation (3) is realized by extending the SM to include an $S U(2)_{L}$ triplet Higgs $\xi$ which transforms under the SM gauge group $S U(3)_{c} \times S U(2)_{L} \times U(1)_{Y}$ as $(1,3,1)$. For simplicity we assume that there are no right handed neutrinos in this model and only one triplet scalar is present. The Yukawa couplings of the triplet Higgs with the left handed lepton doublet $\left(v_{i}, l_{i}\right)$ are given by

$$
-\mathcal{L}_{\text {type-II }}=f_{i j}\left[\xi^{0} v_{i} v_{j}+\xi^{+}\left(v_{i} l_{j}+v_{j} l_{i}\right) / \sqrt{2}+\xi^{++} l_{i} l_{j}\right] .
$$

\footnotetext{
${ }^{1}$ The seesaw mechanisms generically require a new heavy scale (as compared to the electroweak scale) in the theory, inducing a small neutrino mass (millions of times smaller than the charged lepton masses). Hence the name "seesaw."
}

Now a non-zero VEV acquired by $\xi^{0}\left(\left\langle\xi^{0}\right\rangle=u\right)$ gives Majorana masses to the neutrinos. Note that $u$ has to be less than a few $\mathrm{GeV}$ to not affect the electroweak $\rho$-parameter. The most general Higgs potential with a doublet and a triplet Higgs has the form

$$
\begin{aligned}
V= & m_{\phi}^{2} \phi^{\dagger} \phi+m_{\xi}^{2} \xi^{\dagger} \xi+\frac{1}{2} \lambda_{1}\left(\phi^{\dagger} \phi\right)^{2}+\frac{1}{2} \lambda_{2}\left(\xi^{\dagger} \xi\right)^{2} \\
& +\lambda_{3}\left(\phi^{\dagger} \phi\right)\left(\xi^{\dagger} \xi\right)+\lambda_{4} \phi^{T} \xi^{\dagger} \phi .
\end{aligned}
$$

We assume $\lambda_{4} \neq 0$, which manifests explicit lepton number violation and the mass of the triplet Higgs $M_{\xi} \sim \lambda_{4} \gg v$. The mass matrix of the scalars $\sqrt{2} \operatorname{Im} \phi^{0}$ and $\sqrt{2} \operatorname{Im} \xi^{0}$ is given by

$$
\mathcal{M}^{2}=\left(\begin{array}{cc}
-4 \lambda_{4} u & 2 \lambda_{4} v \\
2 \lambda_{4} v & -\lambda_{4} v^{2} / u
\end{array}\right),
$$

which tells us that one combination of these fields remains massless, which becomes the longitudinal mode of the $Z$ boson; while the other combination becomes massive with a mass of the order of triplet Higgs and hence the danger of $Z$ decaying into Majorons ${ }^{2}$ is absent in this model. The minimization of the scalar potential yields

$$
u=-\frac{\lambda_{4} v^{2}}{M_{\xi}^{2}},
$$

giving a seesaw mass to the left handed neutrinos

$$
m_{v i j}=f_{i j} u=-f_{i j} \frac{\lambda_{4} v^{2}}{M_{\xi}^{2}} .
$$

Note that in the left-right symmetric extension of the SM, which we will discuss in the next subsection, both type-I and type-II seesaw mechanisms are present together. The type-II seesaw mechanism can also provide a very attractive solution to leptogenesis, which we will discuss in the next section.

\subsection{Seesaw Mechanism: Type-III}

In type-III seesaw mechanism $[29,30]$ the SM is extended to include $S U(2)_{L}$ triplet fermions to realize the effective operator given in Equation (3) ${ }^{3}$. The Yukawa interactions in Equation (5) are generalized straightforwardly to $S U(2)_{L}$ triplet fermions $\Sigma$ with hypercharge $Y=0$. The corresponding interaction Lagrangian is given by

$$
-\mathcal{L}_{\text {type-III }}=h_{\Sigma i \alpha} \bar{\Psi}_{i L}\left(\vec{\Sigma}_{\alpha} \cdot \vec{\tau}\right) \tilde{\phi}+\frac{1}{2} M_{\Sigma \alpha \beta} \vec{\Sigma}_{\alpha}^{c T} C^{-1} \vec{\Sigma}_{\beta}^{c}+\text { h.c. },
$$

where $\alpha=1,2,3$. In exactly similar manner as in the case of type-I seesaw, one obtains for $M_{\Sigma} \gg v$, the left handed neutrino mass

$$
m_{v i j}=-v^{2} h_{\Sigma i \alpha} M_{\Sigma \beta \alpha}^{-1} h_{\Sigma j \beta}^{T}
$$

${ }^{2}$ Majorons correspond to Goldstone bosons associated with the spontaneous breaking of a global lepton number symmetry.

${ }^{3} \mathrm{Ma}$ [30] established the nomenclature Types I, II, III, for the three and only three tree-level seesaw mechanisms. 


\subsection{Radiative Models of Neutrino Mass}

Small neutrino masses can also be induced via radiative corrections. The advantage of these models is that without introducing a very large scale into the theory the smallness of the neutrino masses can be addressed. In fact, several of these models can explain naturally the smallness of the neutrino masses with only $\mathrm{TeV}$ scale new particles. Thus, new physics scale in these models can be as low as $\mathrm{TeV}$, which can be probed in current and next generation colliders.

One realization of this idea is the so-called Zee model [31, 32], where one extends the SM to have two (or more) Higgs doublets $\phi_{1}$ and $\phi_{2}$, and a scalar $\eta^{+}$which transforms under the SM gauge group $S U(3)_{c} \times S U(2)_{L} \times U(1)_{Y}$ as $(1,1,1)$. The lepton number violating Yukawa couplings are given by

$$
\mathcal{L}_{\mathrm{Zee}}=f_{i j} \psi_{i L}^{T} C^{-1} \psi_{j L} \eta^{+}+\mu \varepsilon_{a b} \phi_{a} \phi_{b} \eta^{-}+\text {h.c. }
$$

where $f_{i j}$ is antisymmetric in the family indices $i, j$ and $\varepsilon_{a b}$ is the totally antisymmetric tensor. Now, the VEV of the SM Higgs doublet allows mixing between the singlet charged scalar and the charged component of the second Higgs doublet, resulting in a neutrino mass induced through the one-loop diagram showed in Figure 1 (left). The antisymmetric couplings of $\eta^{+}$with the leptons make the diagonal terms of the mass matrix vanish, with the non-diagonal entries given by

$$
m_{i j}^{v}(i \neq j)=A f_{i j}\left(m_{i}^{2}-m_{j}^{2}\right)
$$

where $i, j=e, \mu, \tau$ and $A$ is a numerical constant. In the Zee model, if the second Higgs doublet is replaced by a doubly charged singlet scalar $\zeta^{++}$, then one gets what is called ZeeBabu Model [33, 34]. In this model a Majorana neutrino mass can be obtained through a two loop diagram shown in Figure 1(right). In fact, there are several other radiative models of Majorana neutrino mass such as the Ma model [35] connecting the Majorana neutrino mass to dark matter at oneloop; Krauss-Nasri-Trodden model [36] and Aoki-KanemuraSato model [37] giving neutrino mass at the three loop level with a dark matter candidate in the loop; Gustafsson-NoRivera model [38] involving a three loop diagram with a dark matter candidate and the $W$ boson; and KanemuraSugiyama model [39] utilizing an extension of the Higgs triplet model. There are also models for radiative Dirac neutrino masses such as the Nasri-Moussa model [40] utilizing a softly broken symmetry; Gu-Sarkar model [41] with dark matter candidates in the loop; Kanemura-Matsui-Sugiyama model [42] utilizing an extension of the two Higgs doublet model; BonillaMa-Peinado-Valle model where the Dirac neutrino masses are generated at two-loops with dark matter in the loop [43], etc.

\section{LEFT-RIGHT SYMMETRIC THEORIES}

The SM gauge group $\mathcal{G}_{\mathrm{SM}} \equiv S U(3)_{c} \times S U(2)_{L} \times U(1)_{Y}$ explains the $(V-A)$ structure of the weak interaction and parity violation, which is reflected by the trivial transformation of all right handed fields under $S U(2)_{L}$. However, the origin of parity violation is not explained within the SM, and it is natural to seek an explanation for parity violation starting from a parity conserved theory at some higher energy scale. This motivated a left-right symmetric extension of the SM gauge theory, called the LeftRight Symmetric Model (LRSM) [44-49], in which the Standard Model gauge group is extended to

$$
\mathcal{G}_{L R} \equiv S U(3)_{c} \times S U(2)_{L} \times S U(2)_{R} \times U(1)_{B-L}
$$

where $B-L$ is the difference between baryon (B) and lepton (L) numbers. The left-right symmetric theory, initially proposed to explain the origin of parity violation in low-energy weak interactions has come a long way answering various other issues like small neutrino mass, dark matter as left by the Standard Model. Originally suggested by Pati-Salam, the model has been studied over and over because of its versatility and many alternative formulations of the model have also been proposed. The model stands on the foundation of a complete symmetry between left and right which means Parity is an explicit symmetry in it until spontaneous symmetry breaking occurs. As evident from the gauge group, the natural inclusion of a righthanded neutrino in it makes the issue of neutrino mass an easy affair to discuss. Three new gauge bosons namely $W_{R}^{ \pm}$that are the heavier parity counterparts of $W_{L}^{ \pm}$of the standard model and a $Z^{\prime}$ boson analogous to the $Z$ boson also find place in the framework. LRSM breaks down to Standard Model gauge theory at low energy scales, $S U(2)_{L} \times S U(2)_{R} \times U(1)_{B-L} \times$ $S U(3)_{C} \longrightarrow S U(2)_{L} \times U(1)_{Y} \times S U(3)_{C}$. It has been noticed that the choices of Higgs and their mass scales in the model offers rich phenomenology which can be verified at the current and planned experiments.
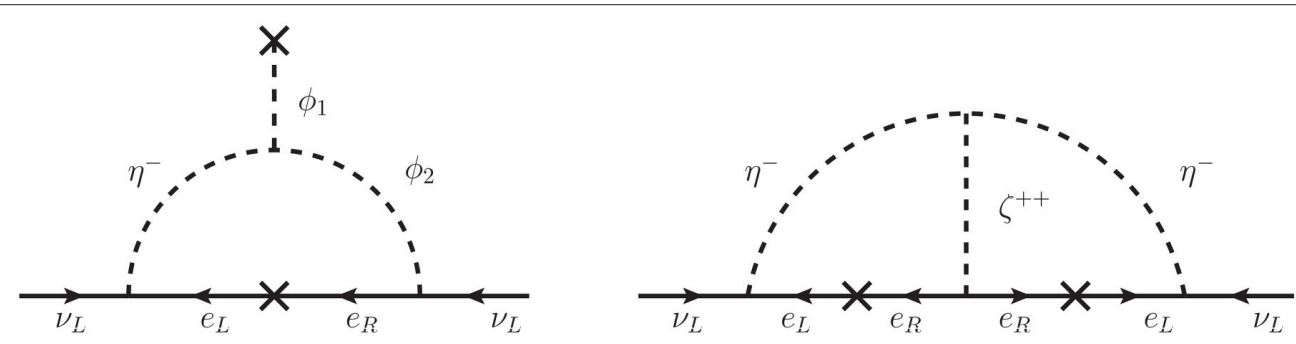

FIGURE 1 | (Left) one-loop diagram diagram generating neutrino mass in Zee model. (Right) two loop diagram generating neutrino mass in Zee-Babu model. 
The basic framework and properties of Left-Right Symmetric Models are already discussed at length in various original works [46-48], thus we only intend to study here various seesaw mechanisms for the generation of neutrino mass and its implications to leptogenesis in various Left-Right Symmetric models.

A very brief sketch of the manifest left-right symmetric model is given here. The model is based on the gauge group,

$$
\mathcal{G}_{L R} \equiv S U(2)_{L} \times S U(2)_{R} \times U(1)_{B-L} \times S U(3)_{C}
$$

The electric charge $Q$ is difined as,

$$
Q=T_{3 L}+T_{3 R}+\frac{B-L}{2}=T_{3 L}+Y
$$

Here, $T_{3 L}$ and $T_{3 R}$ are, respectively, the third components of isospin of the gauge groups $S U(2)_{L}$ and $S U(2)_{R}$, and $Y$ is the hypercharge. The particle spectrum of a generic LRSM can be sketched as,

$$
\begin{aligned}
& \ell_{L}=\left(\begin{array}{l}
v L \\
e_{L}
\end{array}\right) \sim(\mathbf{2}, \mathbf{1},-\mathbf{1}, \mathbf{1}), \ell_{R}=\left(\begin{array}{l}
v_{R} \\
e_{R}
\end{array}\right) \sim(\mathbf{1}, \mathbf{2},-\mathbf{1}, \mathbf{1}), \\
& q_{L}=\left(\begin{array}{l}
u_{R} \\
d_{R}
\end{array}\right) \sim\left(\mathbf{2}, \mathbf{1}, \frac{1}{3}, \mathbf{3}\right), \quad q_{R}=\left(\begin{array}{l}
u_{R} \\
d_{R}
\end{array}\right) \sim\left(\mathbf{1}, \mathbf{2}, \frac{1}{3}, \mathbf{3}\right) .
\end{aligned}
$$

The spontaneous symmetry breaking of the gauge group which occurs in two steps gives masses to fermions including neutrinos. In the first step the gauge group $S U(2)_{L} \times S U(2)_{R} \times$ $U(1)_{B-L} \times S U(3)_{C}$ breaks down to $S U(2)_{L} \times U(1)_{Y} \times$ $S U(3)_{C}$ i.e., the $\mathrm{SM}$ gauge group. This gauge group then breaks down to $U(1)_{e m} \times S U(3)_{C}$. However these symmetry breakings totally depend upon the choices of Higgs that we consider in the framework and their mass scales. Thus, in this review we intend to discuss fermion masses emphasizing on neutrino mass in possible choices of symmetry breakings of LRSM.

\subsection{LRSM With Bidoublet $(B-L=0)$ and Doublets $(B-L=-1)$.}

Here we use Higgs bidoublet $\Phi$ to implement the symmetry breaking of SM down to low energy theory leading to charged fermion masses. The symmetry breaking of LRSM to SM occurs via $\mathrm{RH}$ Higgs doublet $H_{R}(B-L=-1)$. We need the lefthanded counterpart $H_{L}$ to ensure left-right invariance. The fermions including usual quarks and leptons along with scalars are presented in Table 1.

The matrix structure of the scalar fields looks as follows,

$$
\begin{aligned}
\Phi & \equiv\left(\begin{array}{cc}
\phi_{1}^{0} & \phi_{2}^{+} \\
\phi_{1}^{-} & \phi_{2}^{0}
\end{array}\right) \sim(\mathbf{2}, \mathbf{2}, \mathbf{0}, \mathbf{1}), \\
H_{L} & \equiv\left(\begin{array}{c}
h_{L}^{+} \\
h_{L}^{0}
\end{array}\right) \sim(\mathbf{2}, \mathbf{1},-\mathbf{1}, \mathbf{1}), \quad H_{R} \equiv\left(\begin{array}{c}
h_{R}^{+} \\
h_{R}^{0}
\end{array}\right) \sim(\mathbf{1}, \mathbf{2},-\mathbf{1}, \mathbf{1}) .
\end{aligned}
$$

TABLE 1 | LRSM representations of extended field content.

\begin{tabular}{cccccc}
\hline & Fields & $\boldsymbol{S U}_{(2)_{\mathbf{L}}}$ & $\boldsymbol{S U}_{(2)_{\boldsymbol{R}}}$ & $\boldsymbol{B}-\mathbf{L}$ & $\boldsymbol{S U}(\mathbf{3})_{\boldsymbol{C}}$ \\
\hline Fermions & $q_{\boldsymbol{L}}$ & 2 & 1 & $1 / 3$ & 3 \\
& $q_{R}$ & 1 & 2 & $1 / 3$ & 3 \\
& $\ell_{L}$ & 2 & 1 & -1 & 1 \\
& $\ell_{R}$ & 1 & 2 & -1 & 1 \\
\hline \multirow{6}{*}{ Scalars } & $\Phi$ & 2 & 2 & 0 & 1 \\
& $H_{L}$ & 2 & 1 & -1 & 1 \\
& $H_{R}$ & 1 & 2 & -1 & 1 \\
\hline
\end{tabular}

With usual quarks and leptons the Yukawa Lagrangian reads as,

$$
-\mathcal{L}_{Y u k} \supset \overline{q_{L}}\left[Y_{1} \Phi+Y_{2} \tilde{\Phi}\right] q_{R}+\overline{\ell_{L}}\left[Y_{3} \Phi+Y_{4} \tilde{\Phi}\right] \ell_{R}+\text { h.c. }
$$

where $\widetilde{\Phi}=\sigma_{2} \Phi^{*} \sigma_{2}$ and $\sigma_{2}$ is the second Pauli matrix. When the scalar bidoublet $(\Phi)$ takes non-zero VEV ,

$$
\langle\Phi\rangle=\left(\begin{array}{cc}
v 1 & 0 \\
0 & v 2
\end{array}\right)
$$

it gives masses to quarks and charged leptons in the following manner,

$$
\begin{aligned}
& M_{u}=Y_{1} v 1+Y_{2} v_{2}^{*}, \quad M_{d}=Y_{1} v 2+Y_{2} v_{1}^{*}, \\
& M_{e}=Y_{3} v 2+Y_{4} v_{1}^{*} .
\end{aligned}
$$

It also yields Dirac mass for light neutrinos as

$$
M_{D}^{v} \equiv M_{D}=Y_{3} v_{1}+Y_{4} v_{2}^{*}
$$

The only role that the Higgs doublets play here is helping in the spontaneous symmetry breaking of LRSM to SM. It is also important to note that the breaking of $S U(2)_{R}$ by doublet Higgs leads to Dirac neutrinos.

\subsection{LRSM With Bidoublet $(B-L=0)$ and Triplets $(B-L=2)$.}

Along with the bidoublet $\Phi$, here we use triplets $\Delta_{L}, \Delta_{R}$ for the spontaneous symmetry breakings.

$$
\begin{aligned}
\Phi & \equiv\left(\begin{array}{cc}
\phi_{1}^{0} & \phi_{2}^{+} \\
\phi_{1}^{-} & \phi_{2}^{0}
\end{array}\right) \sim(\mathbf{2}, \mathbf{2}, \mathbf{0}, \mathbf{1}), \\
\Delta_{L} & \equiv\left(\begin{array}{cc}
\delta_{L}^{+} / \sqrt{2} & \delta_{L}^{++} \\
\delta_{L}^{0} & -\delta_{L}^{+} / \sqrt{2}
\end{array}\right) \sim(\mathbf{3}, \mathbf{1}, \mathbf{2}, \mathbf{1}), \\
\Delta_{R} & \equiv\left(\begin{array}{cc}
\delta_{R}^{+} / \sqrt{2} & \delta_{R}^{++} \\
\delta_{R}^{0} & -\delta_{R}^{+} / \sqrt{2}
\end{array}\right) \sim(\mathbf{1}, \mathbf{3}, \mathbf{2}, \mathbf{1}),
\end{aligned}
$$

The particle content of the model is shown in Table 2.

The Yukawa Lagrangian is given by

$$
\begin{aligned}
-\mathcal{L}_{Y u k} \supset & \overline{q_{L}}\left[Y_{1} \Phi+Y_{2} \widetilde{\Phi}\right] q_{R}+\overline{\ell_{L}}\left[Y_{3} \Phi+Y_{4} \widetilde{\Phi}\right] \ell_{R} \\
& +f\left[\overline{\left(\ell_{L}\right)^{c}} \ell_{L} \Delta_{L}+\overline{\left(\ell_{R}\right)^{c}} \ell_{R} \Delta_{R}\right]+\text { h.c. }
\end{aligned}
$$


TABLE 2 | LRSM representations of extended field content.

\begin{tabular}{llcccc}
\hline & Fields & $\boldsymbol{S U}(\mathbf{2})_{\boldsymbol{L}}$ & $\mathbf{S U}(\mathbf{2})_{\boldsymbol{R}}$ & $\boldsymbol{B}-\boldsymbol{L}$ & $\mathbf{S U}(\mathbf{3})_{\boldsymbol{C}}$ \\
\hline \multirow{3}{*}{ Fermions } & $q_{L}$ & 2 & 1 & $1 / 3$ & 3 \\
& $q_{R}$ & 1 & 2 & $1 / 3$ & 3 \\
& $\ell_{L}$ & 2 & 1 & -1 & 1 \\
& $\ell_{R}$ & 1 & 2 & -1 & 1 \\
\hline \multirow{3}{*}{ Scalars } & $\Phi$ & 2 & 2 & 0 & 1 \\
& $\Delta_{L}$ & 3 & 1 & 2 & 1 \\
& $\Delta_{R}$ & 1 & 3 & 2 & 1
\end{tabular}

The scalar triplets $\Delta_{L}, \Delta_{R}$ give Majorana masses to light lefthanded and heavy right-handed neutrinos. The neutral lepton mass matrix is given by

$$
M_{v}=\left(\begin{array}{ll}
M_{L} & M_{D} \\
M_{D}^{T} & M_{R}
\end{array}\right),
$$

Here $M_{L}=f_{L}\left\langle\Delta_{L}\right\rangle=f v_{L}\left(M_{R}=f_{R}\left\langle\Delta_{R}\right\rangle=f v_{R}\right)$ denoted as the Majorana mass matrix for left-handed (right-handed) neutrinos and $M_{D}=Y_{3} v_{1}+Y_{4} v_{2}$ is the Dirac neutrino mass matrix connecting light-heavy neutrinos. The complete diagonalization results type-I+II seesaw formula for light neutrinos as,

$$
m_{v}=M_{L}-m_{D} M_{R}^{-1} m_{D}^{T}=m_{v}^{I I}+m_{v}^{I}
$$

\subsection{LRSM With Inverse Seesaw}

In canonical seesaw mechanisms, the tiny mass of light neutrinos is explained with large value of seesaw scale thereby making it inaccessible to the ongoing collider experiments. On the other hand, the light neutrino masses may arise from low scale seesaw mechanisms like inverse seesaw $[50,51]$ where the seesaw scale can be probed at upcoming accelerators. The inverse seesaw mechanism in LRSM can be realized with the following particle content;

\section{Fermions :}

$$
\begin{aligned}
& q_{L}=\left(\begin{array}{l}
u_{L} \\
d_{L}
\end{array}\right) \sim(2,1,1 / 3,3), q_{R}=\left(\begin{array}{l}
u_{R} \\
d_{R}
\end{array}\right) \sim(1,2,1 / 3,3), \\
& \ell_{L}=\left(\begin{array}{l}
v_{L} \\
e_{L}
\end{array}\right) \sim(2,1,-1,1), \ell_{R}=\left(\begin{array}{l}
v_{R} \\
e_{R}
\end{array}\right) \sim(1,2,-1,1), \\
& S \sim(1,1,1,0),
\end{aligned}
$$

Scalars :

$$
\begin{aligned}
& H_{L}=\left(\begin{array}{l}
h_{L}^{+} \\
h_{L}^{0}
\end{array}\right) \sim(\mathbf{2}, \mathbf{1}, \mathbf{1}, \mathbf{1}), \quad H_{R}=\left(\begin{array}{l}
h_{R}^{+} \\
h_{R}^{0}
\end{array}\right) \sim(\mathbf{1}, \mathbf{2}, \mathbf{1}, \mathbf{1}) \\
& \Phi=\left(\begin{array}{cc}
\phi_{1}^{0} & \phi_{2}^{+} \\
\phi_{1}^{-} & \phi_{2}^{0}
\end{array}\right) \sim(\mathbf{2}, \mathbf{2}, \mathbf{0}, \mathbf{1}),
\end{aligned}
$$

The fermion sector here comprises of the usual quarks and leptons plus one extra fermion singlet per generation. The scalar sector holds the doublets $H_{L, R}$ with $B-L$ charge -1 and the bidoublet $\Phi$ with $B-L$ charge 0 . The Yukawa Langrangian for inverse seesaw mechanism is given by,

$$
\begin{aligned}
-\mathcal{L}_{Y u k} & =\overline{\ell_{L}}\left[Y_{3} \Phi+Y_{4} \widetilde{\Phi}\right] \ell_{R}+F \overline{\left(\ell_{R}\right)} H_{R} S_{L}^{c}+\mu \overline{S_{L}^{c}} S_{L}+\text { h.c. } \\
& \supset M_{D} \overline{\nu_{L}} N_{R}+M \overline{N_{R}} S_{L}+\mu_{S} \overline{S_{L}^{c}} S_{L}+\text { h.c. }
\end{aligned}
$$

After spontaneous symmetry breaking the resulting neutral lepton mass matrix reads as follows,

$$
\mathbb{M}=\left(\begin{array}{ccc}
0 & M_{D} & 0 \\
M_{D}^{T} & 0 & M \\
0 & M^{T} & \mu_{S}
\end{array}\right) .
$$

With the mass hierarchhy $m_{D}, M \gg \mu_{S}$, the light neutrino mass formula is given by,

$$
m_{v}=M_{D}\left(M^{T}\right)^{-1} \mu M^{-1} M_{D}^{T} .
$$

\subsection{LRSM With Linear Seesaw}

Another interesting low scale seesaw type is linear seesaw mechanism $[52,53]$ which can be realized with the following particle content in a LRSM.

$$
\begin{aligned}
& \text { Fermions: } \\
& Q_{L}=\left(\begin{array}{l}
u_{L} \\
d_{L}
\end{array}\right) \sim(2,1,1 / 3,3), Q_{R}=\left(\begin{array}{l}
u_{R} \\
d_{R}
\end{array}\right) \sim(1,2,1 / 3,3), \\
& \ell_{L}=\left(\begin{array}{l}
v_{L} \\
e_{L}
\end{array}\right) \sim(2,1,-1,1), \ell_{R}=\left(\begin{array}{c}
v_{R} \\
e_{R}
\end{array}\right) \sim(1,2,-1,1), \\
& S \sim(\mathbf{1}, \mathbf{1}, \mathbf{0}, \mathbf{1}),
\end{aligned}
$$

Scalars :

$$
\begin{aligned}
& H_{L}=\left(\begin{array}{c}
h_{L}^{+} \\
h_{L}^{0}
\end{array}\right) \sim(\mathbf{2}, \mathbf{1}, \mathbf{1}, \mathbf{1}) \quad H_{R}=\left(\begin{array}{l}
h_{R}^{+} \\
h_{R}^{0}
\end{array}\right) \sim(\mathbf{1}, \mathbf{2}, \mathbf{1}, \mathbf{1}) \\
& \Phi=\left(\begin{array}{cc}
\phi_{1}^{0} & \phi_{2}^{+} \\
\phi_{1}^{-} & \phi_{2}^{0}
\end{array}\right) \sim(\mathbf{2}, \mathbf{2}, \mathbf{0}, \mathbf{1}) .
\end{aligned}
$$

The scalars take non-zero vev as follows:

$$
\langle\Phi\rangle=k_{1}, k_{2}, \quad\left\langle H_{L}\right\rangle=v_{L}, \quad\left\langle H_{R}\right\rangle=v_{R}
$$

Let us write down the relevant Yukawa terms in the Lagrangian that contribute to the fermion masses:

$$
\begin{aligned}
\mathcal{L}_{\text {Yuk }}= & h_{\ell} \overline{\ell_{R}} \Phi \ell_{L}+\widetilde{h_{\ell}} \overline{\ell_{R}} \widetilde{\Phi} \ell_{L}+f_{R} \bar{S} \widetilde{H_{R}} \ell_{R}+f_{L} \bar{S} \widetilde{H_{L}} \ell_{L} \\
& +\mu_{S} \overline{\left(S_{L}\right)^{c}} S_{L}+\text { h.c., }
\end{aligned}
$$

where $\widetilde{H}_{j}=i \tau_{2} H_{j}^{*}$ with $j=L, R$ and $\widetilde{\Phi}=\tau_{2} \Phi^{*} \tau_{2}$. The singlet Majorana field $S$ in Equation (34) is defined as

$$
S=\frac{S_{L}+\left(S_{L}\right)^{c}}{\sqrt{2}} .
$$


resulting in the neutral lepton mass matrix

$$
\begin{aligned}
M_{N} & =\left(\begin{array}{ccc}
0 & h_{\ell} k_{1}+\tilde{h_{\ell}} k_{2} & f_{L} v_{L} \\
h_{\ell}^{T} k_{1}+\tilde{h}_{\ell}^{T} k_{2} & 0 & f_{R} v_{R} \\
f_{L}^{T} v_{L} & f_{R}^{T} v_{R} & \mu_{S}
\end{array}\right) \\
& \equiv\left(\begin{array}{ccc}
0 & m_{D} & m_{L} \\
m_{D}^{T} & 0 & M \\
m_{L}^{T} & M^{T} & \mu_{S}
\end{array}\right) .
\end{aligned}
$$

The violation of lepton number by two units arises here through the combination $m_{L}$ and $\mu_{S}$. As a result, assuming $m_{L} \ll m_{D}<M$, one gets the light Majorana masses of the active neutrinos to be

$$
\begin{aligned}
m_{v} & =\left[m_{D} M^{T^{-1}} m_{L}^{T}+m_{L} M^{-1} m_{D}^{T}\right] \\
& =\left[m_{D}\left(f_{L} f_{R}^{-1}\right)^{T}+\left(f_{L} f_{R}^{-1}\right) m_{D}^{T}\right] \times \frac{\left(\mu_{1} k_{1}+\mu_{2} k_{2}\right)}{M^{\prime} \eta_{P}} .
\end{aligned}
$$

The last line in Equation (37) follows from the fact that in leftright symmetric model where Parity and $S U(2)_{R}$ breaking occurs at different scales $v_{L}$ is given by

$$
v_{L} \simeq-\frac{\left(\mu_{1} k_{1}+\mu_{2} k_{2}\right) v_{R}}{M^{\prime} \eta_{P}}
$$

where $\mu_{1}, \mu_{2}$ are the trilinear terms arising in the Higgs potential involving Higgs bidoublet and Higgs doublets, $\eta_{P}$ is the parity breaking scale and $M^{\prime}$ is the $S U(2)_{R}$ breaking scale. From Equation (37) it is clear that the light neutrino mass is suppressed by the parity breaking scale $\eta_{P} \simeq M^{\prime}$. The $f_{L}$ and $f_{R}$ are Majorana couplings, $k_{1}, k_{2}$ being VEV of Higgs bidoublet while $v_{L}\left(v_{R}\right)$ is the VEV of LH (RH) scalar doublet. The smallness of $v_{L}$ thus ensures the smallness of the observed sub-eV scale neutrino masses. The $S U(2)_{R} \times U(1)_{B-L}$ breaking scale $v_{R}$ can be as low as a few $\mathrm{TeV}$. This is in contrast to the usual left-right symmetric model without $D$-parity, where the neutrino mass is suppressed by $v_{R}$ and hence cannot be brought to TeV scales easily [54].

In addition we get two heavy pseudo-Dirac states, whose masses are separated by the light neutrino mass, given by

$$
\widetilde{M} \approx \pm M+m_{v}
$$

In the above equation, the small masses of active neutrinos can arise through small values of $m_{L} / M$. As a result of $M$ around $\mathrm{TeV}$ and $m_{D}$ in the range of $100 \mathrm{GeV}$, sizable mixing between the light and heavy states arises, and the Pseudo-Dirac pair with mass $M$ can be probed at colliders ${ }^{4}$.

\subsection{LRSM With Extended Seesaw}

The LRSM here is extended with the addition of a neutral fermion $S_{L}$ per generation to the usual quarks and leptons. ${ }^{5}$ The scalar sector consists of bidoublet $\Phi$ with $B-L=0$, triplets $\Delta_{L} \oplus \Delta_{R}$

\footnotetext{
${ }^{4}$ Most often the linear seesaw is assumed to be realized with $M \gg m_{D} \sim m_{L} \sim$ $100 \mathrm{GeV}$, which results in the same expression for the $m_{\nu}$. This would result in unobservable heavy fermions and negligible mixing.

${ }^{5}$ The discussion of extended seesaw mechanism can be found in Gavela et al. [55], Barry et al. [56], Zhang [57] and Dev and Pilaftsis[58].
}

with $B-L=2$ and doublets $H_{L} \oplus H_{R}$ with $B-L=-1$. We call the model Extended LR model and thus the seesaw mechanism is called extended seesaw. Table 3 shows the complete particle spectrum.

The leptonic Yukawa interaction terms can be written as,

$$
\begin{aligned}
-\mathcal{L}_{Y u k}= & \overline{\ell_{L}}\left[Y_{3} \Phi+Y_{4} \widetilde{\Phi}\right] \ell_{R}+f\left[\overline{\left(\ell_{L}\right)^{c}} \ell_{L} \Delta_{L}+\overline{\left(\ell_{R}\right)^{c}} \ell_{R} \Delta_{R}\right] \\
& +F \overline{\left(\ell_{R}\right)} H_{R} S_{L}^{c}+F^{\prime} \overline{\left(\ell_{L}\right)} H_{L} S_{L}+\mu_{S} \overline{S_{L}^{c}} S_{L}+\text { h.c. . } \\
\supset & M_{D} \overline{v_{L}} N_{R}+M_{L} \overline{v_{L}^{c}} v_{L}+M_{R} \overline{N_{R}^{c}} N_{R} \\
& +M \overline{N_{R}} S_{L}+\mu_{L} \overline{v_{L}^{c}} S_{L}+\mu_{S} \overline{S_{L}^{c}} S_{L}
\end{aligned}
$$

The neutral lepton mass matrix comes out to be;

$$
\mathbb{M}_{\nu}=\left(\begin{array}{ccc}
M_{L} & M_{D} & \mu_{L} \\
M_{D}^{T} & M_{R} & M \\
\mu_{L}^{T} & M^{T} & \mu_{S}
\end{array}\right)
$$

in the basis $\left(v_{L}, N_{R}^{c}, S_{L}\right)$ after spontaneous symmetry breaking. The individual elements of the matrix hold the following meaning; $M_{D}=Y\langle\Phi\rangle$ measures the light-heavy neutrino mixing and is usually called the Dirac neutrino mass matrix, $M_{N}=$ $f v_{R}=f\left\langle\Delta_{R}\right\rangle\left(M_{L}=f v_{L}=f\left\langle\Delta_{L}\right\rangle\right)$ is the Majorana mass term for heavy (light) neutrinos, $M=F\left\langle H_{R}\right\rangle$ is the $N-S$ mixing matrix, $\mu_{L}=F^{\prime}\left\langle H_{L}\right\rangle$ stands for the small mass term connecting $v-S$ and $\mu_{S}$ is the Majorana mass term for the singlet fermion $S_{L}$. Inverse Seesaw:- In Equation (42), following the mass hierarchy $M \gg M_{D} \gg \mu_{S}$ and with the assumption that $M_{L}, M_{R}, \mu_{L} \rightarrow 0$ one obtains the inverse seesaw mass formula for light neutrinos [59]

$$
m_{\nu}=\left(\frac{M_{D}}{M}\right) \mu_{s}\left(\frac{M_{D}}{M}\right)^{T}
$$

Let us have a look at the model parameters of inverse seesaw framework and see how the light neutrino mass can be parametrized in terms of these.

$$
\left(\frac{m_{v}}{0.1 \mathrm{eV}}\right)=\left(\frac{M_{D}}{100 \mathrm{GeV}}\right)^{2}\left(\frac{\mu_{s}}{\mathrm{keV}}\right)\left(\frac{M}{10^{4} \mathrm{GeV}}\right)^{-2}
$$

TABLE 3 | LRSM representations of extended field content.

\begin{tabular}{cccccc}
\hline \multirow{3}{*}{ Fermions } & Fields & $\boldsymbol{S U}(\mathbf{2})_{\boldsymbol{L}}$ & $\boldsymbol{S U}(\mathbf{2})_{\boldsymbol{R}}$ & $\boldsymbol{B}-\boldsymbol{L}$ & $\boldsymbol{S U}(\mathbf{3})_{\boldsymbol{C}}$ \\
& $q_{\boldsymbol{L}}$ & 2 & 1 & $1 / 3$ & 3 \\
& $q_{R}$ & 1 & 2 & $1 / 3$ & 3 \\
& $\ell_{L}$ & 2 & 1 & -1 & 1 \\
& $\ell_{R}$ & 1 & 2 & -1 & 1 \\
& $S_{L}$ & 1 & 1 & 0 & 1 \\
& $S^{*}$ & 2 & 2 & 0 & 1 \\
& $H_{L}$ & 2 & 1 & -1 & 1 \\
& $H_{R}$ & 1 & 2 & -1 & 1 \\
& $\Delta_{L}$ & 3 & 1 & 2 & 1
\end{tabular}


Testable collider phenomenology can be expected in such a scenario because $M$ lies at a few TeV scale which allows large leftright mixing. For an extension of such a scenario which allows large LNV and LFV one may refer the work [60].

Linear Seesaw:- Alternatively, in Equation (42), the assumption of $M_{L}, M_{R}, \mu_{S} \rightarrow 0$ leads to the linear seesaw mass formula for light neutrinos given by Deppisch et al. [61]

$$
m_{v}=M_{D}^{T} M^{-1} \mu_{L}+\text { transpose },
$$

whereas the heavy neutrinos form a pair of pseudo-Dirac states with masses

$$
M_{ \pm} \approx \pm M+m_{v}
$$

Type-II Seesaw Dominance:- On the other hand a type-II seesaw dominance can be realized with the assumption that $\mu_{L}, \mu_{S} \rightarrow 0$ in Equation (42). This allows large left-right mixing and thus leads to an interesting scenario.

A natural type-II seesaw dominance can be realized from the following Yukawa interactions

$$
\begin{aligned}
-\mathcal{L}_{Y u k}= & \overline{\ell_{L}}\left[Y_{3} \Phi+Y_{4} \widetilde{\Phi}\right] \ell_{R}+f\left[\overline{\left(\ell_{L}\right)^{c}} \ell_{L} \Delta_{L}+\overline{\left(\ell_{R}\right)^{c}} \ell_{R} \Delta_{R}\right] \\
& +F \overline{\left(\ell_{R}\right)} H_{R} S_{L}^{c}+\text { h.c. } \\
\supset & M_{D} \overline{\nu_{L}} N_{R}+M_{L} \overline{v_{L}^{c}} \nu_{L}+M_{R} \overline{N_{R}^{c}} N_{R}+M \overline{N_{R}} S_{L}+\text { h.c. }
\end{aligned}
$$

The gauge singlet mass term $\mu_{S} \overline{S c} S$ does not appear in the above Lagrangian since we have considered this to be zero or negligbly small to suppress the generic inverse seesaw contribution involving $\mu_{S}$. We have also assumed the induced VEV for $H_{L}$ to be zero, i.e., $\left\langle H_{L}\right\rangle \rightarrow 0$.

Now the complete $9 \times 9$ mass matrix for the neutral fermions in flavor basis can be written as

$$
\mathbb{M}=\left(\begin{array}{c|ccc} 
& v_{L} & S_{L} & N_{R}^{c} \\
\hline v_{L} & M_{L} & 0 & M_{D} \\
S_{L} & 0 & 0 & M \\
N_{R}^{c} & M_{D}^{T} & M^{T} & M_{R}
\end{array}\right)
$$

The heaviest right-handed neutrinos can be integrated out following the standard formalism of seesaw mechanism. Using mass hierarchy $M_{R}>M>M_{D} \gg M_{L}$ one obtains

$$
\begin{aligned}
\mathbb{M}^{\prime} & =\left(\begin{array}{cc}
M_{L} & 0 \\
0 & 0
\end{array}\right)-\left(\begin{array}{c}
M_{D} \\
M
\end{array}\right) M_{R}^{-1}\left(M_{D}^{T} M^{T}\right) \\
& =\left(\begin{array}{cc}
M_{L}-M_{D} M_{R}^{-1} M_{D}^{T} & -M_{D} M_{R}^{-1} M^{T} \\
M M_{R}^{-1} M_{D}^{T} & -M M_{R}^{-1} M^{T}
\end{array}\right),
\end{aligned}
$$

where the intermediate block diagonalised neutrino states are modified as

$$
\begin{aligned}
& v^{\prime}=v_{L}-M_{D} M_{R}^{-1} N_{R}^{c}, \\
& S^{\prime}=S_{L}-M_{D} M_{R}^{-1} N_{R}^{c}, \\
& N^{\prime}=N_{R}^{c}+\left(M_{R}^{-1} M_{D}^{T}\right)^{*} v_{L}+\left(M_{R}^{-1} M^{T}\right)^{*} S_{L} .
\end{aligned}
$$

The following transformation relates the intermediate block diagonalised neutrino states to the flavor eigenstates.

$$
\left(\begin{array}{c}
v^{\prime} \\
S^{\prime} \\
N^{\prime}
\end{array}\right)=\left(\begin{array}{ccc}
\mathbb{I} & \mathbb{O} & -M_{D} M_{R}^{-1} \\
\mathbb{O} & \mathbb{I} & -M M_{R}^{-1} \\
\left(M_{D} M_{R}^{-1}\right)^{\dagger} & \left(M M_{R}^{-1}\right)^{\dagger} & \mathbb{I}
\end{array}\right)\left(\begin{array}{c}
v_{L} \\
S_{L} \\
N_{R}^{c}
\end{array}\right)
$$

In the mass matrix $\mathbb{M}^{\prime}$ the $(2,2)$ entry is larger than other entries in the limit $M_{R}>M>M_{D} \gg M_{L}$. The same procedure can be repeated in Equation (48) and $S^{\prime}$ can be integrated out. Now the mass formula for light neutrino is given by

$$
\begin{aligned}
m_{v}= & {\left[M_{L}-M_{D} M_{R}^{-1} M_{D}^{T}\right] } \\
& -\left(-M_{D} M_{R}^{-1} M^{T}\right)\left(-M M_{R}^{-1} M^{T}\right)^{-1}\left(-M M_{R}^{-1} M_{D}^{T}\right) \\
= & {\left[M_{L}-M_{D} M_{R}^{-1} M_{D}^{T}\right]+M_{D} M_{R}^{-1} M_{D}^{T} } \\
= & M_{L}=m_{v}^{\mathrm{II}},
\end{aligned}
$$

and the physical block diagonalised states are

$$
\begin{aligned}
& \hat{v}=v_{L}-M_{D} M^{-1} S_{L} \\
& \hat{S}=S_{L}-M M_{R}^{-1} N_{R}^{c}+\left(M_{D} M^{-1}\right)^{\dagger} S_{L}
\end{aligned}
$$

with the corresponding block diagonalised transformation as

$$
\left(\begin{array}{l}
\hat{v} \\
\hat{S}
\end{array}\right)=\left(\begin{array}{cc}
\mathbb{I} & -M_{D} M^{-1} \\
\left(M M^{-1}\right)^{\dagger} & \mathbb{I}
\end{array}\right)\left(\begin{array}{l}
v^{\prime} \\
S^{\prime}
\end{array}\right)
$$

Following this block diagonalization procedure the flavor eigenstates can be related to mass eigenstates through the following transformation

$$
\left(\begin{array}{c}
v_{L} \\
S_{L} \\
N_{R}^{c}
\end{array}\right)=\left(\begin{array}{ccc}
\mathbb{I} & M_{D} M^{-1} & M_{D} M_{R}^{-1} \\
\left(M_{D} M^{-1}\right)^{\dagger} & \mathbb{I} & M M_{R}^{-1} \\
\mathbb{O} & -\left(M M_{R}^{-1}\right)^{\dagger} & \mathbb{I}
\end{array}\right)\left(\begin{array}{c}
v^{\prime} \\
S^{\prime} \\
N^{\prime}
\end{array}\right)
$$

Finally, the physical masses can be obtained by diagonalising the final block diagonalised mass matrices by a $9 \times 9$ unitary matrix $\mathrm{V}_{9 \times 9}$. The block diagonalised neutrino states can be expressed in terms of mass eigenstates as follows,

$$
\hat{v}_{\alpha}=U_{\nu \alpha i} v_{i}, \quad \hat{S}_{\alpha}=U_{S \alpha i} S_{i}, \quad \hat{N}_{\alpha}=U_{N \alpha i} N_{i} .
$$

while the block diagonalised mass matrices for light lefthanded neutrinos, heavy right-handed neutrinos and extra sterile neutrinos are

$$
\begin{aligned}
m_{v} & =M_{L}, \\
M_{N} & \equiv M_{R}=\frac{v_{R}}{v_{L}} M_{L}, \\
M_{S} & =-M M_{R}^{-1} M^{T} .
\end{aligned}
$$

Further these mass matrices can be diagonalised by respective $3 \times 3$ unitarity matrices as,

$$
\begin{aligned}
& m_{v}^{\text {diag }}=U_{v}^{\dagger} m_{v} U_{v}^{*}=\text { diag. }\left\{m_{1}, m_{2}, m_{3}\right\}, \\
& M_{S}^{\text {diag }}=U_{S}^{\dagger} M_{S} U_{S}^{*}=\operatorname{diag} .\left\{M_{S_{1}}, M_{S_{2}}, M_{S_{3}}\right\}, \\
& M_{N}^{\text {diag }}=U_{N}^{\dagger} M_{N} U_{N}^{*}=\operatorname{diag} .\left\{M_{N_{1}}, M_{N_{2}}, M_{N_{3}}\right\} .
\end{aligned}
$$


The complete block diagonalization results,

$$
\begin{aligned}
\widehat{\mathbb{M}} & =\mathrm{V}_{9 \times 9}^{\dagger} \mathbb{M} V_{9 \times 9}^{*}=(\mathbb{W} \cdot \mathbb{U})^{\dagger} \mathbb{M}(\mathbb{W} \cdot \mathbb{U}) \\
& =\operatorname{diag} .\left\{m_{1}, m_{2}, m_{3} ; M_{S_{1}}, M_{S_{2}}, M_{S_{3}} ; M_{N_{1}}, M_{N_{2}}, M_{N_{3}}\right\}
\end{aligned}
$$

where $\mathbb{W}$ is the block diagonalised mixing matrix and $\mathbb{U}$ is the unitarity matrix given by,

$$
\begin{aligned}
\mathbb{W} & =\left(\begin{array}{ccc}
\mathbb{I} & M_{D} M^{-1} & M_{D} M_{R}^{-1} \\
\left(M_{D} M^{-1}\right)^{\dagger} & \mathbb{I} & M M_{R}^{-1} \\
\mathbb{O} & -\left(M M_{R}^{-1}\right)^{\dagger} & \mathbb{I}
\end{array}\right), \\
\mathbb{U} & =\left(\begin{array}{ccc}
U_{v} & \mathbb{O} & \mathbb{O} \\
\mathbb{O} & U_{S} & \mathbb{O} \\
\mathbb{O} & \mathbb{O} & U_{N}
\end{array}\right) .
\end{aligned}
$$

Thus, the complete $9 \times 9$ unitary mixing matrix diagonalizing the neutral leptons is as follows

$$
\mathrm{V}=\mathbb{W} \cdot \mathbb{U}=\left(\begin{array}{ccc}
U_{v} & M_{D} M^{-1} U_{S} & M_{D} M_{R}^{-1} U_{N} \\
\left(M_{D} M^{-1}\right)^{\dagger} U_{v} & U_{S} & M M_{R}^{-1} U_{N} \\
\mathbb{O} & -\left(M M_{R}^{-1}\right)^{\dagger} U_{S} & U_{N}
\end{array}\right)
$$

Expressing Masses and Mixing in terms of $U_{\text {PMNS }}$ and light neutrino masses:- Usually, the light neutrino mass matrix is diagonalised by the $U_{\text {PMNS }}$ mixing matrix in the basis where the charged leptons are already diagonal i.e., $m_{v}^{\text {diag }}=$ $U_{\mathrm{PMNS}}^{\dagger} m_{\nu} U_{\mathrm{PMNS}}^{*}$. The structure of the Dirac neutrino mass matrix $M_{D}$ which is a complex matrix in general can be considered to be the up-quark type in LRSM. Its origin can be motivated from a high scale Pati-Salam symmetry or $\mathrm{SO}(10)$ GUT. If we consider $M$ to be diagonal and degenerate i.e., $M=$ $m_{S} \operatorname{diag}\{1,1,1\}$, then the mass formulas for neutral leptons are given by

$$
\begin{aligned}
& m_{v}=M_{L}=f v_{L}=U_{\mathrm{PMNS}} m_{v}^{\mathrm{diag}} U_{\mathrm{PMNS}}^{T}, \\
& M_{N} \equiv M_{R}=f v_{R}=\frac{v_{R}}{v_{L}} M_{L}=\frac{v_{R}}{v_{L}} U_{\mathrm{PMNS}} m_{v}^{\mathrm{diag}} U_{\mathrm{PMNS}}^{T}, \\
& M_{S}=-M M_{R}^{-1} M^{T}=-m_{S}^{2}\left[\frac{v_{R}}{v_{L}} U_{\mathrm{PMNS}} m_{v}^{\mathrm{diag}} U_{\mathrm{PMNS}}^{T}\right]^{-1},
\end{aligned}
$$

After some simplification the active $\mathrm{LH}$ neutrinos $v_{L}$, active $\mathrm{RH}$ neutrinos $N_{R}$ and heavy sterile neutrinos $S_{L}$ in the flavor basis are related to their mass basis as

$$
\begin{aligned}
& \left(\begin{array}{c}
v_{L} \\
S_{L} \\
N_{R}^{c}
\end{array}\right)_{\alpha}=\left(\begin{array}{ccc}
\mathrm{V}^{v v} & \mathrm{~V}^{v S} & \mathrm{~V}^{v N} \\
\mathrm{~V}^{S v} & \mathrm{~V}^{S S} & \mathrm{~V}^{S N} \\
\mathrm{~V}^{N v} & \mathrm{~V}^{N S} & \mathrm{~V}^{N N}
\end{array}\right)_{\alpha i}\left(\begin{array}{c}
v_{i} \\
S_{i} \\
N_{i}
\end{array}\right) \\
& =\left(\begin{array}{ccc}
U_{\mathrm{PMNS}} & \frac{1}{m_{S}} M_{D} U_{\text {PMNS }}^{*} & \frac{v_{L}}{v_{R}} M_{D} U_{\text {PMNS }}^{-1} m_{v} \text { diag. }^{-1} \\
\frac{1}{m_{S}} M_{D}^{\dagger} U_{\mathrm{PMNS}} & U_{\mathrm{PMNS}}^{*} & \frac{v_{L}}{v_{R}} m_{S} U_{\text {PMNS }}^{-1} m_{v}^{\text {diag. }}{ }^{-1} \\
\mathbb{O} & \frac{v_{L}}{v_{R}} m_{S} U_{\mathrm{PMNS}}^{-1} m_{v}^{\text {diag. }}{ }^{-1} & U_{\mathrm{PMNS}}
\end{array}\right)_{\alpha i}\left(\begin{array}{c}
v_{i} \\
S_{i} \\
N_{i}
\end{array}\right)
\end{aligned}
$$

\section{ALTERNATIVE FORMULATION OF LEFT-RIGHT SYMMETRIC MODEL: UNIVERSAL SEESAW}

Among the various alternative formulations of left-right symmetric model that have been proposed so far, the model which includes isosinglet vector like fermions looks more upgraded. The advantages of this alternative formulation over the manifest one are the following:

- Due to the presence of vector like fermions and absence of the usual scalar bidoublet in it, the charged fermions get their masses through a seesaw mechanism called the universal seesaw instead of standard Yukawa interaction. Thus, one does not need to finetune the Yukawa couplings. The universal seesaw is named as such since both quarks and leptons get their masses through a common seesaw.

- Due to the absence of scalar bidoublet no tree level Dirac neutrino mass arises. However, the tiny Dirac neutrino mass is generated at two loop level while the righthanded interactions still lie at $\mathrm{TeV}$ scale, as shown in Figure 2.

- The same set of symmetries offer the ambiance to address the issue of weak and strong CP-violation.

- The scalar sector of the model is too simple which consists of two isodoublets.

\subsection{Left-Right Symmetry With Vector-Like Fermions and Universal Seesaw}

The fermion content of this model includes the usual quarks and leptons,

$$
\begin{aligned}
& q_{L}=\left(\begin{array}{l}
u_{L} \\
d_{L}
\end{array}\right) \sim(2,1,1 / 3,3), q_{R}=\left(\begin{array}{l}
u_{R} \\
d_{R}
\end{array}\right) \sim(1,2,1 / 3,3), \\
& \ell_{L}=\left(\begin{array}{l}
v_{L} \\
e_{L}
\end{array}\right) \sim(2,1,-1,1), \ell_{R}=\left(\begin{array}{l}
v_{R} \\
e_{R}
\end{array}\right) \sim(1,2,-1,1),
\end{aligned}
$$

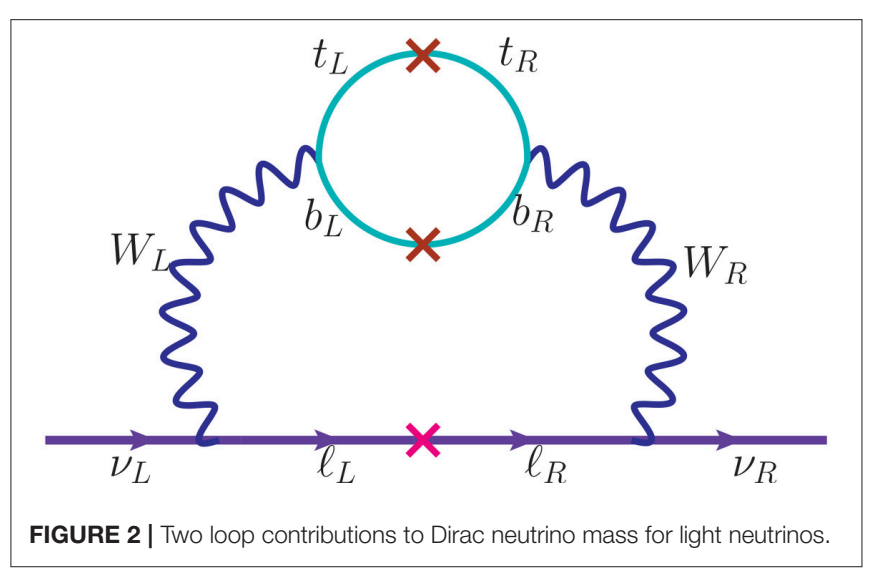


and the additional vector-like quarks and charged leptons [6270]

$$
\begin{gathered}
U_{L, R} \sim(1,1,4 / 3,3), \quad D_{L, R} \sim(1,1,-2 / 3,3), \\
E_{L, R} \sim(1,1,-2,1) .
\end{gathered}
$$

To this new setup of left-right symmetric model, we add vector like neutral lepton in the fermion sector and a singlet scalar in the Higgs sector. The purpose behind the inclusion of vector like neutral lepton is to allow seesaw mechanism for light neutrinos leading to Dirac neutrino mass. Similarly, the scalar singlet is introduced to give consistent vacuum stability in the scalar sector. The particle content and the relevant transformations under the LRSM gauge group are shown in Table 4.

We now extend the standard LRSM framework having isosinglet vector-like copies of fermions with additional neutral vector like fermions [71-75]. This kind of a vector-like fermion spectrum is very naturally embedded in gauged flavor groups with left-right symmetry [76] or quark-lepton symmetric models [77].

The relevant Yukawa part of the Lagrangian is given by

$$
\begin{aligned}
\mathcal{L}= & -\sum_{X}\left(\lambda_{S X X} S \bar{X} X+M_{X} \bar{X} X\right)-\left(\lambda_{U}^{L} \tilde{H}_{L} \bar{q}_{L} U_{R}+\lambda_{U}^{R} \tilde{H}_{R} \bar{q}_{R} U_{L}\right. \\
& +\lambda_{D}^{L} H_{L} \bar{q}_{L} D_{R}+\lambda_{D}^{R} H_{R} \bar{q}_{R} D_{L}+\lambda_{E}^{L} H_{L} \bar{\ell}_{L} E_{R}+\lambda_{E}^{R} H_{R} \bar{\ell}_{R} E_{L} \\
& \left.+\lambda_{N}^{L} \tilde{H}_{L} \bar{\ell}_{L} N_{R}+\lambda_{N}^{R} \tilde{H}_{R} \bar{\ell}_{R} N_{L}+\text { h.c. }\right),
\end{aligned}
$$

where the summation is over $X=U, D, E, N$ and we suppress flavor and color indices on the fields and couplings. $\tilde{H}_{L, R}$ denotes $\tau_{2} H_{L, R}^{*}$, where $\tau_{2}$ is the usual second Pauli matrix. We would like to stress that Parity Symmetry is present in order to distinguish between for instance $N_{R}$ and $N_{L}$, otherwise extra terms in the Lagrangian Equation (64) would appear with the vector-like fermions Left and Right exchanged.

The LRSM gauge group breaks to the SM gauge group when $H_{R}(1,2,-1)$ acquires a VEV and the SM gauge group breaks to $U(1)_{\mathrm{EM}}$ when $H_{L}(2,1,-1)$ acquires a VEV. However, parity can break either at $\mathrm{TeV}$ scale or at a much higher scale $M_{P}$. For the latter case the Yukawa couplings can be different for right-type and left-type Yukawa terms $\left(\lambda_{X}^{R} \neq \lambda_{X}^{L}\right)$ because of the renormalization group running below $M_{P}$. Consequently, we will distinguish the left and right handed couplings explicitly with the subscripts $L$ and $R$. We use the VEV normalizations $\left\langle H_{L}\right\rangle=\left(0, v_{L}\right)^{T}$ and $\left\langle H_{R}\right\rangle=\left(0, v_{R}\right)^{T}$. The scale of $v_{R}$ has to lie between at around a few $\mathrm{TeV}$ (depending on the right-handed gauge coupling) to suit the experimental searches for the heavy right-handed $W_{R}$ boson at colliders and at low energies.

Since the particle spectrum does not contain a bidoublet Higgs, Dirac mass terms for the SM fermions can not be written and the charged fermion mass matrices assume a seesaw structure. Alternatively, a Higgs bidoublet $\Phi$ can be introduced along with $H_{L, R}$.

After symmetry breaking, the mass matrices for the fermions are given by

$$
M_{u U}=\left(\begin{array}{cc}
0 & \lambda_{U}^{L} v_{L} \\
\lambda_{U}^{R} v_{R} & M_{U}
\end{array}\right), M_{d D}=\left(\begin{array}{cc}
0 & \lambda_{D}^{L} v_{L} \\
\lambda_{D}^{R} v_{R} & M_{D}
\end{array}\right)
$$

TABLE 4 | LRSM representations of extended field content.

\begin{tabular}{lcccc}
\hline Field & $\boldsymbol{S U}_{(\mathbf{2})_{\mathbf{L}}}$ & $\boldsymbol{S U}(\mathbf{2})_{\boldsymbol{R}}$ & $\boldsymbol{B}-\boldsymbol{L}$ & $\boldsymbol{S U}(\mathbf{3})_{\boldsymbol{C}}$ \\
\hline$q_{L}$ & 2 & 1 & $1 / 3$ & 3 \\
$q_{R}$ & 1 & 2 & $1 / 3$ & 3 \\
$\ell_{L}$ & 2 & 1 & -1 & 1 \\
$\ell_{R}$ & 1 & 2 & -1 & 1 \\
\hline$U_{L, R}$ & 1 & 1 & $4 / 3$ & 3 \\
$D_{L, R}$ & 1 & 1 & $-2 / 3$ & 3 \\
$E_{L, R}$ & 1 & 1 & -2 & 1 \\
$N_{L, R}$ & 1 & 1 & 0 & 1 \\
\hline$H_{L}$ & 2 & 1 & 1 & 1 \\
$H_{R}$ & 1 & 2 & 1 & 1 \\
$S$ & 1 & 1 & 0 & 1 \\
\hline
\end{tabular}

$$
M_{e E}=\left(\begin{array}{cc}
0 & \lambda_{E}^{L} v_{L} \\
\lambda_{E}^{R} v_{R} & M_{E}
\end{array}\right), M_{\nu N}=\left(\begin{array}{cc}
0 & \lambda_{N}^{L} v_{L} \\
\lambda_{N}^{R} v_{R} & M_{N}
\end{array}\right),
$$

The mass eigenstates can be found by rotating the mass matrices via left and right orthogonal transformations $O^{L, R}$ (we assume all parameters to be real). For example, the up quark diagonalization yields $O_{U}^{L T} \cdot M_{u U} \cdot O_{U}^{R}=\operatorname{diag}\left(\hat{m}_{u}, \hat{M}_{U}\right)$. Up to leading order in $\lambda_{U}^{L} v_{L}$, the resulting up-quark masses are

$$
\hat{M}_{U} \approx \sqrt{M_{U}^{2}+\left(\lambda_{U}^{R} v_{R}\right)^{2}}, \quad \hat{m}_{u} \approx \frac{\left(\lambda_{U}^{L} v_{L}\right)\left(\lambda_{U}^{R} v_{R}\right)}{\hat{M}_{U}},
$$

and the mixing angles $\theta_{U}^{L, R}$ parametrizing $O_{U}^{L, R}$,

$$
\tan \left(2 \theta_{U}^{L}\right) \approx \frac{2\left(\lambda_{U}^{L} v_{L}\right) M_{U}}{M_{U}^{2}+\left(\lambda_{U}^{R} v_{R}\right)^{2}}, \tan \left(2 \theta_{U}^{R}\right) \approx \frac{2\left(\lambda_{U}^{R} v_{R}\right) M_{U}}{M_{U}^{2}-\left(\lambda_{U}^{R} v_{R}\right)^{2}}
$$

The other fermion masses and mixings are given analogously. For an order of magnitude estimate one may approximate the phenomenologically interesting regime with the limit $\lambda_{U}^{R} v_{R} \rightarrow$ $M_{U}$ in which case the mixing angles approach $\theta_{U}^{L} \rightarrow \hat{m}_{u} / \hat{M}_{U}$ and $\theta_{U}^{R} \rightarrow \pi / 4$. This means that $\theta_{U}^{L}$ is negligible for all fermions but the top quark and its vector partner [72].

We here neglect the flavor structure of the Yukawa couplings $\lambda_{X}^{L, R}$ and $\lambda_{S X X}$ which will determine the observed quark and leptonic mixing. The hierarchy of SM fermion masses can be generated by either a hierarchy in the Yukawa couplings or in the masses of the of the vector like fermions.

As described above, the light neutrino masses are of Diractype as well, analogously given by

$$
\hat{m}_{v}=\frac{\lambda_{N}^{L} \lambda_{N}^{R} v_{L} v_{R}}{M_{N}}
$$

It is natural to assume that $M_{N} \gg v_{R}$, as the vector like $N$ is a singlet under the model gauge group. In this case, the scenario predicts naturally light Dirac neutrinos [76]. 


\subsection{Left-Right Symmetry With Vector-Like Fermions and Type-II Seesaw for Neutrino Masses}

In Table 5, we present the field content of this model and their transformations under the LRSM gauge group.

We implement a scalar sector consisting of $S U(2)_{L, R}$ doublets and triplets, however the conventional scalar bidoublet is absent. We use the Higgs doublets to implement the left-right and the electroweak symmetry breaking: $H_{R} \equiv\left(h_{R}^{0}, h_{R}^{-}\right)^{T} \equiv[1,2,-1,1]$ breaks the left-right symmetry, while $H_{L} \equiv\left(h_{L}^{0}, h_{L}^{-}\right)^{T} \equiv$ $[2,1,-1,1]$ breaks the electroweak symmetry once they acquire vacuum expectation values (VEVs),

$$
\left\langle H_{R}\right\rangle=\left(\begin{array}{c}
\frac{v_{R}}{\sqrt{2}} \\
0
\end{array}\right), \quad\left\langle H_{L}\right\rangle=\left(\begin{array}{c}
\frac{v_{L}}{\sqrt{2}} \\
0
\end{array}\right) .
$$

Note that the present framework requires only doublet Higgs fields for spontaneous symmetry breaking. However, in the absence of a Higgs bidoublet, we use the vector-like new fermions to generate correct charged fermion masses through a universal seesaw mechanism. For the neutrinos we note that in the absence of a scalar bidoublet there is no Dirac mass term for light neutrinos and without scalar triplets no Majorana masses are generated either. To remedy this fact we introduce additional scalar triplets $\Delta_{L}$ and $\Delta_{R}$,

$$
\Delta_{L, R}=\left(\begin{array}{cc}
\delta_{L, R}^{+} / \sqrt{2} & \delta_{L, R}^{++} \\
\delta_{L, R}^{0} & -\delta_{L, R}^{+} / \sqrt{2}
\end{array}\right),
$$

which transform as $\Delta_{L} \equiv[3,1,2,1]$ and $\Delta_{R} \equiv[1,3,2,1]$, respectively. They generate Majorana masses for the light and heavy neutrinos although they are not essential in spontaneous symmetry breaking here. The particle content of the model is shown in Table 5. In the presence of the Higgs triplets, the manifestly Left-Right symmetric scalar potential has the form

$$
\begin{aligned}
\mathcal{L} & =\left(D_{\mu} H_{L}\right)^{\dagger} D^{\mu} H_{L}+\left(D_{\mu} H_{R}\right)^{\dagger} D^{\mu} H_{R} \\
& +\left(D_{\mu} \Delta_{L}\right)^{\dagger} D^{\mu} \Delta_{L}+\left(D_{\mu} \Delta_{R}\right)^{\dagger} D^{\mu} \Delta_{R} \\
& -\mathcal{V}\left(H_{L}, H_{R}, \Delta_{L}, \Delta_{R}\right),
\end{aligned}
$$

TABLE 5 | Field content of the LRSM with universal seesaw.

\begin{tabular}{lcccc}
\hline Field & $\boldsymbol{S U}(\mathbf{2})_{\boldsymbol{L}}$ & $\boldsymbol{S U}(\mathbf{2})_{\boldsymbol{R}}$ & $\boldsymbol{B}-\boldsymbol{L}$ & $\boldsymbol{S U}(\mathbf{3})_{\boldsymbol{C}}$ \\
\hline$Q_{L}$ & 2 & 1 & $1 / 3$ & 3 \\
$Q_{R}$ & 1 & 2 & $1 / 3$ & 3 \\
$\ell_{L}$ & 2 & 1 & -1 & 1 \\
$\ell_{R}$ & 1 & 2 & -1 & 1 \\
\hline$U_{L, R}$ & 1 & 1 & $4 / 3$ & 3 \\
$D_{L, R}$ & 1 & 1 & $-2 / 3$ & 3 \\
$E_{L, R}$ & 1 & 1 & -2 & 1 \\
\hline$H_{L}$ & 2 & 1 & -1 & 1 \\
$H_{R}$ & 1 & 2 & -1 & 1 \\
$\Delta_{L}$ & 3 & 1 & 2 & 1 \\
$\Delta_{R}$ & 1 & 3 & 2 & 1 \\
\hline
\end{tabular}

where the scalar potential is given by

$$
\begin{aligned}
\mathcal{V}\left(H_{L}, H_{R}, \Delta_{L}, \Delta_{R}\right)=-\mu_{1}^{2}\left(H_{L}^{\dagger} H_{L}\right)-\mu_{2}^{2}\left(H_{R}^{\dagger} H_{R}\right) \\
\quad+\lambda_{1}\left(H_{L}^{\dagger} H_{L}\right)^{2}+\lambda_{2}\left(H_{R}^{\dagger} H_{R}\right)^{2}+\beta_{1}\left(H_{L}^{\dagger} H_{L}\right)\left(H_{R}^{\dagger} H_{R}\right) \\
\quad-\mu_{3}^{2} \operatorname{Tr}\left(\Delta_{L}^{\dagger} \Delta_{L}\right)-\mu_{4}^{2} \operatorname{Tr}\left(\Delta_{R}^{\dagger} \Delta_{R}\right) \\
\quad+\lambda_{3} \operatorname{Tr}\left(\Delta_{L}^{\dagger} \Delta_{L}\right)^{2}+\lambda_{4} \operatorname{Tr}\left(\Delta_{R}^{\dagger} \Delta_{R}\right)^{2} \\
\quad+\beta_{2} \operatorname{Tr}\left(\Delta_{L}^{\dagger} \Delta_{L}\right) \operatorname{Tr}\left(\Delta_{R}^{\dagger} \Delta_{R}\right) \\
\quad+\rho_{1}\left(\operatorname{Tr}\left(\Delta_{L}^{\dagger} \Delta_{L}\right)\left(H_{R}^{\dagger} H_{R}\right)+\operatorname{Tr}\left(\Delta_{R}^{\dagger} \Delta_{R}\right)\left(H_{L}^{\dagger} H_{L}\right)\right) \\
\quad+\rho_{2}\left(\operatorname{Tr}\left(\Delta_{L}^{\dagger} \Delta_{L}\right)\left(H_{L}^{\dagger} H_{L}\right)+\operatorname{Tr}\left(\Delta_{R}^{\dagger} \Delta_{R}\right)\left(H_{R}^{\dagger} H_{R}\right)\right) \\
\quad+\rho_{3}\left(H_{L}^{\dagger} \Delta_{L}^{\dagger} \Delta_{L} H_{L}+H_{R}^{\dagger} \Delta_{R}^{\dagger} \Delta_{R} H_{R}\right) \\
\quad+\mu\left(H_{L}^{T} i \sigma_{2} \Delta_{L} H_{L}+H_{R}^{T} i \sigma_{2} \Delta_{R} H_{R}\right)+\text { h.c. } \cdots .
\end{aligned}
$$

Assigning non-zero VEV to Higgs doublets $H_{R}$ and $H_{L}$ and triplets $\Delta_{R}$ and $\Delta_{L}$,

$$
\begin{aligned}
& \left\langle H_{L}^{0}\right\rangle \equiv v_{L} / \sqrt{2}, \quad\left\langle H_{R}^{0}\right\rangle \equiv v_{R} / \sqrt{2} \\
& \left\langle\Delta_{L}^{0}\right\rangle \equiv u_{L} / \sqrt{2}, \quad\left\langle\Delta_{R}^{0}\right\rangle \equiv u_{R} / \sqrt{2}
\end{aligned}
$$

the scalar potential takes the form,

$$
\begin{aligned}
\mathcal{V}\left(\left\langle H_{L}\right\rangle,\left\langle H_{R}^{0}\right\rangle,\left\langle\Delta_{L}^{0}\right\rangle,\left\langle\Delta_{R}^{0}\right\rangle\right)= \\
\quad-\frac{1}{2} \mu_{1}^{2} v_{L}^{2}-\frac{1}{2} \mu_{2}^{2} v_{R}^{2}-\frac{1}{2} \mu_{3}^{2} u_{L}^{2}-\frac{1}{2} \mu_{4}^{2} u_{R}^{2} \\
\quad+\frac{1}{4} \lambda_{1} v_{L}^{4}+\frac{1}{4} \lambda_{2} v_{R}^{4}+\frac{1}{4} \lambda_{3} u_{L}^{4}+\frac{1}{4} \lambda_{4} u_{R}^{4} \\
\quad+\frac{1}{4} \beta_{1} v_{L}^{2} v_{R}^{2}+\frac{1}{4} \beta_{2} u_{L}^{2} u_{R}^{2}-\frac{1}{2 \sqrt{2}} \mu\left(v_{L}^{2} u_{L}+v_{R}^{2} u_{R}\right) \\
\quad+\frac{1}{4} \rho_{1}\left(v_{R}^{2} u_{L}^{2}+v_{L}^{2} u_{R}^{2}\right)+\frac{1}{4} \rho_{2}\left(v_{L}^{2} u_{L}^{2}+v_{R}^{2} u_{R}^{2}\right)+\cdots
\end{aligned}
$$

As non-zero VEV $\left\langle H_{R}^{0}\right\rangle=v_{R}$ breaks LRSM to SM at high scale and $\left\langle H_{L}^{0}\right\rangle=v_{L}$ breaks SM down to low energy at electroweak scale, we consider $v_{L} \neq v_{R}$. We chose the induced VEVs for scalar triplets much smaller than VEVs of Higgs doublets, i.e., $u_{L}, u_{R} \ll v_{L}, v_{R}$.

One can approximately write down the Higgs triplets induced VEVs as follows,

$$
u_{L}=\frac{\mu v_{L}^{2}}{M_{\delta_{L}^{0}}^{2}}, \quad u_{R}=\frac{\mu v_{R}^{2}}{M_{\delta_{R}^{0}}^{2}}
$$

\subsubsection{Fermion Masses via Universal Seesaw}

As discussed earlier, in this scheme normal Dirac mass terms for the SM fermions are not allowed due to the absence of a bidoublet Higgs. However, in the presence of vector-like copies of quark and charged lepton gauge isosinglets, the charged fermion mass matrices can assume a seesaw structure. The Yukawa interaction 
Lagrangian in this model is given by

$$
\begin{aligned}
\mathcal{L}= & -Y_{U}^{L} H_{L} \bar{Q}_{L} U_{R}+Y_{U}^{R} H_{R} \bar{Q}_{R} U_{L}+Y_{D}^{L} \tilde{H}_{L} \bar{Q}_{L} D_{R} \\
& +Y_{D}^{R} \tilde{H}_{R} \bar{Q}_{R} D_{L}+Y_{E}^{L} \tilde{H}_{L} \bar{\ell}_{L} E_{R}+Y_{E}^{R} \tilde{H}_{R} \bar{\ell}_{R} E_{L} \\
& +\frac{1}{2} f\left(\bar{\ell}_{L}^{c} i \tau_{2} \Delta_{L} \ell_{L}+\bar{\ell}_{R}^{c} i \tau_{2} \Delta_{R} \ell_{R}\right) \\
& -M_{U} \bar{U}_{L} U_{R}-M_{D} \bar{D}_{L} D_{R}-M_{E} \bar{E}_{L} E_{R}+\text { h.c. }
\end{aligned}
$$

where we suppress the flavor and color indices on the fields and couplings. $\tilde{H}_{L, R}$ denotes $\tau_{2} H_{L, R}^{*}$, where $\tau_{2}$ is the usual second Pauli matrix. Note that there is an ambiguity regarding the breaking of parity, which can either be broken spontaneously with the leftright symmetry at around the $\mathrm{TeV}$ scale or at a much higher scale independent of the left-right symmetry breaking. In the latter case, the Yukawa couplings corresponding to the righttype and left-type Yukawa terms can be different because of the renormalization group running below the parity breaking scale, $Y_{X}^{R} \neq Y_{X}^{L}$. Thus, while writing the Yukawa terms above we distinguish the left- and right-handed couplings explicitly with the subscripts $L$ and $R$.

After spontaneous symmetry breaking we can write the mass matrices for the charged fermions as [73]

$$
\begin{gathered}
M_{u U}=\left(\begin{array}{cc}
0 & Y_{U}^{L} v_{L} \\
Y_{U}^{R} v_{R} & M_{U}
\end{array}\right), M_{d D}=\left(\begin{array}{cc}
0 & Y_{D}^{L} v_{L} \\
Y_{D}^{R} v_{R} & M_{D}
\end{array}\right), \\
M_{e E}=\left(\begin{array}{cc}
0 & Y_{E}^{L} v_{L} \\
Y_{E}^{R} v_{R} & M_{E}
\end{array}\right) .
\end{gathered}
$$

The corresponding generation of fermion masses is diagrammatically depicted in Figure 3. Note that we are interested in a scenario where the VEVs of the Higgs doublets are much larger than the VEVs of the Higgs triplets i.e., $u_{L} \ll v_{L}, u_{R} \ll v_{R}$. In the context of this work, we do not attempt to explain how the hierarchy between VEVs can be achieved.

Assuming all parameters to be real one can obtain the mass eigenstates by rotating the mass matrices via left and right orthogonal transformations $\mathcal{O}^{L, R}$. For example, up to leading order in $Y_{U}^{L} v_{L}$, the $\mathrm{SM}$ and heavy vector partner up-quark masses are

$$
m_{u} \approx Y_{U}^{L} Y_{U}^{R} \frac{v_{L} v_{R}}{\hat{M}_{U}}, \quad \hat{M}_{U} \approx \sqrt{M_{U}^{2}+\left(Y_{U}^{R} v_{R}\right)^{2}}
$$

and the mixing angles $\theta_{U}^{L, R}$ in $\mathcal{O}^{L, R}$ are determined as

$$
\tan \left(2 \theta_{U}^{L, R}\right) \approx 2 Y_{U}^{L, R} \frac{v_{L, R} M_{U}}{M_{U}^{2} \pm\left(Y_{U}^{R} v_{R}\right)^{2}}
$$

The other fermion masses and mixing are obtained in an analogous manner. Note that here we have neglected the flavor structure of the Yukawa couplings $Y_{X}^{L, R}$ which will determine the observed quark and charged lepton mixings. The hierarchy of SM fermion masses can be explained by assuming either a hierarchical structure of the Yukawa couplings or a hierarchical structure of the vector-like fermion masses.

\subsubsection{Neutrino Masses and Type II Seesaw Dominance}

In the model under consideration there is no tree level Dirac mass term for the neutrinos due to the absence of a Higgs bidoublet. The scalar triplets acquire induced VEVs $\left\langle\Delta_{L}\right\rangle=u_{L}$ and $\left\langle\Delta_{R}\right\rangle=$ $u_{R}$ giving the neutral lepton mass matrix in the basis $\left(v_{L}, v_{R}\right)$ given by

$$
M_{v}=\left(\begin{array}{cc}
f u_{L} & 0 \\
0 & f u_{R}
\end{array}\right)
$$

Thus, the light and heavy neutrino masses are simply $m_{v}=f u_{L} \propto$ $M_{N}=f u_{R}$. A Dirac mass term is generated at the two-loop level via the one-loop $W$ boson mixing $\theta_{W}$ (see the next section) and the exchange of a charged lepton. It is of the order $m_{D} \lesssim$ $g_{L}^{4} /\left(16 \pi^{2}\right)^{2} m_{\tau} m_{b} m_{t} / M_{W_{R}}^{2} \approx 0.1 \mathrm{eV}$ for $M_{W_{R}} \approx 5 \mathrm{TeV}$. This is intriguingly of the order of the observed neutrino masses; as long
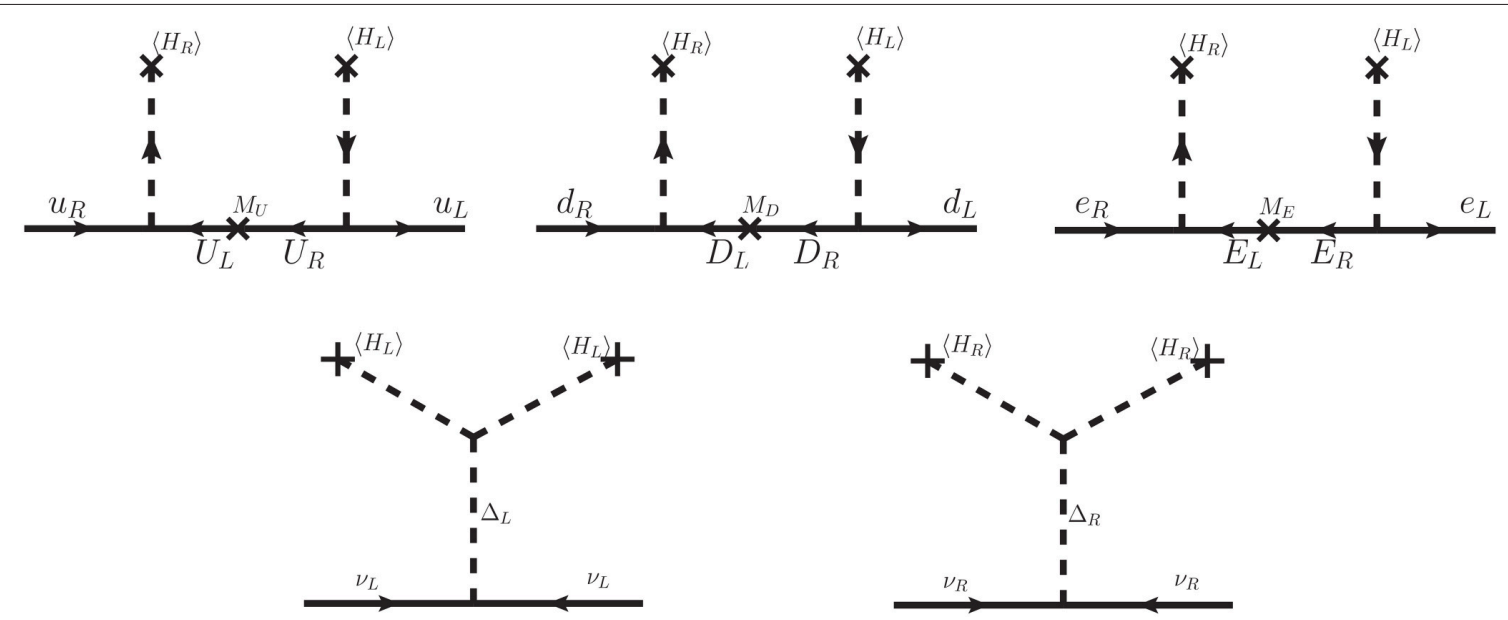

FIGURE 3 | Generation of fermion masses through universal seesaw and induced triplet VEVs. 
as the right-handed neutrinos are much heavier than the lefthanded neutrinos, the type-II seesaw dominance is preserved and the induced mixing $m_{D} / M_{N}$ is negligible. The mixing between charged gauge bosons $\theta_{W} \approx g_{L}^{2} /\left(16 \pi^{2}\right) m_{b} m_{t} / M_{W_{R}}^{2}$ is generated through the exchange of bottom and top quarks, and their vectorlike partners. This yields a very small mixing of the order $\theta_{W} \approx$ $10^{-7}$ for $\mathrm{TeV}$ scale $W_{R}$ bosons.

Incorporating three fermion generations leads to the mixing matrices for the left- and right-handed matrices which we take to be equal

$$
V_{N}=V_{v} \equiv U
$$

where $U$ is the phenomenological PMNS mixing matrix. Thus, the unmeasured mixing matrix for the right-handed neutrinos is fully determined by the left-handed counterpart. The present framework gives a natural realization of type-II seesaw providing a direct relation between light and heavy neutrinos, $M_{i} \propto m_{i}$, i.e., the heavy neutrino masses $M_{i}$ can be expressed in terms of the light neutrino masses $m_{i}$ as $M_{i}=m_{i}\left(M_{3} / m_{3}\right)$, for a normal and $M_{i}=m_{i}\left(M_{2} / m_{2}\right)$ for a inverse hierarchy of light and heavy neutrino masses.

\subsection{Implication to Neutrinoless Double Beta Decay}

As discussed earlier, there is no tree level Dirac neutrino mass term connecting light and heavy neutrinos. Consequently, the mixing between light and heavy neutrinos is vanishing at this order. Also, the mixing between the charged gauge bosons is vanishing at the tree level due to the absence of a scalar bidoublet.

The charged current interaction in the mass basis for the leptons is given by

$$
\frac{g_{L}}{\sqrt{2}} \sum_{i=1}^{3} U_{e i}\left(\overline{\ell_{L}} \gamma_{\mu} v_{i} W_{L}^{\mu}+\frac{g_{R}}{g_{L}} \bar{\ell}_{R} \gamma_{\mu} N_{i} W_{R}^{\mu}\right)+\text { h.c. }
$$

The charged current interaction for leptons leads to $0 \nu \beta \beta$ decay via the exchange of light and heavy neutrinos. There are additional contributions to $0 \nu \beta \beta$ decay due to doubly charged triplet scalar exchange. While the left-handed triplet exchange is suppressed because of its small induced VEV, the right-handed triplet can contribute sizeably to $0 \nu \beta \beta$ decay.

Before numerical estimation, let us point out the mass relations between light and heavy neutrinos under natural typeII seesaw dominance. For a hierarchical pattern of light neutrinos the mass eigenvalues are given as $m_{1}<m_{2} \ll m_{3}$. The lightest neutrino mass eigenvalue is $m_{1}$ while the other mass eigenvalues are determined using the oscillation parameters as follows, $m_{2}^{2}=$ $m_{1}^{2}+\Delta m_{\text {sol }}^{2}, m_{3}^{2}=m_{1}^{2}+\Delta m_{\mathrm{atm}}^{2}+\Delta m_{\mathrm{sol}}^{2}$. On the other hand, for the inverted hierarchical pattern of the light neutrino masses $m_{3} \ll m_{1} \approx m_{2}$ where $m_{3}$ is the lightest mass eigenvalue while other mass eigenvalues are determined by $m_{1}^{2}=m_{3}^{2}+\Delta m_{\mathrm{atm}}^{2}$, $m_{2}^{2}=m_{3}^{2}+\Delta m_{\text {sol }}^{2}+\Delta m_{\mathrm{atm}}^{2}$. The quasi-degenerate pattern of light neutrinos is $m_{1} \approx m_{2} \approx m_{3} \gg \sqrt{\Delta m_{\mathrm{atm}}^{2}}$. In any case, the heavy neutrino masses are directly proportional to the light neutrino masses.

In the present analysis, we discuss $0 \nu \beta \beta$ decay due to exchange of light neutrinos via left-handed currents, right-handed neutrinos via right handed currents as shown in Figure 4. $0 v \beta \beta$ decay can also be induced by a right handed doubly charged scalar as shown in Figure $5^{6}$. The half-life for a given isotope for these contributions is given by

$$
\left[T_{1 / 2}^{0 v}\right]^{-1}=G_{01}\left(\left|\mathcal{M}_{\nu} \eta_{\nu}\right|^{2}+\left|\left(\mathcal{M}_{N}^{\prime} \eta_{N}+\mathcal{M}_{N} \eta_{\Delta}\right)\right|^{2}\right),
$$

where $G_{01}$ corresponds to the standard $0 v \beta \beta$ phase space factor, the $\mathcal{M}_{i}$ correspond to the nuclear matrix elements for the different exchange processes and $\eta_{i}$ are dimensionless parameters determined below.

\section{Light Neutrinos}

The lepton number violating dimensionless particle physics parameter derived from $0 \nu \beta \beta$ decay due to the standard mechanism via the exchange of light neutrinos is

$$
\eta_{v}=\frac{1}{m_{e}} \sum_{i=1}^{3} U_{e i}^{2} m_{i}=\frac{m_{\mathrm{ee}}^{v}}{m_{e}} .
$$

Here, $m_{e}$ is the electron mass and the effective $0 \nu \beta \beta$ mass is explicitly given by

$$
m_{\mathrm{ee}}^{v}=\left|c_{12}^{2} c_{13}^{2} m_{1}+s_{12}^{2} c_{13}^{2} m_{2} e^{i \alpha}+s_{13}^{2} m_{3} e^{i \beta}\right|,
$$

with the sine and cosine of the oscillation angles $\theta_{12}$ and $\theta_{13}$, $c_{12}=\cos \theta_{12}$, etc. and the unconstrained Majorana phases $0 \leq$ $\alpha, \beta<2 \pi$.

\section{Right-Handed Neutrinos}

The contribution to $0 \nu \beta \beta$ decay arising from the purely righthanded currents via the exchange of right-handed neutrinos generally results in the lepton number violating dimensionless particle physics parameter

$$
\eta_{N}=m_{p}\left(\frac{g_{R}}{g_{L}}\right)^{4}\left(\frac{M_{W_{L}}}{M_{W_{R}}}\right)^{4} \sum_{i=1}^{3} \frac{U_{e i}^{2} M_{i}}{|p|^{2}+M_{i}^{2}} .
$$

The virtual neutrino momentum $|p|$ is of the order of the nuclear Fermi scale, $p \approx 100 \mathrm{MeV} . m_{p}$ is the proton mass and for the manifest LRSM case we have $g_{L}=g_{R}$, or else the new contributions are rescaled by the ratio between these two couplings. We in general consider right-handed neutrinos that can be either heavy or light compared to nuclear Fermi scale.

${ }^{6} \mathrm{~A}$ detailed discussion of $0 \nu \beta \beta$ decay within LRSMs can be found e.g., in Mohapatra and Senjanovic [19], Mohapatra and Vergados [78], Hirsch et al. [79], Tello et al. [80], Chakrabortty et al. [81], Patra [75], Awasthi et al. [60], Barry and Rodejohann [82], Bhupal Dev et al. [83], Ge et al. [84], Awasthi et al. [85], Huang and Lopez-Pavon [86], Bhupal Dev et al. [87], Borah and Dasgupta [88], Bambhaniya et al. [89], Gu [90], Borah and Dasgupta [91] and Awasthi et al. [92] and for an early study of the effects of light and heavy Majorana neutrinos in neutrinoless double beta decay see in Halprin et al. [93]. 

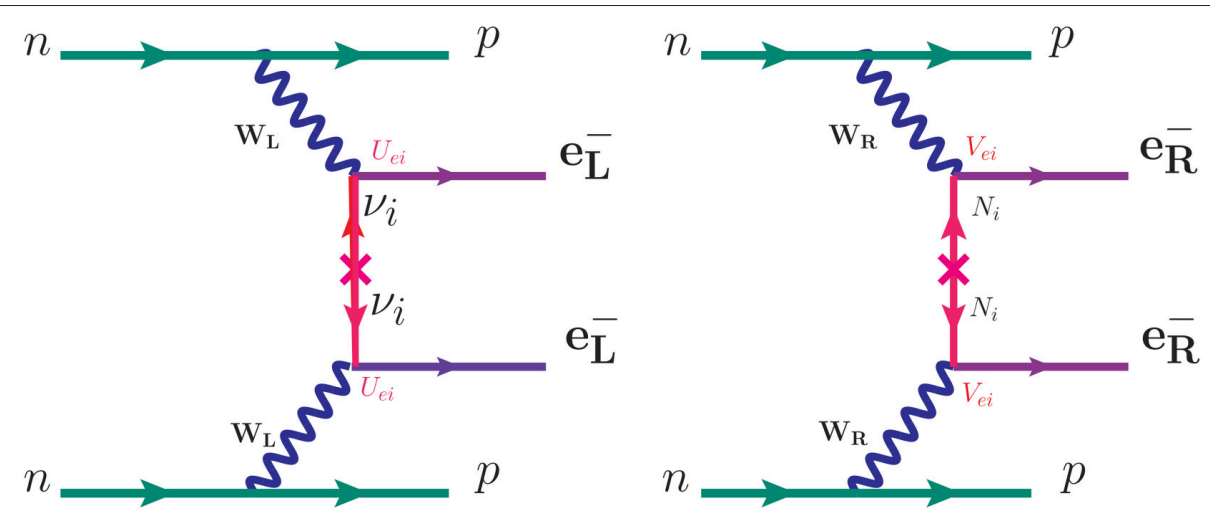

FIGURE 4 | Feynman diagrams for $0 v \beta \beta$ decay due to light left-handed and right-handed neutrinos.
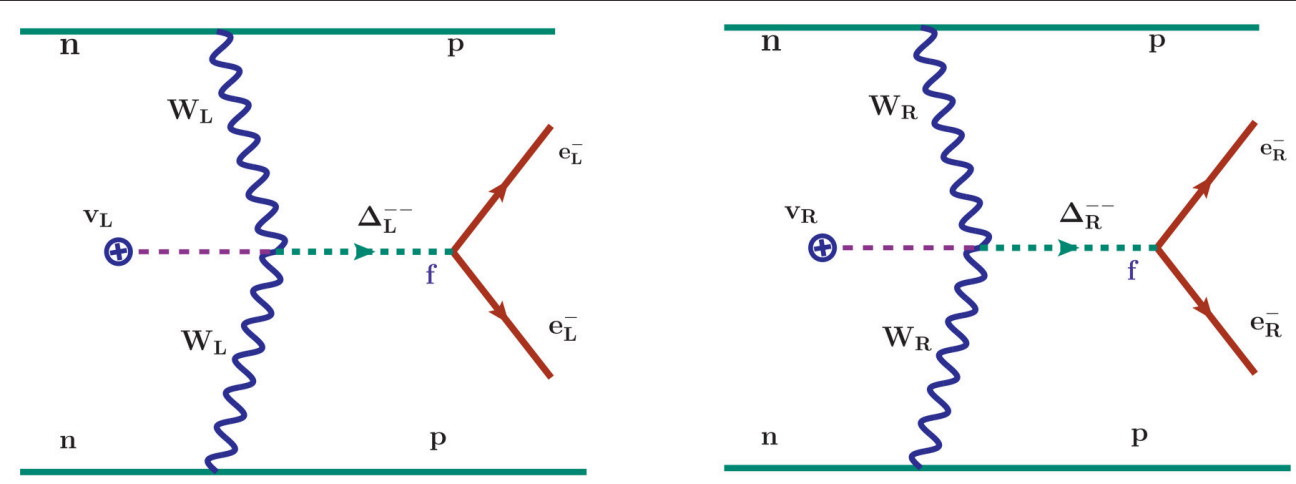

FIGURE 5 | Feynman diagrams for $0 v \beta \beta$ decay due to doubly charged scalar triplets.

If the mass of the exchanged neutrino is much higher than its momentum, $M_{i} \gg|p|$, the propagator simplifies as

$$
\frac{M_{i}}{p^{2}-M_{i}^{2}} \approx-\frac{1}{M_{i}}
$$

and the effective parameter for right-handed neutrino exchange yields

$$
\eta_{N}=m_{p}\left(\frac{g_{R}}{g_{L}}\right)^{4}\left(\frac{M_{W}}{M_{W_{R}}}\right)^{4} \sum_{i=1}^{3} \frac{U_{e i}^{2}}{M_{i}} \propto \eta_{\nu}\left(m_{i}^{-1}\right)
$$

where in the expression for $\eta_{v}\left(m_{i}^{-1}\right)$ the individual neutrino masses are replaced by their inverse values. Such a contribution clearly becomes suppressed the smaller the right-handed neutrino masses are.

On the other hand, if the mass of the neutrino is much less than its typical momentum, $M_{i} \ll|p|$, the propagator simplifies in the same way as for the light neutrino exchange,

$$
P_{R} \frac{\not p+M_{i}}{p^{2}-M_{i}^{2}} P_{R} \approx \frac{M_{i}}{p^{2}}
$$

because both currents are right-handed. As a result, the $0 \nu \beta \beta$ decay contribution leads to the dimensionless parameter

$$
\eta_{N}=\frac{m_{p}}{|p|^{2}}\left(\frac{g_{R}}{g_{L}}\right)^{4}\left(\frac{M_{W}}{M_{W_{R}}}\right)^{4} \sum_{i=1}^{3} U_{e i}^{2} M_{i} \propto \eta_{\nu} .
$$

This is proportional to the standard parameter $\eta_{v}$ but in the case of very light right-handed neutrinos, e.g., $M_{i} \approx m_{i}$, the contribution becomes negligible because of the strong suppression with the heavy right-handed $W$ boson mass.

In general, we consider right-handed neutrinos both lighter and heavier than $100 \mathrm{MeV}$ and use (86) to calculate the contribution. In addition, the relevant nuclear matrix element changes; for $M_{i} \gg 100 \mathrm{MeV}$ it approaches $\mathcal{M}_{N}^{\prime} \rightarrow \mathcal{M}_{N}$ whereas for $M_{i} \ll 100 \mathrm{MeV}$ it approaches $\mathcal{M}_{N}^{\prime} \rightarrow \mathcal{M}_{\nu}$. For intermediate values, we use a simple smooth interpolation scheme within the regime $10 \mathrm{MeV}-1 \mathrm{GeV}$, which yields a sufficient accuracy for our purposes.

\section{Right-Handed Triplet Scalar}

Finally, the exchange of a doubly charged right-handed triplet scalar shown in Figure 5 (where doubly charged left-handed triplet scalar contributes negligible and thus, neglected from the 
TABLE 6 | Phase space factor $G_{01}$ and ranges of nuclear matrix elements for light and heavy neutrino exchange for the isotopes ${ }^{76} \mathrm{Ge}$ and ${ }^{136} \mathrm{Xe}$ [95].

\begin{tabular}{cccc}
\hline Isotope & $\boldsymbol{G}_{\mathbf{0 1}}\left(\mathrm{yr}^{-\mathbf{1}}\right)$ & $\mathcal{M}_{\boldsymbol{v}}$ & $\mathcal{M}_{\boldsymbol{N}}$ \\
\hline${ }^{76} \mathrm{Ge}$ & $5.77 \times 10^{-15}$ & $2.58-6.64$ & $233-412$ \\
${ }^{136} \mathrm{Xe}$ & $3.56 \times 10^{-14}$ & $1.57-3.85$ & $164-172$ \\
\hline
\end{tabular}

present discussion) gives

$$
\eta_{\Delta}=\frac{m_{p}}{M_{\delta_{R}^{--}}^{2}}\left(\frac{g_{R}}{g_{L}}\right)^{4}\left(\frac{M_{W}}{M_{W_{R}}}\right)^{4} \sum_{i=1}^{3} U_{e i}^{2} M_{i} \propto \eta_{\nu} .
$$

This expression is also proportional to the standard $\eta_{v}$ because the relevant coupling of the triplet scalar is proportional to the right-handed neutrino mass.

\section{Numerical Estimate}

In the following, we numerically estimate the half-life for $0 v \beta \beta$ decay of the isotope ${ }^{136} \mathrm{Xe}$ as shown in Figure 6. We use the current values of masses and mixing parameters from neutrino oscillation data reported in the global fits taken from GonzalezGarcia et al. [94]. For the $0 \nu \beta \beta$ phase space factors and nuclear matrix elements we use the values given in Table 6. In Figure 6, we show the dependence of the $0 v \beta \beta$ decay half-life on the lightest neutrino mass, i.e., $m_{1}$ for normal and $m_{3}$ for inverse hierarchical neutrinos. The other model parameters are fixed as

$$
g_{R}=g_{L}, M_{W_{R}}=M_{\delta_{R}^{--}} \approx 5 \mathrm{TeV}, M_{N}^{\text {heaviest }}=1 \mathrm{TeV}
$$

The lower limit on lightest neutrino mass is derived to be $m_{<} \approx 0.9 \mathrm{meV}, 0.01 \mathrm{meV}$ for $\mathrm{NH}$ and $\mathrm{IH}$ pattern of light neutrino masses respectively by saturating the KamLAND-Zen experimental bound.

As for the experimental constraints, we use the current best limits at $90 \%$ C.L., $T_{1 / 2}^{0 v}\left({ }^{136} \mathrm{Xe}\right)>1.07 \times 10^{26} \mathrm{yr}$ and $T_{1 / 2}^{0 v}\left({ }^{76} \mathrm{Ge}\right)>$ $2.1 \times 10^{25} \mathrm{yr}$ from KamLAND-Zen [96] and the GERDA Phase I [97], respectively. Representative for the sensitivity of future $0 \nu \beta \beta$ experiments, we use the expected reach of the planned nEXO experiment, $T_{1 / 2}^{0 v}\left({ }^{136} \mathrm{Xe}\right) \approx 6.6 \times 10^{27} \mathrm{yr}$ [98]. As for the other experimental probes on the neutrino mass scale, we use the future sensitivity of the KATRIN experiment on the effective single $\beta$ decay mass $m_{\beta} \approx 0.2 \mathrm{eV}$ [99] and the current limit on the sum of neutrino masses from cosmological observations, $\Sigma_{i} m_{i} \lesssim 0.7 \mathrm{eV}[100]$.

For a better understanding of the interplay between the leftand right-handed neutrino mass scales, we show in Figure 7 the $0 \nu \beta \beta$ decay half-life as a function of the lightest neutrino mass and the heaviest neutrino mass for a normal (left) and inverse (right) neutrino mass hierarchy. The other model parameters are fixed, with right-handed gauge boson and doubly-charged scalar masses of $5 \mathrm{TeV}$. The oscillation parameters are at their best fit values and the Majorana phases are always chosen to yield the smallest rate at a given point, i.e., the longest half life. The nuclear matrix employed are at the lower end in Table 6. This altogether yields the longest, i.e., most pessimistic, prediction for the $0 v \beta \beta$

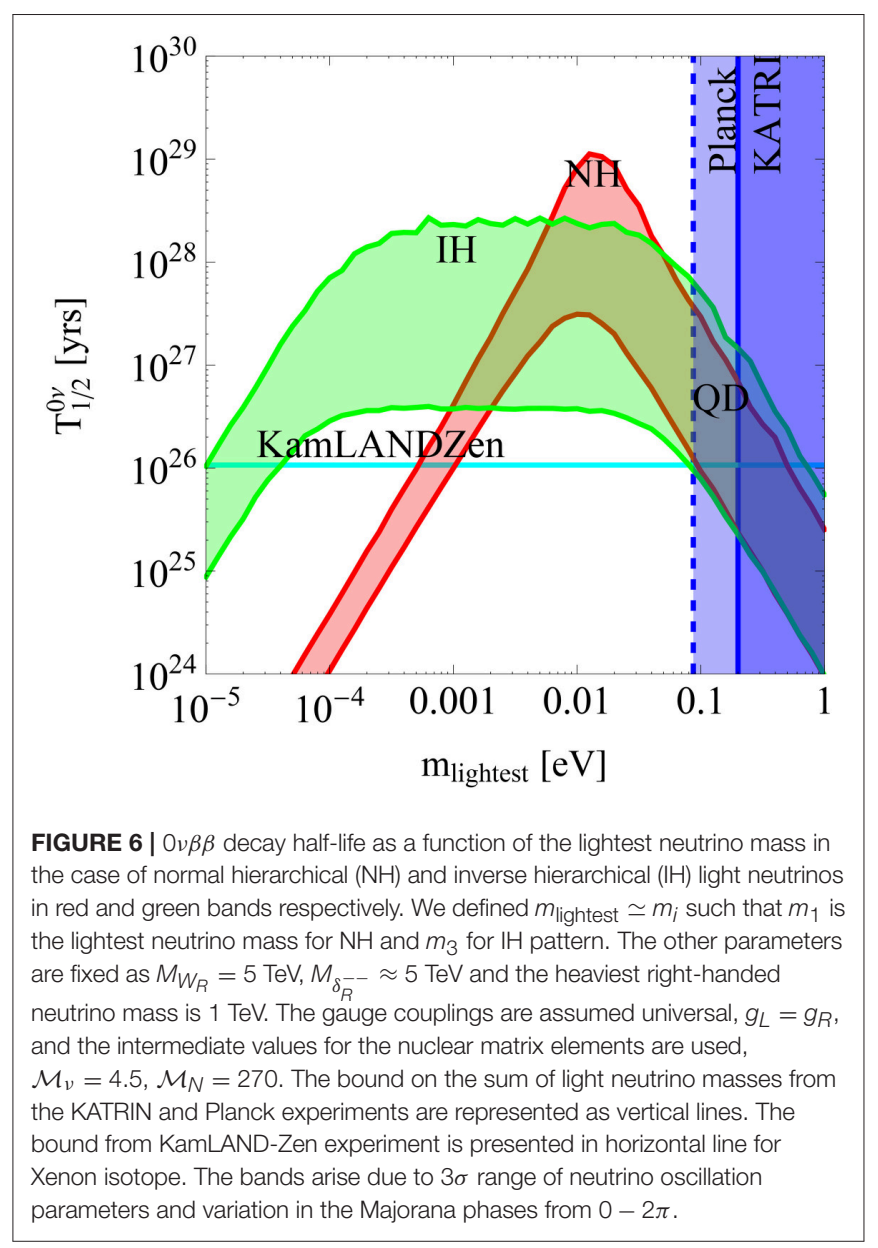

decay half-life. The red-shaded area is already excluded with a predicted half life of $10^{26} \mathrm{yr}$ or faster. As expected, this sets an upper limit on the lightest neutrino mass $m_{\text {lightest }} \lesssim 1 \mathrm{eV}$, but it also puts stringent constraints on the mass scale of the righthanded neutrinos. For an inverse hierarchy, the range $50 \mathrm{MeV} \lesssim$ $M_{2} \lesssim 5 \mathrm{GeV}$ is excluded whereas in the normal hierarchy case, large $M_{3}$ can be excluded if there is a strong hierarchy, $m_{1} \rightarrow 0$. This is due the large contribution of the lightest heavy neutrino $N_{1}$ in such a case.

\section{LEPTOGENESIS}

Cosmological observations (studies of the cosmic microwave background radiation, large scale structure data, the primordial abundances of light elements) indicate that our visible universe is dominated by matter and there is very little antimatter. The baryon asymmetry normalized to number density of photons $\left(n_{\gamma}\right)$ can be extracted out of these observations, which gives

$$
\eta(t=\text { present })=\frac{n_{B}-n_{\bar{B}}}{n_{\gamma}} \sim 10^{-10} .
$$

The astrophysical observations suggest that at an early epoch before the big-bang nucleosynthesis this asymmetry was 

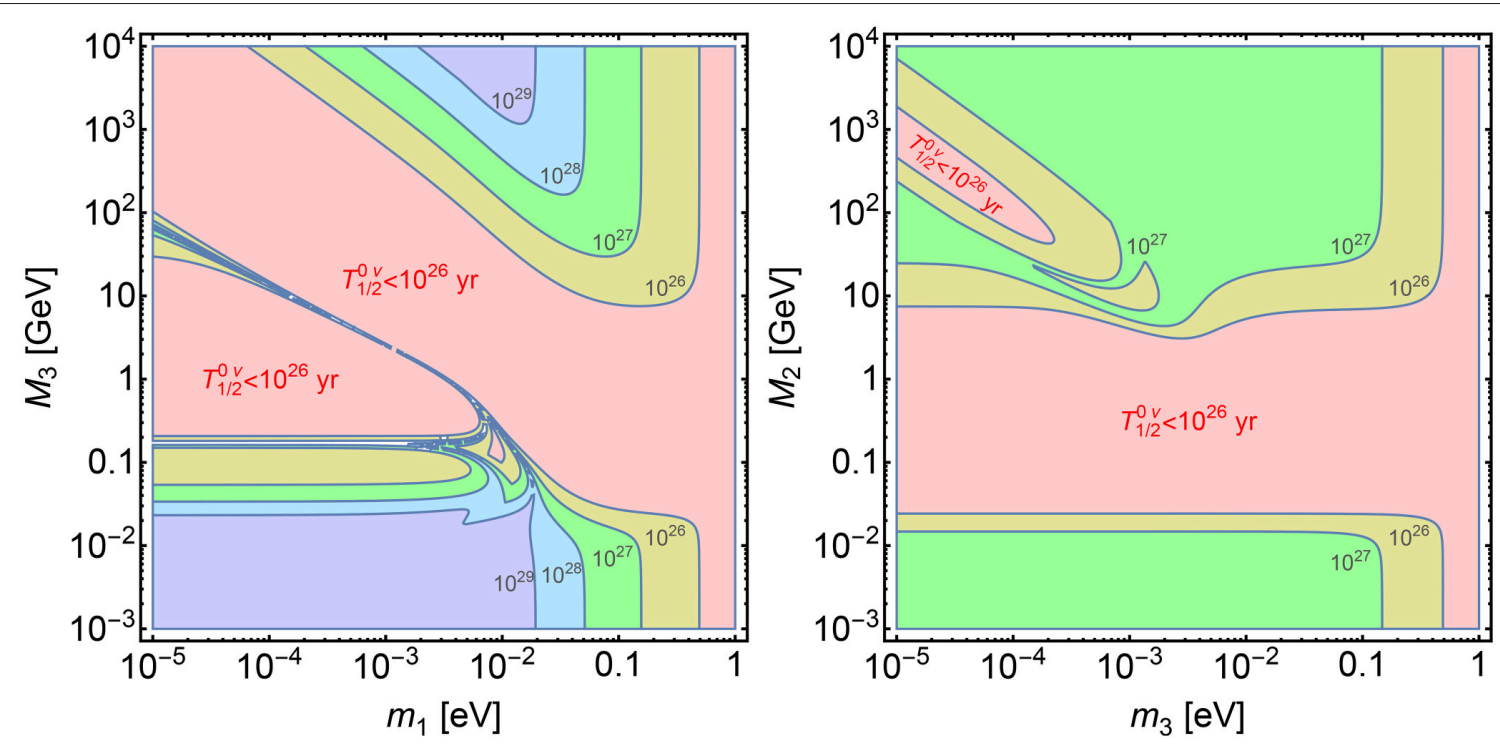

FIGURE 7 | Half-life of $0 v \beta \beta$ decay in Xe as a function of the lightest and the heaviest neutrino mass for a normal (left) and inverse (right) neutrino mass hierarchy. The contours denote the half-life in years. Best-fit oscillation data are used and the Majorana phases are chosen to yield the longest half-life. Likewise, the smallest values of the nuclear matrix elements in Table 6 are employed. The other model parameters are chosen as $g_{R}=g_{L}$ and $M_{W_{R}}=M_{\Delta}=5$ TeV.

generated. Thus, it is natural to seek an explanation for this asymmetry from the fundamental particle interactions within or beyond the SM of particle physics. There are three conditions, often called Sakharov's conditions [101], that must be met in order to generate a baryon asymmetry dynamically:

1. baryon number violation,

2. $C$ and $C P$ violation, and

3. departure from thermal equilibrium.

In principle, the SM has all the ingredients to satisfy all three conditions.

1. In the SM baryon number $B$ and lepton number $L$ are violated due to the triangle anomaly, leading to 12 -fermion processes involving nine left handed quarks (three of each generation) and three left handed leptons (one from each generation) obeying the selection rule $\Delta(B-L)=0$. These processes have a highly suppressed amplitude proportional to $e^{-4 \pi / \alpha}$ ( where $\alpha=\alpha_{\mathrm{EM}} / \sin ^{2} \theta_{W}$, with $\alpha_{\mathrm{EM}}$ being the fine structure constant and $\theta_{W}$ being the weak mixing angle) at zero temperature. However, at high temperature this suppression is lifted and these processes can be very fast.

2. The weak interactions in the $S M$ violate $C$ in a maximal way. $C P$ is also violated via the CKM phase $\delta_{C K M}$.

3. The electroweak phase transition can result in the departure from thermal equilibrium if it is sufficiently strongly first order.

However, in practice it turns out that only the first Sakharov condition is fulfilled in a satisfactory manner in the SM. The $C P$ violation coming from the CKM phase is suppressed by a factor $T_{\mathrm{EW}}^{12}$ in the denominator, where $T_{\mathrm{EW}} \sim 100 \mathrm{GeV}$ is the temperature during the electroweak phase transition.
Consequently, the $C P$ violation in the $\mathrm{SM}$ is too small to explain the observed baryon asymmetry of the universe. Furthermore, the electroweak phase transition is not first order; but just a smooth crossover.

Thus, to explain the baryon asymmetry of the universe one must go beyond the SM, either by introducing new sources of $C P$ violation and a new kind of out-of-equilibrium situations (such as the out-of-equilibrium decay of some new heavy particles) or modifying the electroweak phase transition itself. One such alternative is leptogenesis. Leptogenesis is a mechanism where a lepton asymmetry is generated before the electroweak phase transition, which then gets converted to baryon asymmetry of the universe in the presence of sphaleron induced anomalous $B+L$ violating processes, which converts any primordial $L$ asymmetry, and hence $B-L$ asymmetry, into a baryon asymmetry. A realization of leptogenesis via the decay of out-of-equilibrium heavy neutrinos transforming as singlets under the SM gauge group was proposed in Fukugita and Yanagida [21]. The Yukawa couplings provide the $C P$ through interference between tree level and one-loop decay diagrams. The departure from thermal equilibrium occurs when the Yukawa interactions are sufficiently slow ${ }^{7}$. The lepton number violation in this scenario comes from

\footnotetext{
${ }^{7}$ The out of equilibrium condition can be understood as follows. In thermal equilibrium the expectation value of the baryon number can be written as $\langle B\rangle=$ $\operatorname{Tr}\left[\mathrm{Be}^{-\beta \mathrm{H}}\right] / \operatorname{Tr}\left[\mathrm{e}^{-\beta \mathrm{H}}\right]$, where $\beta$ is the inverse temperature. Since particles and anti particles have opposite baryon number, $B$ is odd under $C$ operation, while it is even under $P$ and $T$ operations. Thus, $C P T$ conservation implies a vanishing total baryon number since $B$ is odd and $H$ is even under $C P T$, unless there is a non-vanishing chemical potential. Assuming a non-vanishing chemical potential implies that the above equation for the expectation value of the baryon number is no longer valid and the baryon number density departs from the equilibrium distribution. This is achieved when the interaction rate is very slow compared to the expansion rate of the universe.
} 
the Majorana masses of the heavy neutrinos. The generated lepton asymmetry then gets partially converted to baryon asymmetry in the presence of sphaleron induced anomalous $B+L$ violating interactions before the electroweak phase transition. In what follows, we will discuss the sphaleron processes and few of the most popular scenarios of leptogenesis in some detail to set the stage before discussing leptogenesis in LRSM scenarios in particular.

\subsection{Anomalous $B+L$ Violating Processes and Relating Baryon and Lepton Asymmetries}

In the SM both $B$ and $L$ are accidental symmetries and at the tree level these symmetries are not violated. However, the chiral nature of weak interactions gives rise to equal global anomalies for $B$ and $L$, giving a vanishing $B-L$ anomaly, but a nonvanishing axial current corresponding to $B+L$, given by t Hooft $[102,103]$

$$
\partial_{\mu} j_{(B+L)}^{\mu}=\frac{2 N_{f}}{8 \pi}\left(\alpha_{2} W_{\mu \nu}^{a} \tilde{W}^{a \mu \nu}-\alpha_{1} B_{\mu \nu} \tilde{B}^{\mu \nu}\right),
$$

where $W_{\mu \nu}^{a}$ and $B_{\mu \nu}$ are the $S U(2)_{L}$ and $U(1)_{Y}$ field strength tensors and $N_{f}$ is the number of fermion generations. The corresponding $B+L$ violation can obtained by integrating the divergence of the $B+L$ current, which is related to the change in the topological charges of the gauge field

$$
\Delta(B+L)=\int d^{4} x \partial^{\mu} j_{\mu}^{(B+L)}=2 N_{f} \Delta N_{\mathrm{cs}},
$$

where $N_{\mathrm{cs}}= \pm 1, \pm 2, \cdots$ corresponds to the topological charge of gauge fields, called the Chern-Simons number. In the SM there are three generations of fermions $\left(N_{f}=3\right)$, leading to $\Delta B=$ $\Delta L=3 N_{\mathrm{cs}}$, thus the vacuum to vacuum transition changes $B$ and $L$ by multiples of 3 units. At the lowest order, one has the $B+L$ violating effective operator

$$
\mathcal{O}(B+L)=\prod_{i=123}\left(q_{L i} q_{L i} q_{L i} l_{L i}\right),
$$

which gives rise to 12 -fermion sphaleron induced transitions, such as

$$
|\mathrm{vac}\rangle \rightarrow\left[u_{L} u_{L} d_{L} e_{L}^{-}+c_{L} c_{L} s_{L} \mu_{L}^{-}+t_{L} t_{L} b_{L} \tau_{L}^{-}\right]
$$

At zero temperature the transition rate is suppressed by $e^{-4 \pi / \alpha}=$ $\mathcal{O}\left(10^{-165}\right)[102,103]$. However, when the temperature is larger than the barrier height, this Boltzmann suppression disappears and $B+L$ violating transitions can occur at a significant rate [104]. In the symmetric phase, when the temperature is grater than the electroweak phase transition temperature, $T \geq T_{\mathrm{EW}}$, the transition rate per unit volume is [105-108]

$$
\frac{\Gamma_{B-L}}{V} \sim \alpha^{5} \ln \alpha^{-1} T^{4}
$$

where $\alpha=\alpha_{\mathrm{EM}} / \sin ^{2} \theta_{W}$, with $\alpha_{\mathrm{EM}}$ being the fine structure constant and $\theta_{W}$ being the weak mixing angle.
An account of the $B-L$ symmetry getting converted to a baryon asymmetry via an analysis of the chemical potential can be found in Khlebnikov and Shaposhnikov [109], Harvey and Turner [110] and Sarkar [111]. The baryon asymmetry in terms of the $B-L$ number density can be written as

$$
\begin{aligned}
& B\left(T>T_{\mathrm{EW}}\right)=\frac{24+4 m}{66+13 m}(B-L), \\
& B\left(T<T_{\mathrm{EW}}\right)=\frac{32+4 m}{98+13 m}(B-L) .
\end{aligned}
$$

Thus, the primordial $B-L$ asymmetry gets partially converted into a baron asymmetry of the universe after the electroweak phase transition.

\subsection{Leptogenesis With Right Handed Neutrinos}

In section 2, we have discussed how adding singlet right handed neutrinos $N_{R i}$ to the SM can generate tiny seesaw masses [1420] for light neutrinos. Beyond the generation of light neutrino masses, the interaction terms

$$
\mathcal{L}_{\text {int }}=h_{\alpha i} \bar{l}_{L \alpha} \phi N_{R i}+M_{i} \overline{\left(N_{R i}\right)^{c}} N_{R i}
$$

can also provide all the ingredients necessary for realizing leptogenesis. We will work on a basis where the right handed neutrino mass matrix is real and diagonal. Furthermore we assume a hierarchical mass spectrum for the right handed neutrinos $M_{3}>M_{2}>M_{1}$. The Majorana mass term gives rise to lepton number violating decays of the right handed neutrinos

$$
\begin{aligned}
N_{R i} & \rightarrow l_{i L}+\bar{\phi}, \\
& \rightarrow l_{i L}{ }^{c}+\phi,
\end{aligned}
$$

which can generate a lepton asymmetry if there is $C P$ violation and the decay is out of equilibrium [21]. This lepton asymmetry (equivalently $B-L$ asymmetry) then gets converted to baryon asymmetry in presence of anomalous $B+L$ violating processes before the electroweak phase transition.

In the original proposal [21] and few subsequent works [112$116]$, only the $C P$ violation coming from interference of tree level and one-loop vertex diagrams, shown in Figure 8. was considered. This is somewhat analogous to the $C P$ violation in $K$ physics coming from the penguin diagram. The $C P$ asymmetry parameter corresponding to the vertex type $C P$ violation is given by

$$
\begin{aligned}
\varepsilon_{v} & \equiv \frac{\Gamma\left(N \rightarrow l \phi^{\dagger}\right)-\Gamma\left(N \rightarrow l^{c} \phi\right)}{\Gamma\left(N \rightarrow l \phi^{\dagger}\right)+\Gamma\left(N \rightarrow l^{c} \phi\right)} \\
& =-\frac{1}{8 \pi} \sum_{i=2,3} \frac{\operatorname{Im}\left[\Sigma_{\alpha}\left(h_{\alpha 1}^{*} h_{\alpha i}\right) \Sigma_{\beta}\left(h_{\beta 1}^{*} h_{\beta i}\right)\right]}{\Sigma_{\alpha}\left|h_{\alpha 1}\right|^{2}} f_{v}\left(\frac{M_{i}^{2}}{M_{1}^{2}}\right)
\end{aligned}
$$

where the loop function $f_{v}$ is defined by

$$
f_{v}(x)=\sqrt{x}\left[1-(1+x) \ln \left(\frac{1+x}{x}\right)\right] .
$$




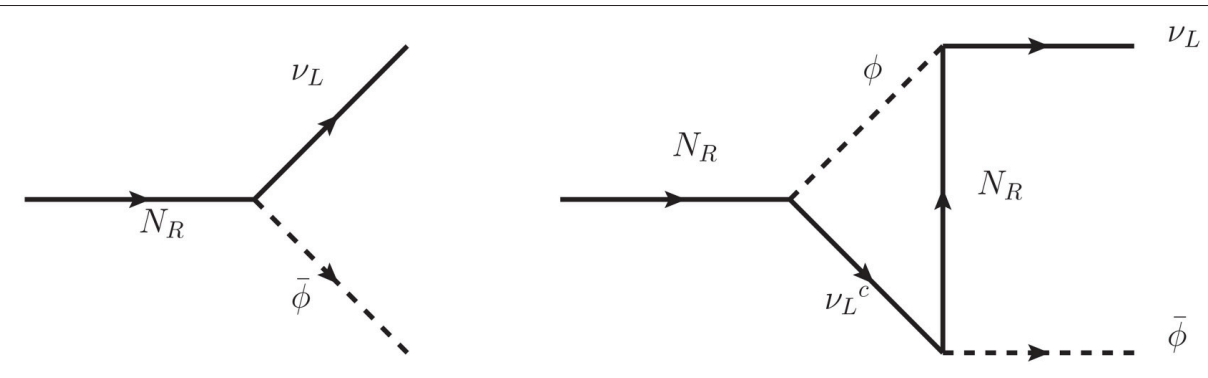

FIGURE 8 | Tree level and one-loop vertex diagrams contributing to the vertex type $C P$ violation in models with right handed neutrinos.

In the limit $M_{1} \ll M_{2}, M_{3}$ the asymmetry simplifies to

$$
\varepsilon_{v} \simeq-\frac{3}{16 \pi} \sum_{i=2,3} \frac{M_{1}}{M_{i}} \frac{\operatorname{Im}\left[\Sigma_{\alpha}\left(h_{\alpha 1}^{*} h_{\alpha i}\right) \Sigma_{\beta}\left(h_{\beta 1}^{*} h_{\beta i}\right)\right]}{\Sigma_{\alpha}\left|h_{\alpha 1}\right|^{2}} .
$$

It was later pointed out in Flanz et al. [117] and Flanz et al. [118] and confirmed rigorously in Pilaftsis [119], Pilaftsis and Resonant [120], Roulet et al. [121], Buchmuller and Plumacher [122], Flanz and Paschos [123], Hambye et al. [124] and Pilaftsis and Underwood [125], that there is another source of $C P$ violation coming from interference of tree level diagram with one-loop self-energy diagram shown in Figure 9. This $C P$ violation is similar to the $C P$ violation due to the box diagram, entering the mass matrix in $K-\bar{K}$ mixing in $K$-physics. If the heavy neutrinos decay in equilibrium, the $C P$ asymmetry coming from the selfenergy diagram due to one of the heavy neutrinos may cancel with the asymmetry from the decay of another heavy neutrino to preserve unitarity. However, in out-of-equilibrium decay of heavy neutrinos the number densities of the two heavy neutrinos differ during their decay and consequently, this cancellation is no longer present. This can be understood as the right handed neutrinos oscillating into antineutrinos of different generations, which under the condition $\Gamma$ [particle $\rightarrow$ antiparticle] $\neq$ $\Gamma$ [antiparticle $\rightarrow$ particle], can create an asymmetry in right handed neutrinos before they decay. An elementary discussion regarding how the $C P$ violation enters in Majorana mass matrix, which then generates a lepton asymmetry can be found in Sarkar [111] and Langacker et al. [126]. The basic idea is to treat the particles and the antiparticles independently. The $C P$ eigenstates $\left|N_{i}\right\rangle$ and $\left|N_{i}^{c}\right\rangle$ are no longer physical eigenstates, which evolves with time. Consequently, the physical states, which are admixtures of $\left|N_{i}\right\rangle$ and $\left|N_{i}^{c}\right\rangle$, can decay into both leptons and antileptons, giving rise to a $C P$ violation. The $C P$ asymmetry parameter coming from the interference of tree level and oneloop self-energy diagram is given by

$$
\begin{aligned}
\varepsilon_{s} & \equiv \frac{\Gamma\left(N \rightarrow l \phi^{\dagger}-N \rightarrow l^{c} \phi\right)}{\Gamma\left(N \rightarrow l \phi^{\dagger}+N \rightarrow l^{c} \phi\right)} \\
& =\frac{1}{8 \pi} \sum_{i=2,3} \frac{\operatorname{Im}\left[\Sigma_{\alpha}\left(h_{\alpha 1}^{*} h_{\alpha i}\right) \Sigma_{\beta}\left(h_{\beta 1}^{*} h_{\beta i}\right)\right]}{\Sigma_{\alpha}\left|h_{\alpha 1}\right|^{2}} f_{s}\left(\frac{M_{i}^{2}}{M_{1}^{2}}\right)
\end{aligned}
$$

where the loop function $f_{s}$ is defined by

$$
f_{s}(x)=\frac{\sqrt{x}}{1-x}
$$

When the mass difference between the right handed neutrinos is very large compared to the width, $M_{1}-M_{2} \gg \frac{1}{2} \Gamma_{N_{1,2}}$, the $C P$ asymmetries coming from vertex and self-energy diagrams are comparable. However, when two right handed neutrinos are nearly degenerate, such that their mass difference is comparable to their width, then $C P$ violation contribution coming from the self-energy diagram becomes very large (orders of magnitude larger than the $C P$ asymmetry generated by the vertex type diagram). This is often referred to as the resonance effect.

To ensure that the lightest right handed neutrino decays outof-equilibrium so that an asymmetry is generated, the out-ofequilibrium condition given by

$$
\frac{h_{\alpha 1}}{16 \pi} M_{1}<1.66 \sqrt{g_{*}} \frac{T^{2}}{m_{\mathrm{Pl}}} \quad \text { at } T=M_{1} .
$$

must be satisfied, where $g_{*}$ correspond to the effective number of relativistic degrees of freedom. This gives a lower bound $m_{N_{1}}>$ $10^{8} \mathrm{GeV}$ [127]. Though this gives us a rough estimate, in an actual calculation of the asymmetry one solves the Boltzmann equation, which takes into account both lepton number violating as well as lepton number conserving processes mediated by heavy neutrinos. The Boltzmann equation governing lepton number asymmetry $n_{L} \equiv n_{l}-n_{l}$, is given by

$$
\begin{aligned}
\frac{d n_{L}}{d t}+3 H n_{L}= & \left(\varepsilon_{v}+\varepsilon_{s}\right) \Gamma_{\psi_{1}}\left(n_{\psi_{1}}-n_{\psi_{1}}^{e q}\right) \\
& -\frac{n_{L}}{n_{\gamma}} n_{\psi_{1}}^{e q} \Gamma_{\psi_{1}}-2 n_{\gamma} n_{L}\langle\sigma|v|\rangle,
\end{aligned}
$$

where $\Gamma_{\psi_{1}}$ is the decay rate of the physical state $\left|\psi_{1}\right\rangle, n_{\psi_{1}}^{e q}$ is the equilibrium number density of $\psi_{1}$ given by

$$
n_{\psi_{1}}^{e q}=\left\{\begin{array}{cc}
s g_{*}{ }^{-1} & T \gg m_{\psi_{1}} \\
\frac{s}{g^{*}}\left(\frac{m_{\psi_{1}}}{T}\right)^{3 / 2} \exp \left(-\frac{m_{\psi_{1}}}{T}\right) & T \ll m_{\psi_{1}},
\end{array}\right.
$$

where $s$ is the entropy density. The first term on the right hand side of Equation (108) corresponds to the $C P$ violating 
contribution to the asymmetry and is the only term that generates asymmetry when $\psi_{1}$ decays out-of-equilibrium, while the second term corresponds to inverse decay of $\psi_{1}$, and the last term corresponds to $2 \leftrightarrow 2$ lepton number violating scattering process such as $l+\phi^{\dagger} \leftrightarrow l^{c}+\phi$, with $\langle\sigma|v|\rangle$ being the thermally averaged cross section. The number density of $\psi_{1}$ is governed by the Boltzmann equation

$$
\frac{d n_{\psi_{1}}}{d t}+3 H n_{\psi_{1}}=-\Gamma_{\psi_{1}}\left(n_{\psi_{1}}-n_{\psi_{1}}^{e q}\right)
$$

One often defines a parameter $K=\Gamma_{\psi_{1}}\left(T=m_{\psi_{1}}\right) / H(T=$ $\left.m_{\psi_{1}}\right)$, where the Hubble rate $H=1.66 g_{*}{ }^{1 / 2}\left(T^{2} / M_{\mathrm{Pl}}\right)$, which gives a measure of the deviation from thermal equilibrium. For $K \ll 1$ one can find an approximate solution for Equation (108) given by

$$
n_{L}=\frac{s}{g_{*}}\left(\varepsilon_{v}+\varepsilon_{s}\right)
$$

The Yukawa couplings are constrained by the required amount of primordial lepton asymmetry required to generate the correct baryon asymmetry of the universe, while the lightest right handed neutrino mass is constrained from the out-ofequilibrium condition. In the resonant leptogenesis scenario, the $C P$ violation is largely enhanced, making the constrains on Yukawa couplings relaxed. Consequently the scale of leptogenesis can be considerably lower, making it possible to realize a $\mathrm{TeV}$ scale leptogenesis, which can be put to test at the LHC $[128,129]$.

\subsection{Leptogenesis With Triplet Higgs}

In section 2, we have discussed how small neutrino masses can be generated by adding triplet Higgs $\xi_{a}$ to the SM [22, 26-28, 130132]. The interactions of these triplet Higgs that are relevant for leptogenesis are given by

$$
\mathcal{L}_{\text {int }}=f_{i j} \xi \psi_{L i}=f_{i j}^{a} \xi_{a}^{++} l_{i} l_{j}+\mu_{a} \xi_{a}^{\dagger} \phi \phi .
$$

From these interactions we have the decay modes of the triplet Higgs

$$
\xi_{a}^{++} \rightarrow\left\{\begin{array}{c}
l_{i}^{+} l_{j}^{+} \\
\phi^{+} \phi^{+}
\end{array}\right.
$$

The $C P$ violation is obtained through the interference between the tree level and one-loop self-energy diagrams shown in Figure 10. There are no one-loop vertex diagrams in this case. One needs at least two $\xi$ 's. To see how this works, we will follow the mass-matrix formalism [22], in which the diagonal tree-level mass matrix of $\xi_{a}$ is modified in the presence of interactions to

$$
\frac{1}{2} \xi^{\dagger}\left(M_{+}^{2}\right)_{a b} \xi_{b}+\frac{1}{2}\left(\xi_{a}^{*}\right)^{\dagger}\left(M_{-}^{2}\right)_{a b} \xi_{b}^{*}
$$

where

$$
M_{ \pm}^{2}=\left(\begin{array}{cc}
M_{1}^{2}-i \Gamma_{11} M_{1} & -i \Gamma_{12}^{ \pm} \\
-i \Gamma_{21}^{ \pm} M_{1} & M_{2}^{2}-i \Gamma_{22} M_{2}
\end{array}\right),
$$

with $\Gamma_{a b}^{+}=\Gamma_{a b}$ and $\Gamma_{a b}^{-}=\Gamma_{a b}^{*}$. From the absorptive part of the one-loop diagram for $\xi_{a} \rightarrow \xi_{b}$ we obtain

$$
\Gamma_{a b} M_{b}=\frac{1}{8 \pi}\left(\mu_{a} \mu_{b}^{*}+M_{a} M_{b} \sum_{k, l} f_{k l}^{a *} f_{k l}^{b}\right) .
$$

Assuming $\Gamma_{a} \equiv \Gamma_{a a} \ll M_{a}$, the eigenvalues of $M_{ \pm}^{2}$ are given by

$$
\lambda_{1,2}=\frac{1}{2}\left(M_{1}^{2}+M_{2}^{2} \pm \sqrt{S}\right)
$$

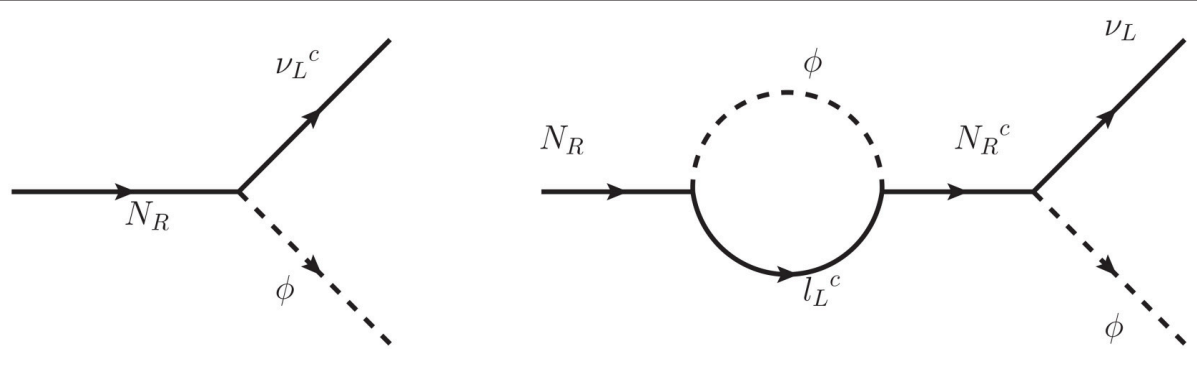

FIGURE 9 | Tree level and one-loop self-energy diagrams contributing to the CP violation in models with right handed neutrinos.
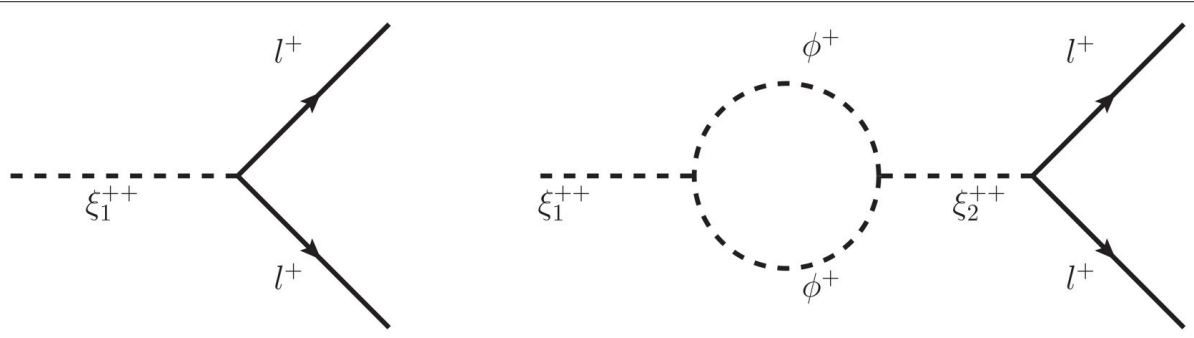

FIGURE 10 | Tree level and one-loop self-energy diagrams contributing to the CP violation in a model with triplet Higgs. 
where $S=\left(M_{1}^{2}-M_{2}^{2}\right)^{2}-4\left|\Gamma_{12} M_{2}\right|^{2}$ and $M_{1}>M_{2}$. The physical states, which evolves with time, can be written as linear combinations of the $C P$ eigenstates as

$$
\psi_{1,2}^{+}=a_{1,2}^{+} \xi_{1}+b_{1,2}^{+} \xi_{2}, \quad \psi_{1,2}^{-}=a_{1,2}^{-} \xi_{1}^{*}+b_{1,2}^{-} \xi_{2}^{*},
$$

where $a_{1}^{ \pm}=b_{2}^{ \pm}=1 / \sqrt{1+\left|C_{i}^{ \pm}\right|^{2}}, b_{1}^{ \pm}=C_{1}^{ \pm} / \sqrt{1+\left|C_{i}^{ \pm}\right|^{2}}$, $a_{2}^{ \pm}=C_{2}^{ \pm} / \sqrt{1+\left|C_{i}^{ \pm}\right|^{2}}$ with

$$
\begin{aligned}
& C_{1}^{+}=-C_{2}^{-}=\frac{-2 i \Gamma_{12}^{*} M_{2}}{M_{1}^{2}-M_{2}^{2}+\sqrt{S}}, \\
& C_{1}^{-}=-C_{2}^{+}=\frac{-2 i \Gamma_{12} M_{2}}{M_{1}^{2}-M_{2}^{2}+\sqrt{S}} .
\end{aligned}
$$

The physical states $\psi_{1,2}^{ \pm}$evolve with time and decay into lepton and antilepton pairs. Assuming $\left(M_{1}^{2}-M_{2}^{2}\right)^{2} \gg 4\left|\Gamma_{12} M_{2}\right|^{2}$, the $\mathrm{CP}$ asymmetry is given by $\mathrm{Ma}[22]$

$$
\varepsilon_{i} \simeq \frac{1}{8 \pi^{2}\left(M_{1}^{2}-M_{2}^{2}\right)^{2}} \sum_{k, l} \operatorname{Im}\left(\mu_{1} \mu_{2}^{*} f_{k l}^{1} f_{k l}^{2 *}\right)\left(\frac{M_{i}}{\Gamma_{i}}\right)
$$

For $M_{1}>M_{2}$, when the temperature drops below $M_{1}, \psi_{1}$ decays away to create a lepton asymmetry. However, this asymmetry is washed out by lepton number violating interactions of $\psi_{2}$; and the subsequent decay of $\psi_{2}$ at a temperature below $M_{2}$ sustains. The generated lepton asymmetry then gets converted to the baryon asymmetry in the presence of the sphaleron induced anomalous $B+L$ violating processes before the electroweak phase transition. The approximate final baryon asymmetry is given by

$$
\frac{n_{B}}{s} \sim \frac{\varepsilon_{2}}{3 g_{*} K(\ln K)^{0.6}},
$$

where $K \equiv \Gamma_{2}\left(T=M_{2}\right) / H\left(T=M_{2}\right)$ is the parameter measuring the deviation from thermal equilibrium when, $H=$ $1.66 g_{*}^{1 / 2}\left(T^{2} / M_{\mathrm{Pl}}\right)$ is the Hubble rate, and $g^{*}$ corresponds to the number of relativistic degrees of freedom.

In a more rigorous estimation of the baryon asymmetry, in addition to the decays and the inverse decays of triplet scalars, one needs to incorporate the gauge scatterings $\psi \bar{\psi} \leftrightarrow$ $F \bar{F}, \phi \bar{\phi}, G \bar{G}$ ( $F$ corresponds to SM fermions and $G$ corresponds to gauge bosons) and $\Delta L=2$ scattering processes $l l \leftrightarrow \phi^{*} \phi^{*}$ and $l \phi \leftrightarrow \bar{l} \phi^{*}$ into the Boltzmann equation analysis of the asymmetry. Including these washout processes, one finds a lower limit on $M_{\xi}, M_{\xi} \gtrsim 10^{11} \mathrm{GeV}$ [133]. For a quasi-degenerate spectrum of scalar triplets the resonance effect can enhance the $C P$ asymmetry by a large amount and a successful leptogenesis scenario can be attained for a much smaller value of triplet scalar mass. In Strumia [134] and Aristizabal Sierra et al. [135], an absolute bound of $M_{\xi} \gtrsim 1.6 \mathrm{TeV}$ is obtained for a successful resonant leptogenesis scenario with triplet Higgs.

\section{LEPTOGENESIS IN LRSM}

In Left-Right Symmetric Model (LRSM) [17, 19, 44-49] the leftright parity symmetry breaking implies the existence of a heavy right-handed charged gauge boson $W_{R}^{ \pm}$. In this section, we will discuss the aspect that if $W_{R}^{ \pm}$is detected at the LHC with a mass of a few $\mathrm{TeV}$ then it can have profound implications for leptogenesis. If indeed $W_{R}^{ \pm}$is detected at the LHC then that will give rise to an excess in the dilepton + dijet channel as reported sometime back by the CMS collaboration. A signal of $2.8 \sigma$ level was reported in the mass bin $1.8 \mathrm{TeV}<M_{l l j j}<$ $2.1 \mathrm{TeV}$ in the di-lepton + di-jet channel at the LHC by the CMS collaboration [136]. One of the popular interpretations of this signal was $W_{R}^{ \pm}$decay in the framework of LRSM with $g_{L} \neq g_{R}$ via an embedding of LRSM in $S O(10)[137,138]$. Another popular interpretation was for the case $g_{L}=g_{R}$ which utilized the CP phases and non-degenerate mass spectrum of the heavy neutrinos [139]. Around the same time the ATLAS collaboration had also reported a resonance signal decaying into a pair of SM gauge bosons. They found a local excess signal of $3.4 \sigma(2.5 \sigma$ global $)$ in the $W Z$ channel at around $2 \mathrm{TeV}$ [140]. This signal was shown to be explained by a $W_{R}$ in LRSM framework for a coupling $g_{R} \sim 0.4$ in Brehmer et al. [141]. Some other notable work along this direction can be found in Dobrescu and Liu Bhupal [142], Dev and Mohapatra [143] and Das et al. [144]. However, these interesting signals were either washed out by more accumulated data or reduced significantly below their initial reported levels. Nevertheless such signals have intrigued several studies concerning the impact of a TeV scale $W_{R}^{ \pm}$on leptogenesis.

As discussed earlier, the Higgs sector in one of the popular versions of LRSM consists of one bidoublet Higgs $\Phi$ and two triplet complex scalar fields $\Delta_{L, R}$. The relevant gauge transformations are as follows

$$
\Phi \sim(2,2,0,1), \quad \Delta_{L} \sim(3,1,2,1), \quad \Delta_{R} \sim(1,3,2,1) .
$$

Here one breaks the left-right symmetry in a spontaneous manner to reproduce the Standard Model. On the other hand the smallness of the neutrino masses is realized using the seesaw mechanism [14-20].

In another variant of LRSM one has only the doublet Higgs which are employed to break all the relevant symmetries. Here the Higgs sector consists of doublet scalars with the gauge transformations

$$
\Phi \sim(2,2,0,1), \quad H_{L} \sim(2,1,1,1), \quad H_{R} \sim(1,2,1,1)
$$

In addition there is one fermion gauge singlet $S_{R} \sim(\mathbf{1}, \mathbf{1}, \mathbf{0}, \mathbf{1})$. The Higgs doublet $H_{R}$ acquires a VEV to break the left-right symmetry which results in the mixing of $S$ with right-handed neutrinos. This gives rise to a light Majorana neutrino and a heavy pseudo-Dirac neutrino or alternatively a pair of Majorana neutrinos.

Historically, in LRSM, the left-right symmetry was broken at a fairly high scale, $M_{R}>10^{10} \mathrm{GeV}$. This serves two purposesfirstly, the requirement of gauge coupling unification implies this scale to be high, and secondly, thermal leptogenesis in this scenario gives a comparable bound. To get around this problem one often introduces a parity odd scalar which is then given a large VEV. This is often called $D$-parity breaking. 
Consequently, one can have $g_{L} \neq g_{R}$ even before the leftright symmetry breaking. This in turn allows the possibility of a gauge coupling unification with $\mathrm{TeV}$ scale $W_{R}$. This is true for both triplet and doublet models of LRSM. Embedding the LRSM in an $S O(10)$ GUT framework, the violation of $D$-parity [54] at a very high scale helps in explaining the CMS TeV scale $W_{R}$ signal for $g_{R} \approx 0.6 g_{L}$ as shown in Deppisch et al. [137, 138].

\subsection{Can a TeV-Scale $W_{R}^{ \pm}$at the LHC Falsify Leptogenesis?}

For a TeV scale $W_{R}^{ \pm}$, all leptogenesis scenarios may be broadly classified into two groups:

- At a very high scale a leptonic asymmetry is generated. It can be either in the context of LRSM with $D$-parity breaking or through some other interactions (both thermal and nonthermal).

- At the TeV scale a lepton asymmetry is generated with resonant enhancement, when the left-right symmetry breaking phase transition is taking place.

The following discussions hold for the LRSM variants with a Higgs sector consisting of triplet Higgs as well as a Higgs sector with exclusively doublet Higgs. We will often refer to these two broad classes of the LRSM mentioned above to discuss the lepton number violating washout processes and point out how all these possible scenarios of leptogenesis are falsifiable for a $W_{R}$ of $\mathrm{TeV}$ scale. In the case where high-scale leptogenesis happens at $T>10^{9} \mathrm{GeV}$, the low energy $B-L$ breaking gives rise to gauge interactions which depletes all the baryon asymmetry very rapidly before the electroweak phase transition is over. Now, these same lepton number violating gauge interactions will significantly slow down the generation of the lepton asymmetry for resonant leptogenesis at around $\mathrm{TeV}$ scale. Consequently, it is not possible to generate the required baryon asymmetry of the universe for TeV scale $W_{R}^{ \pm}$in this case.

For the case $M_{N_{3 R}} \gg M_{N_{2 R}} \gg M_{N_{1 R}}=M_{N_{R}}$, severe constraints on the $W_{R}^{ \pm}$mass for a successful scenario of highscale leptogenesis come from the $S U(2)_{R}$ gauge interactions as pointed out in Ma [145]. To have successful leptogenesis in the case $M_{N_{R}}>M_{W_{R}}$, the condition that the gauge scattering process $e_{R}^{-}+W_{R}^{+} \rightarrow N_{R} \rightarrow e_{R}^{+}+W_{R}^{-}$goes out-of-equilibrium yields

$$
M_{N_{R}} \gtrsim 10^{16} \mathrm{GeV}
$$

with $m_{W_{R}} / m_{N_{R}} \gtrsim 0.1$. For the scenario where $M_{W_{R}}>M_{N_{R}}$ leptogenesis happens either at $T>M_{W_{R}}$ after the breaking of $B-L$ gauge symmetry or at $T \simeq M_{N_{R}}$, the out-of equilibrium condition for the scattering process $e_{R}^{ \pm} e_{R}^{ \pm} \rightarrow W_{R}^{ \pm} W_{R}^{ \pm}$through $N_{R}$ exchange leads to the constraint

$$
M_{W_{R}} \gtrsim 3 \times 10^{6} \mathrm{GeV}\left(M_{N_{R}} / 10^{2} \mathrm{GeV}\right)^{2 / 3} .
$$

Thus, a $W_{R}$ with mass in the $\mathrm{TeV}$ range (in the case of a hierarchical neutrino mass spectrum) rules out the high-scale leptogenesis scenario. In Deppisch et al. [146, 147], neutrinoless double beta decay and the observation of the lepton number violating processes at the colliders were studied in the context of high-scale thermal leptogenesis. In Flanz et al. [117, 118], Pilaftsis [119], Roulet et al. [121], Buchmuller and Plumacher [122], Flanz and Paschos [123], Hambye et al. [124], Pilaftsis and Underwood [125] resonant leptogenesis has been discussed in the context of a considerably low mass $W_{R}$. In Frere et al. [148] it was pointed out that one requires an absolute lower bound of $18 \mathrm{TeV}$ on the $W_{R}$ mass in order to have successful low-scale leptogenesis with a quasi-degenerate right-handed neutrinos. Recently, it was found that just the correct lepton asymmetry can be obtained by utilizing relatively large Yukawa couplings, for $W_{R}$ mass scale higher than $13.1 \mathrm{TeV}$ in Bhupal Dev et al. $[149,150]$. Note that in Frere et al. [148] and Bhupal Dev et al. [150], the lepton number violating gauge scattering processes such as $N_{R} e_{R} \rightarrow \bar{u}_{R} d_{R}$, $N_{R} \bar{u}_{R} \rightarrow e_{R} d_{R}, N_{R} d_{R} \rightarrow e_{R} u_{R}$ and $N_{R} N_{R} \rightarrow e_{R} \bar{e}_{R}$ have been analyzed in detail. However, lepton number violating scattering processes with external $W_{R}$ were ignored because for a heavy $W_{R}$ there is a relative suppression of $e^{-m_{W_{R}} / m_{N_{R}}}$ in comparison to the processes where there is no $W_{R}$ in the external legs. Now, if indeed the mass of $W_{R}$ is around a few $\mathrm{TeV}$, as was suggested by an excess signal reported by the CMS experiment then one has to take the latter processes seriously. In Dhuria et al. [151], it was pointed out that the lepton number violating washout processes $\left(e_{R}^{ \pm} e_{R}^{ \pm} \rightarrow W_{R}^{ \pm} W_{R}^{ \pm}\right.$and $\left.e_{R}^{ \pm} W_{R}^{\mp} \rightarrow e_{R}^{\mp} W_{R}^{ \pm}\right)$can be mediated via the doubly charged Higgs in the conventional LRSM. In Bhupal Dev et al. [149] it was shown that in a parityasymmetric type-I seesaw model with relatively small $M_{N_{R}}$ one obtains a small contribution from this process which is expected for a large $M_{W_{R}} / M_{N_{R}}$. However, in this scenario some other relevant gauge scattering processes are efficient in washing out the lepton asymmetry. Including these washout processes one obtains a lower bound of $13.1 \mathrm{TeV}$ on the $W_{R}$ mass [149]. Here we will mainly discuss $\Delta_{R}^{++}$and $N_{R}$ mediated lepton number violating scattering processes in a much more general context to establish their importance as washout processes which can falsify the possibility of leptogenesis depending on $W_{R}$ mass [152]. One of the vertices in the $\Delta_{R}^{++}$mediated process is gauge vertex while the other one is a Yukawa vertex. On the other hand for $N_{R}$ mediated lepton number violating scattering processes both the vertices are gauge vertices. Consequently, these lepton number violating scattering processes are very rapid as compared to the scattering processes involving only Yukawa vertices. It turns out that $N_{R}$ and $\Delta_{R}^{++}$mediated scattering process $e_{R}^{ \pm} W_{R}^{\mp} \rightarrow$ $e_{R}^{\mp} W_{R}^{ \pm}$does not go out of equilibrium till the electroweak phase transition if the mass of $W_{R}$ is around $\mathrm{TeV}$ scale. Consequently, these lepton number violating scattering processes continue to wash out or slow down the generation of lepton asymmetry ${ }^{8}$. In the scenario of LRSM involving only doublet Higgs in the Higgs sector the doubly charged Higgs is absent. Nevertheless, the $N_{R}$ mediated lepton number violating scattering processes will be present and will wash out the lepton asymmetry in such a scenario.

\footnotetext{
${ }^{8}$ In passing we would like to note that the other relevant lepton number violating scattering process is doubly phase space suppressed for a temperature below the $W_{R}$ mass scale. Consequently, we will neglect such a process for leptogenesis occurring at $T \lesssim M_{W_{R}}$.
} 
In LRSM the right handed leptonic charged current interaction is given by

$$
\mathcal{L}_{N}=\frac{1}{2 \sqrt{2}} g_{R} J_{R \mu} W_{R}^{-\mu}+\text { h.c. }
$$

where $J_{R \mu}=\bar{e}_{R} \gamma_{\mu}\left(1+\gamma_{5}\right) N_{R}$. The relevant interactions of the right-handed Higgs triplet are given by

$$
\mathcal{L}_{\Delta_{R}} \supset\left(D_{R \mu} \vec{\Delta}_{R}\right)^{\dagger}\left(D_{R}^{\mu} \vec{\Delta}_{R}\right)
$$

where $\vec{\Delta}_{R}=\left(\Delta_{R}^{++}, \Delta_{R}^{+}, \Delta_{R}^{0}\right)$. The covariant derivative is given by $D_{R \mu}=\partial_{\mu}-i g_{R}\left(T_{R}^{j} A_{R \mu}^{j}\right)-i g^{\prime} B_{\mu}$, where $A_{R \mu}^{j}$ and $B_{\mu}$ are gauge fields corresponding to $S U(2)_{R}$ and $U(1)_{B-L}$ gauge groups with the associated gauge couplings given by $g_{R}$ and $g^{\prime}$, respectively. When the neutral Higgs field $\Delta_{R}^{0}$ acquires a $\operatorname{VEV}\left\langle\Delta_{R}^{0}\right\rangle=\frac{1}{\sqrt{2}} v_{R}$ $S U(2)_{R}$, the interaction between the gauge boson $W_{R}$ and the doubly charged Higgs is given by Doi [153]

$$
\mathcal{L}_{\Delta_{R}} \supset\left(-\frac{v_{R}}{\sqrt{2}}\right) g_{R}^{2} W_{\mu R}^{-} W_{R}^{-\mu} \Delta_{R}^{++}+\text {h.c. }
$$

The Yukawa interaction between the lepton doublet $\psi_{e R}=$ $\left(N_{R}, e_{R}\right)^{T}$ and the components of triplet Higgs $\vec{\Delta}_{R}$ are given by

$$
\mathcal{L}_{Y}=h_{e e}^{R} \overline{\left(\psi_{e R}\right)^{c}}\left(i \tau_{2} \vec{\tau} \cdot \vec{\Delta}_{R}\right) \psi_{e R}+\text { h.c. }
$$

where $\tau$ 's are the Pauli matrices. After the Higgs triplet field acquires a VEV, the relevant Yukawa coupling can be written as $h_{e e}^{R}=\frac{M_{N_{R}}}{2 v_{R}}$ where $M_{N_{R}}$ is the Majorana mass of $N_{R}$.

The relevant Feynman diagrams for the lepton number violating processes induced by these interactions are depicted in Figure 11.

Using the interactions given in Equations (126)-(129), one can estimate the differential scattering cross section for the process $e_{R}^{\mp}(p) W_{R}^{ \pm}(k) \rightarrow e_{R}^{ \pm}\left(p^{\prime}\right) W_{R}^{\mp}\left(k^{\prime}\right)$ to obtain [153]

$$
\frac{d \sigma_{e_{R} W_{R}}^{e_{R} W_{R}}}{d t}=\frac{1}{384 \pi M_{W_{R}}^{4}\left(s-M_{W_{R}}^{2}\right)^{2}} \Lambda_{e_{R} W_{R}}^{e_{R} W_{R}}(s, t, u),
$$

where

$$
\Lambda_{e_{R} W_{R}}^{e_{R} W_{R}}(s, t, u)=\left.\Lambda_{e_{R} W_{R}}^{e_{R} W_{R}}(s, t, u)\right|_{N_{R}}+\left.\Lambda_{e_{R} W_{R}}^{e_{R} W_{R}}(s, t, u)\right|_{\Delta_{R}^{++}}
$$

and

$$
\begin{aligned}
& \left.\Lambda_{e_{R} W_{R}}^{e_{R} W_{R}}(s, t, u)\right|_{N_{R}}=g_{R}^{4}\left\{-t\left|M_{N_{R}}\left(\frac{s}{s-M_{N_{R}}^{2}}+\frac{u}{u-M_{N_{R}}^{2}}\right)\right|^{2}\right. \\
& -4 M_{W_{R}}^{2}\left(s u-M_{W_{R}}^{4}\right)(s-u)^{2}\left|\frac{M_{N_{R}}}{\left(s-M_{N_{R}}^{2}\right)\left(u-M_{N_{R}}^{2}\right)}\right|^{2} \\
& \left.-4 M_{W_{R}}^{4} t\left(\left|\frac{m_{N_{R}}}{\left(s-M_{N_{R}}^{2}\right)}\right|^{2}+\left|\frac{M_{N_{R}}}{\left(u-M_{N_{R}}^{2}\right)}\right|^{2}\right)\right\}
\end{aligned}
$$

$$
\begin{aligned}
& \left.\Lambda_{e_{R} W_{R}}^{e_{R} W_{R}}(s, t, u)\right|_{\Delta_{R}^{++}} ^{+\infty}=4 g_{R}^{4}(-t)\left\{\frac{(s+u)^{2}+8 M_{W_{R}}^{4}}{\left(t-M_{\Delta_{R}}^{2}\right)^{2}}\left|M_{N_{R}}\right|^{2}\right. \\
& \quad+\frac{(s+u)}{t-M_{\Delta_{R}}^{2}}\left|M_{N_{R}}\right|^{2}\left(\frac{s}{s-M_{N_{R}}^{2}}+\frac{u}{u-M_{N_{R}}^{2}}\right) \\
& \left.+\frac{4 M_{W_{R}}^{4}}{t-M_{\Delta_{R}}^{2}}\left|M_{N_{R}}\right|^{2}\left(\frac{1}{s-M_{N_{R}}^{2}}+\frac{1}{u-M_{N_{R}}^{2}}\right)\right\}
\end{aligned}
$$

where we neglect any mixing between $W_{L}$ and $W_{R}$. On the righthand side of Equation (133), the first term corresponds to the Higgs exchange. The last two terms are due to the interference between Higgs and $N_{R}$ exchange. The Mandelstam variables $s=$ $(p+k)^{2}, t=\left(p-p^{\prime}\right)^{2}$ and $u=\left(p-k^{\prime}\right)^{2}$ are related by the scattering angle $\theta$ as follows

$$
\left(\begin{array}{c}
s t \\
s u-M_{W_{R}}^{4}
\end{array}\right)=-\frac{1}{2}\left(s-M_{W_{R}}^{2}\right)^{2}(1 \mp \cos \theta) .
$$

The differential scattering cross section for the process $e_{R}^{ \pm}(p) e_{R}^{ \pm}\left(p^{\prime}\right) \rightarrow W_{R}^{ \pm}(k) W_{R}^{ \pm}\left(k^{\prime}\right)$ is given by Doi [153]

$$
\frac{d \sigma_{W_{R} W_{R}}^{e_{R} e_{R}}}{d t}=\frac{1}{512 \pi M_{W_{R}}^{4} s^{2}} \Lambda_{W_{R} W_{R}}^{e_{R} e_{R}}(s, t, u),
$$

where

$$
\Lambda_{W_{R} W_{R}}^{e_{R} e_{R}}(s, t, u)=\left.\Lambda_{W_{R} W_{R}}^{e_{R} e_{R}}(s, t, u)\right|_{N_{R}}+\left.\Lambda_{W_{R} W_{R}}^{e_{R} e_{R}}(s, t, u)\right|_{\Delta_{R}^{++}} .
$$

The expressions for $\Lambda_{W_{R} W_{R}}^{e_{R} e_{R}}(s, t, u)$ can be obtained after interchanging $s \leftrightarrow t$ in $\Lambda_{e_{R} W_{R}}^{e_{R} W_{R}}(s, t, u): \Lambda_{W_{R} W_{R}}^{e_{R} e_{R}}(t, s, u)=$ $-\Lambda_{e_{R} W_{R}}^{e_{R} W_{R}}(s, t, u)$. The Mandelstem variables $t=(p-k)^{2}$ and $u=\left(p-k^{\prime}\right)^{2}$ can be written in terms of $s=\left(p+p^{\prime}\right)^{2}$ and scattering angle $\theta$ as follows

$\left(\begin{array}{c}t \\ u\end{array}\right)=-\frac{s}{2}\left(1-\frac{2 M_{W_{R}}^{2}}{s}\right)\left\{1 \mp \sqrt{1-\left(\frac{2 M_{W_{R}}^{2}}{s-2 M_{W_{R}}^{2}}\right)^{2}} \cos \theta\right\}$.

\subsubsection{Wash Out of Lepton Asymmetry for $T>M_{W_{R}}$}

During the period when the temperature is such that $v_{R}>T>$ $M_{W_{R}}$, the lepton number violating washout processes are very rapid in the absence any suppression. To have a quantitative estimate of the strength of these scattering processes in depleting the lepton asymmetry one can estimate the parameter defined as

$$
K \equiv \frac{n\langle\sigma|v|\rangle}{H}
$$

for both the processes during $v_{R}>T>M_{W_{R}}$, where $n$ corresponds to the number density of relativistic species and is given by $n=2 \times \frac{3 \zeta(3)}{4 \pi^{2}} T^{3}$. $H$ corresponds to the Hubble 


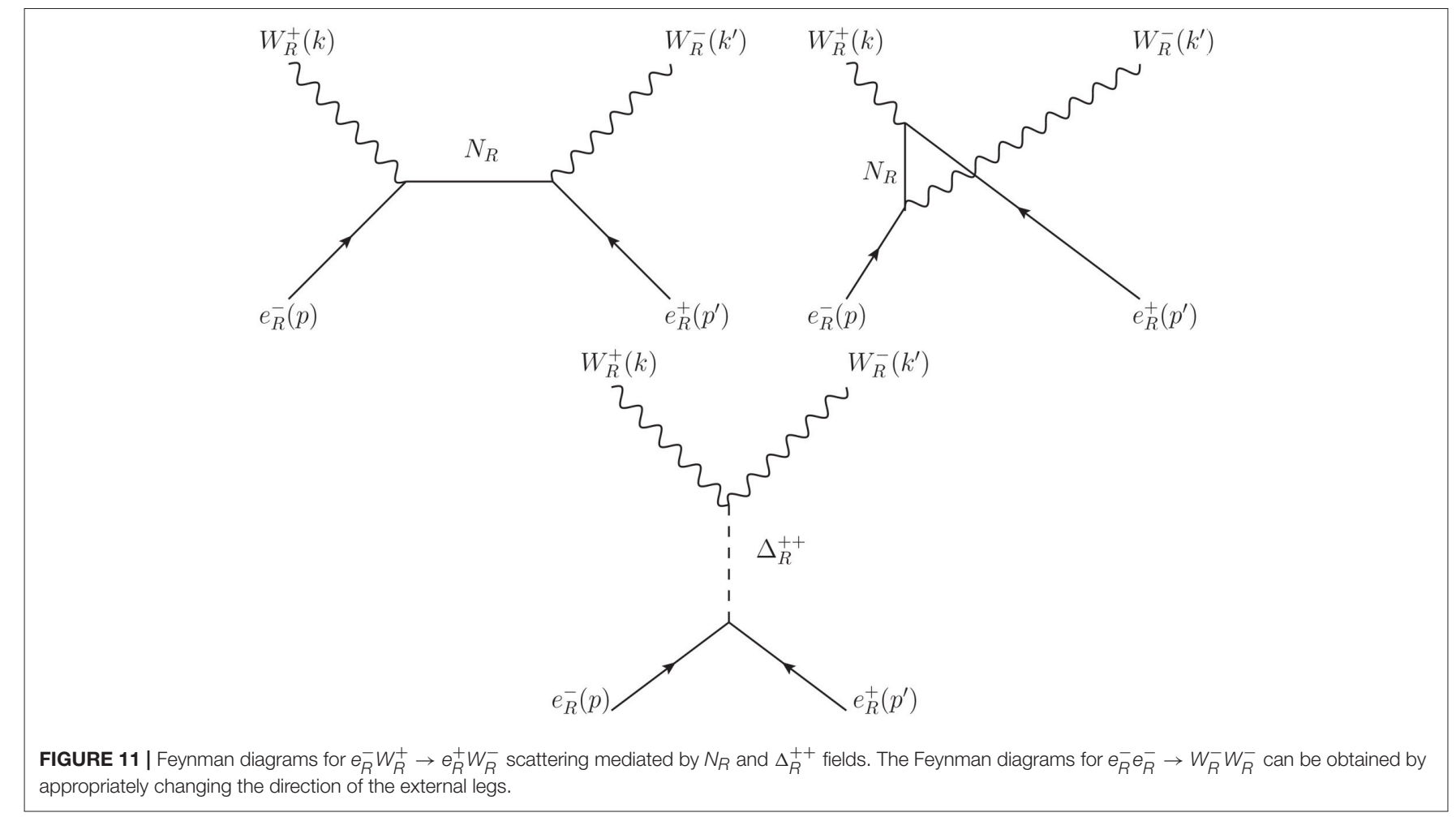

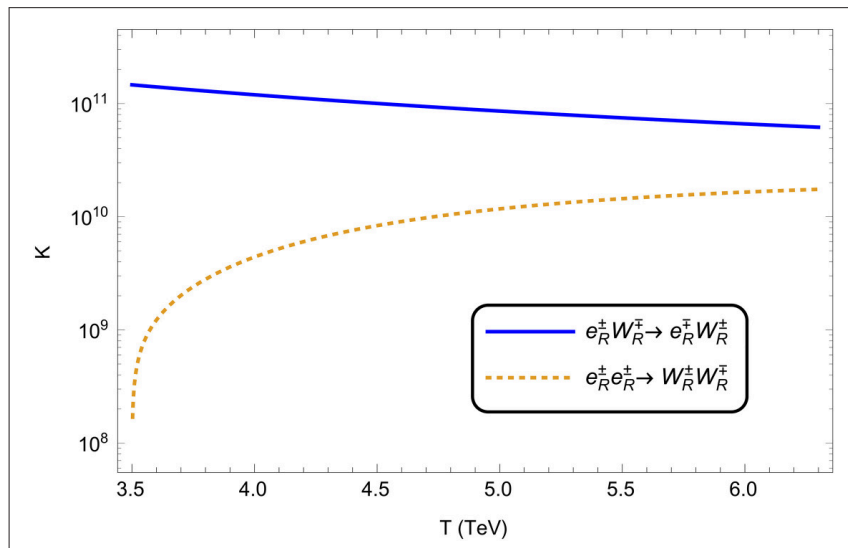

FIGURE 12 | Plot showing the behavior of $K$ as a function of temperature $T$ for the processes $e_{R}^{ \pm} W_{R}^{\mp} \rightarrow e_{R}^{\mp} W_{R}^{ \pm}$and $e_{R}^{ \pm} e_{R}^{ \pm} \rightarrow W_{R}^{ \pm} W_{R}^{ \pm}$(including both $\Delta_{R}^{++}$and $N_{R}$ mediated diagrams) for $v_{R}>T>M_{W_{R}}$. The right handed charged gauge boson mass is taken to be $M_{W_{R}}=3.5 \mathrm{TeV}$.

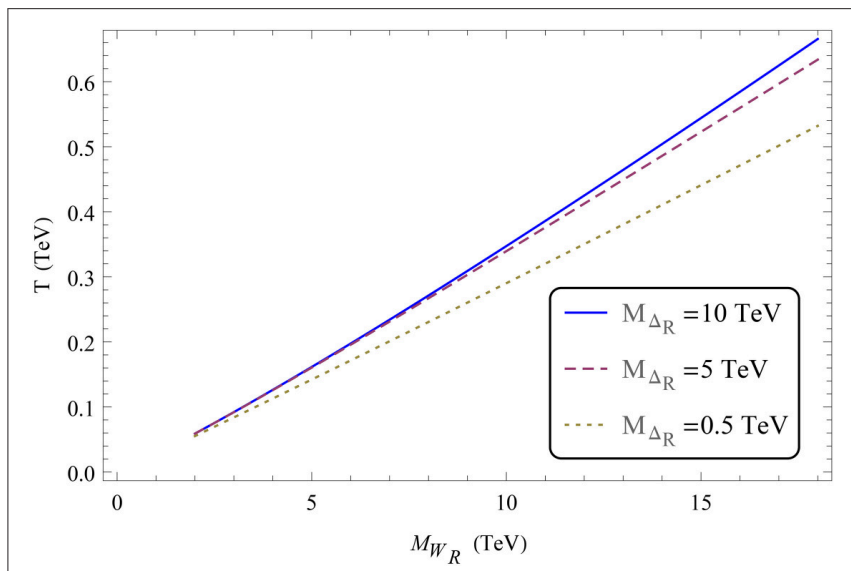

FIGURE 13 | Plot showing the dependance of out of equilibrium temperature $(T)$ on $M_{W_{R}}$ for the process $e_{R}^{ \pm} W_{R}^{\mp} \rightarrow e_{R}^{\mp} W_{R}^{ \pm}$(mediated via $\Delta_{R}^{++}$and $N_{R}$ fields) for $M_{N_{R}} \sim M_{W_{R}}$. The three different lines correspond to three different values of $M_{\Delta_{R}}$. rate $H \simeq 1.7 g_{*}^{1 / 2} T^{2} / M_{\mathrm{Pl}}$, where $g_{*} \sim 100$ corresponds to the relativistic degrees of freedom. The thermally averaged cross section is denoted by $\langle\sigma|v|\rangle$. To choose a rough estimate of $v_{R}$, let us compare the situation with the Standard Model, where we have $\langle\phi\rangle=\frac{v_{L}}{\sqrt{2}}$ where $v_{L}=246 \mathrm{GeV}$, and $M_{W_{L}} \sim 80 \mathrm{GeV}$. Now in case of LRSM $\left\langle\Delta_{R}^{0}\right\rangle=\frac{v_{R}}{\sqrt{2}}$ breaks the left-right symmetry and $M_{W_{R}}=g_{R} v_{R}$. Taking $g_{R} \sim g_{L}$, we have $\frac{\langle\phi\rangle}{M_{W_{L}}}=\frac{\left\langle\Delta_{R}^{0}\right\rangle}{M_{W_{R}}} \approx 3$.

Making use of the differential scattering cross sections in Equations (130) and (135), we plot the behavior of $K$ as a function of temperature in Figure 12. The plot corresponds to a temperature range $3 M_{W_{R}}>T>M_{W_{R}}$ and right handed charged gauge boson mass $M_{W_{R}}=3.5 \mathrm{TeV}$.

In Figure 12, the large values of $K$ for both the processes indicates that high wash out efficiency of these scattering processes for $T \gtrsim M_{W_{R}}$. For the LRSM variant with its Higgs sector consisting of only doublet Higgs the doubly charged Higgs mediated channels are absent for these processes and the 
right handed neutrino will mediate these processes, which will washout the lepton asymmetry for $T \gtrsim M_{W_{R}}$.

\subsubsection{Wash Out of Asymmetry for $T<M_{W_{R}}$}

During the period when the temperature is such that $T<M_{W_{R}}$, the process $e_{R}^{ \pm} W_{R}^{\mp} \rightarrow e_{R}^{\mp} W_{R}^{ \pm}$is of more importance. Let us now estimate a lower bound on $T$ below $T=M_{W_{R}}$ till which this process stays in equilibrium and continues to deplete lepton asymmetry. The scattering rate can be written as ${ }^{9} \Gamma=\bar{n}\left\langle\sigma v_{\text {rel }}\right\rangle$. For $T<M_{W_{R}}$ the Boltzmann suppression of the scattering rate stems from the number density $\bar{n}=g\left(\frac{T M_{W_{R}}}{2 \pi}\right)^{3 / 2} \exp \left(-\frac{M_{W_{R}}}{T}\right)$. Now, the scattering process stays in thermal equilibrium when the condition $\Gamma>H$ is satisfied.

In Figure 13 we show the temperature until which the scattering process $e_{R}^{ \pm} W_{R}^{\mp} \rightarrow e_{R}^{\mp} W_{R}^{ \pm}$stays in equilibrium as a function of $M_{W_{R}}$ for three values of $M_{\Delta_{R}}$ and taking $M_{N_{R}} \lesssim M_{W_{R}}$ and $v_{\text {rel }}=1$. We have taken the lowest value of $M_{\Delta_{R}}$ to be 500 $\mathrm{GeV}$ to be consistent with the recent collider limits on the doubly charged Higgs mass [154]. The plot shows that unless $M_{W_{R}}$ is significantly heavier than a few $\mathrm{TeV}$, the process $e_{R}^{ \pm} W_{R}^{\mp} \rightarrow$ $e_{R}^{\mp} W_{R}^{ \pm}$will continue to be in equilibrium till a temperature similar to the electroweak phase transition. Consequently, this process will continue to washout or slow down the generation of lepton asymmetry until the electroweak phase transition. In the LRSM variant with doublet Higgs, the heavy neutrinos will mediated lepton number violating scattering processes will washout or slow down the generation of lepton asymmetry until the electroweak phase transition. Thus, the lower limit on the $W_{R}$ mass for a successful leptogenesis scenario is significantly higher a few $\mathrm{TeV}$. This was also confirmed by explicitly solving the relevant Boltzmann equations in Bhupal Dev et al. $[149,150]$.

\section{CONCLUDING REMARKS}

We have reviewed the standard left-right symmetric theories and the implementation of different types of low scale seesaw mechanisms in the context of neutrino masses. We have also discussed a left-right symmetric model with additional vectorlike fermions in order to simultaneously explain the charged fermion and Majorana neutrino masses. In this model the quark and charged lepton masses and mixings are realized via a universal seesaw mechanism while spontaneous symmetry breaking is achieved with two doublet Higgs fields with non-zero

\footnotetext{
${ }^{9}$ We ignore any finite temperature effects to simplify the analysis.
}

\section{REFERENCES}

1. Keung WY, Senjanovic G. Majorana neutrinos and the production of the right-handed charged gauge boson. Phys Rev Lett. (1983) 50:1427. doi: 10.1103/PhysRevLett.50.1427

2. Nemevsek M, Nesti F, Senjanovic G, Zhang Y. First limits on leftright symmetry scale from LHC data. Phys Rev D (2011) 83:115014. arXiv:1103.1627. doi: 10.1103/PhysRevD.83.115014
$B-L$ charge, we have introduced scalar triplets with small induced VEVs such that they give Majorana masses to light as well as heavy neutrinos. We have also discussed how the Majorana nature of these neutrinos leads to $0 v \beta \beta$ decay. Interestingly, the right-handed currents play an important role in discriminating between the mass hierarchy as well as the absolute scale of light neutrinos. To summarize the situation for leptogenesis, in the high-scale leptogenesis scenario $\left(T \gtrsim M_{W_{R}}\right)$, in all the variants of LRSM the lepton number violating processes $e_{R}^{ \pm} e_{R}^{ \pm} \rightarrow W_{R}^{ \pm} W_{R}^{ \pm}$and $e_{R}^{ \pm} W_{R}^{\mp} \rightarrow e_{R}^{\mp} W_{R}^{ \pm}$are highly efficient in washing out the lepton asymmetry. In the case of resonant leptogenesis scenario at around $\mathrm{TeV}$ scale we found that the latter process stays in equilibrium until the electroweak phase transition, making the generation of lepton asymmetry for $T<$ $M_{W_{R}}$ significantly weaker. Thus, if the LHC discovers a TeV scale $W_{R}^{ \pm}$then one needs to look for some post-electroweak phase transition mechanism to explain the baryon asymmetry of the Universe. To this end the observation of the neutronantineutron oscillation $[155,156]$ or $(B-L)$ violating proton decay [157] will play a guiding role in confirming such scenarios. Complementing these results, the low-energy subgroups of the superstring motivated $E_{6}$ model have also been explored which can also give rise to left-right symmetric gauge structures but with a number of additional exotic particles as compared to the conventional LRSM. Interestingly, one of the low-energy supersymmetric subgroups of $E_{6}$, also known as the Alternative Left-Right Symmetric Model, gives a model alternative to successfully realize high-scale leptogenesis in the absence of the dangerous gauge washout processes [158]. The vector-like fermions added to the minimal framework of LRSM to realize a universal seesaw can pave new ways to realize baryogenesis as discussed in Deppisch et al. [73].

\section{AUTHOR CONTRIBUTIONS}

All authors listed have made a substantial, direct and intellectual contribution to the work, and approved it for publication.

\section{ACKNOWLEDGMENTS}

The authors would like to thank Frank F. Deppisch for many helpful discussions and encouragement. The authors are also thankful to both the reviewers for many valuable suggestions and $\mathrm{CH}$ and US are thankful to Mansi Dhuria and Raghavan Rangarajan for several wonderful collaborations which have resulted in some of the interesting findings covered in this review. The work of US is supported partly by the JC Bose National Fellowship grant under DST, India.

3. Das SP, Deppisch FF, Kittel O, Valle JWF. Heavy neutrinos and lepton flavour violation in left-right symmetric models at the LHC. Phys Rev D (2012) 86:055006. 055006, arXiv:1206.0256. doi: 10.1103/PhysRevD.86.0 55006

4. Chen CY, Dev PSB, Mohapatra RN. Probing heavy-light neutrino mixing in left-right seesaw models at the LHC. Phys Rev D (2013) 88:033014. arXiv:1306.2342. doi: 10.1103/PhysRevD.88.0 33014 
5. Mitra M, Ruiz R, Scott DJ, Spannowsky M. Neutrino jets from high-mass $W_{R}$ gauge bosons in TeV-scale left-right symmetric models. Phys Rev D (2016) 94:095016. arXiv:1607.03504. doi: 10.1103/PhysRevD.94.095016

6. Bambhaniya G, Chakrabortty J, Gluza J, Jelinski T, Szafron R. Search for doubly charged Higgs bosons through vector boson fusion at the LHC and beyond. Phys Rev D (2015) 92:015016. arXiv:1504.03999.

7. Dutta B, Eusebi R, Gao Y, Ghosh T, Kamon T. Exploring the doubly charged Higgs boson of the left-right symmetric model using vector boson fusionlike events at the LHC. Phys Rev D (2014) 90:055015. arXiv:1404.0685. doi: 10 . 1103/PhysRevD.90.055015

8. Dev PSB, Mohapatra RN, Zhang Y. Probing the higgs sector of the minimal left-right symmetric model at future hadron colliders. JHEP (2016) 05:174. doi: 10.1007/JHEP05(2016)174

9. Mitra M, Niyogi S, Spannowsky M. Type-II seesaw model and multilepton signatures at hadron colliders. Phys Rev D (2017) 95:035042. doi: 10.1103/PhysRevD.95.035042

10. Weinberg S. Baryon and lepton nonconserving processes. Phys Rev Lett. (1979) 43:1566-70. doi: 10.1103/PhysRevLett.43.1566

11. Wilczek F, Zee A. Operator analysis of nucleon decay. Phys Rev Lett. (1979) 43:1571-3. doi: 10.1103/PhysRevLett.43.1571

12. Weinberg S. Varieties of baryon and lepton nonconservation. Phys Rev D (1980) 22:1694. doi: 10.1103/PhysRevD.22.1694

13. Weldon HA, Zee A. Operator analysis of new physics. Nucl Phys B (1980) 173:269-90. doi: 10.1016/0550-3213(80)90218-7

14. Minkowski P. $\mu \rightarrow e \gamma$ at a Rate of One Out of $10^{9}$ Muon Decays? Phys Lett $B$ (1977) 67:421-8.

15. Gell-Mann M, Ramond P, Slansky R. Complex spinors and unified theories. Conf Proc. (1979) C790927:315-21.

16. Yanagida T. Horizontal symmetry and masses of neutrinos. Conf Proc. (1979) C7902131:95-9.

17. Mohapatra RN, Senjanović G. Neutrino mass and spontaneous parity violation. Phys Rev Lett. (1980) 44:912. doi: 10.1103/PhysRevLett.44.912

18. Schechter J, Valle JWF. Neutrino masses in SU(2) x U(1) theories. Phys Rev $D$ (1980) 22:2227. doi: 10.1103/PhysRevD.22.2227

19. Mohapatra RN, Senjanovic G. Neutrino masses and mixings in gauge models with spontaneous parity violation. Phys Rev D (1981) 23:165. doi: 10.1103/PhysRevD.23.165

20. Schechter J, Valle JWF. Neutrino decay and spontaneous violation of lepton number. Phys Rev D (1982) 25:774. doi: 10.1103/PhysRevD.25.774

21. Fukugita M, Yanagida T. Baryogenesis without grand unification. Phys Lett $B$ (1986) 174:45-7. doi: 10.1016/0370-2693(86)91126-3

22. Ma E, Sarkar U. Neutrino masses and leptogenesis with heavy Higgs triplets. Phys Rev Lett. (1998) 80:5716-9. doi: 10.1103/PhysRevLett.80.5716

23. Magg M, Wetterich C. Neutrino mass problem and gauge hierarchy. Phys Lett B (1980) 94:61-4. doi: 10.1016/0370-2693(80)90825-4

24. Cheng TP, Li LF. Neutrino masses, mixings and oscillations in SU(2) x U(1) models of electroweak interactions. Phys Rev D (1980) 22:2860. doi: 10.1103/PhysRevD.22.2860

25. Lazarides G, Shafi Q, Wetterich C. Proton lifetime and fermion masses in an SO(10) model. Nucl Phys B (1981) 181:287-300.

26. Lazarides G, Shafi Q. R symmetry in minimal supersymmetry standard model and beyond with several consequences. Phys Rev D (1998) 58:071702.

27. Grimus W, Pfeiffer R, Schwetz T. A Four neutrino model with a Higgs triplet. Eur Phys J C (2000) 13:125-32. doi: 10.1007/s100520000255

28. Hambye T, Ma E, Sarkar U. Supersymmetric triplet Higgs model of neutrino masses and leptogenesis. Nucl Phys B (2001) 602:23-38. doi: 10.1016/S0550-3213(01)00109-2

29. Foot R, Lew H, He XG, Joshi GC. Seesaw neutrino masses induced by a triplet of leptons. Z Phys C (1989) 44:441.

30. Ma E. Pathways to naturally small neutrino masses. Phys Rev Lett. (1998) 81:1171-4. doi: 10.1103/PhysRevLett.81.1171

31. Zee A. A theory of lepton number violation, neutrino majorana mass, and oscillation. Phys Lett B (1980) 93:389. doi: 10.1016/0370-2693(80)90349-4 [Erratum: Phys. Lett.B95,461(1980)].

32. Wolfenstein L. A theoretical pattern for neutrino oscillations. Nucl Phys B (1980) 175:93-6. doi: 10.1016/0550-3213(80)90004-8

33. Zee A. Quantum numbers of majorana neutrino masses. Nucl Phys B (1986) 264:99-110.
34. Babu KS. Model of “calculable” majorana neutrino masses. Phys Lett B (1988) 203:132-6.

35. Ma E. Verifiable radiative seesaw mechanism of neutrino mass and dark matter. Phys Rev D (2006) 73:077301. arXiv:hep-ph/0601225. doi: 10.1103/ PhysRevD.73.077301

36. Krauss LM, Nasri S, Trodden M. A model for neutrino masses and dark matter. Phys Rev D (2003) 67:085002. arXiv:hep-ph/0210389. doi: 10.1103/ PhysRevD.67.085002

37. Aoki M, Kanemura S, Seto O. Neutrino mass, dark matter and baryon asymmetry via TeV-scale physics without fine-tuning. Phys Rev Lett. (2009) 102:051805. doi: 10.1103/PhysRevLett.102.051805

38. Gustafsson M, No JM, Rivera MA. Predictive model for radiatively induced neutrino masses and mixings with dark matter. Phys Rev Lett. (2013) 110:211802. arXiv:1212.4806. doi: 10.1103/PhysRevLett.110.211802 [Erratum: Phys. Rev. Lett.112: 259902(2014)].

39. Kanemura S, Sugiyama H. Dark matter and a suppression mechanism for neutrino masses in the Higgs triplet model. Phys Rev D (2012) 86:073006. doi: 10.1103/PhysRevD.86.073006

40. Nasri S, Moussa S. Model for small neutrino masses at the TeV scale. Mod Phys Lett A (2002) 17:771-8. doi: 10.1142/S02177323020 07119

41. Gu PH, Sarkar U. Radiative neutrino mass, dark matter and leptogenesis. Phys Rev D (2008) 77:105031. doi: 10.1103/PhysRevD.77.1 05031

42. Kanemura S, Matsui T, Sugiyama H. Loop suppression of dirac neutrino mass in the neutrinophilic two higgs doublet model. Phys Lett B (2013) 727:151-6. arXiv:1305.4521. doi: 10.1016/j.physletb.2013.09.061

43. Bonilla C, Ma E, Peinado E, Valle JWF. Two-loop Dirac neutrino mass and WIMP dark matter. Phys Lett B (2016) 762:214-8. arXiv:1607.03931.

44. Pati JC, Salam A. Is baryon number conserved? Phys Rev Lett. (1973) 31:661-4. doi: 10.1103/PhysRevLett.31.661

45. Pati JC, Salam A. Unified lepton-hadron symmetry and a gauge theory of the basic interactions. Phys Rev D (1973) 8:1240-51. doi: 10.1103/PhysRevD.8.1240

46. Pati JC, Salam A. Lepton number as the fourth color. Phys Rev D (1974) 10:275-89.

47. Mohapatra RN, Pati JC. A natural left-right symmetry. Phys Rev D (1975) 11:2558.

48. Senjanović G, Mohapatra RN. Exact left-right symmetry and spontaneous violation of parity. Phys Rev D (1975) 12:1502. doi: 10.1103/PhysRevD.12.1502

49. Mohapatra RN, Marshak RE. Local B-L symmetry of electroweak interactions, majorana neutrinos and neutron oscillations. Phys Rev Lett. (1980) 44:1316-9. [Erratum: Phys. Rev. Lett.44: 1643(1980)]. doi: 10.1103/PhysRevLett.44.1316

50. Mohapatra RN. Mechanism for understanding small neutrino mass in superstring theories. Phys Rev Lett. (1986) 56:561-3. doi: 10.1103/PhysRevLett.56.561

51. Mohapatra RN, Valle JWF. Neutrino mass and baryon number nonconservation in superstring models. Phys Rev D (1986) 34:1642.

52. Akhmedov EK, Lindner M, Schnapka E, Valle JWF. Left-right symmetry breaking in NJL approach. Phys Lett B (1996) 368:270-80.

53. Malinsky M, Romao JC, Valle JWF. Novel supersymmetric SO(10) seesaw mechanism. Phys Rev Lett. (2005) 95:161801. doi: 10.1103/PhysRevLett.95.161801

54. Chang D, Mohapatra RN, Parida MK. Decoupling parity and SU(2)-R breaking scales: a new approach to left-right symmetric models. Phys Rev Lett. (1984) 52:1072. doi: 10.1103/PhysRevLett.52.1072

55. Gavela MB, Hambye T, Hernandez D, Hernandez P. Minimal flavour seesaw models. JHEP (2009) 09:038. arXiv:0906.1461.

56. Barry J, Rodejohann W, Zhang H. Light sterile neutrinos: models and phenomenology. JHEP (2011) 07:091.arXiv:1105.3911. doi: 10.1007/ JHEP07(2011)091

57. Zhang H. Light sterile neutrino in the minimal extended seesaw. Phys Lett $B$ (2012) 714:262-6. doi: 10.1016/j.physletb.2012.06.074

58. Dev PSB, Pilaftsis A. Minimal radiative neutrino mass mechanism for inverse seesaw models. Phys Rev D (2012) 86:113001. doi: 10.1103/PhysRevD.86.113001 
59. Dev PSB, Mohapatra RN. TeV scale inverse seesaw in $\mathrm{SO}(10)$ and leptonic non-unitarity effects. Phys Rev D (2010) 81:013001. arXiv:0910.3924. doi: 10. 1103/PhysRevD.81.013001

60. Awasthi RL, Parida MK, Patra S. Neutrino masses, dominant neutrinoless double beta decay, and observable lepton flavor violation in left-right models and $\mathrm{SO}(10)$ grand unification with low mass $W_{R}, Z_{R}$ bosons. JHEP (2013) 1308:122. doi: 10.1007/JHEP08(2013)122

61. Deppisch FF, Graf L, Kulkarni S, Patra S, Rodejohann W, Sahu N, et al. Reconciling the $2 \mathrm{TeV}$ excesses at the LHC in a linear seesaw leftright model. Phys Rev D (2016) 93:013011. doi: 10.1103/PhysRevD.93. 013011

62. Davidson A, Wali KC. Universal seesaw mechanism? Phys Rev Lett. (1987) 59:393. doi: 10.1103/PhysRevLett.59.393

63. Rajpoot S. Seesaw masses for quarks and leptons in an ambidextrous electroweak interaction model. Phys Lett B (1987) 191:122-6. doi: 10.1016/0370-2693(87)91332-3

64. Chang D, Mohapatra RN. Small and calculable dirac neutrino mass. Phys Rev Lett. (1987) 58:1600. doi: 10.1103/PhysRevLett.58.1600

65. Babu KS, He XG. Dirac neutrino masses as two loop radiative corrections. Mod Phys Lett A (1989) 4:61. doi: 10.1142/S0217732389000095

66. Babu KS, Mohapatra RN. CP violation in seesaw models of quark masses. Phys Rev Lett. (1989) 62:1079. doi: 10.1103/PhysRevLett.62.1079

67. Babu KS, Mohapatra RN. A solution to the strong CP problem without an axion. Phys Rev D (1990) 41:1286.

68. Balakrishna BS. Fermion mass hierarchy from radiative corrections. Phys Rev Lett. (1988) 60:1602. doi: 10.1103/PhysRevLett.60.1602

69. Balakrishna BS, Mohapatra RN. Radiative fermion masses from new physics at tev scale. Phys Lett $B$ (1989) 216:349-52. doi: 10.1016/0370-2693(89)91129-5

70. Balakrishna BS, Kagan AL, Mohapatra RN. Quark mixings and mass hierarchy from radiative corrections. Phys Lett B (1988) 205:345-52. doi: 10.1016/0370-2693(88)91676-0

71. Mohapatra RN, Zhang Y. TeV scale universal seesaw, vacuum stability and heavy higgs. JHEP (2014) 06:072. arXiv:1401.6701.

72. Dev PSB, Mohapatra RN, Zhang Y. Quark seesaw, vectorlike fermions and diphoton excess. JHEP (2016) 2:186. doi: 10.1007/JHEP02(2016)186

73. Deppisch FF, Hati C, Patra S, Pritimita P, Sarkar U. Implications of the diphoton excess on left?right models and gauge unification. Phys Lett B (2016) 757:223-30. doi: 10.1016/j.physletb.2016.03.081

74. Gabrielli E, Marzola L, Raidal M. Radiative yukawa couplings in the simplest left-right symmetric model. Phys Rev D (2017) 95:035005. doi: 10.1103/PhysRevD.95.035005

75. Patra S. Neutrinoless double beta decay process in left-right symmetric models without scalar bidoublet. Phys Rev D (2013) 87:015002. arXiv:1212.0612.

76. Guadagnoli D, Mohapatra RN, Sung I. Gauged flavor group with leftright symmetry. JHEP (2011) 04:093. arXiv:1103.4170. doi: 10.1007/ JHEP04(2011)093

77. Joshi GC, Volkas RR. Extended weak isospin and fermion masses in a unified model. Phys Rev D (1992) 45:1711-9. doi: 10.1103/PhysRevD.45.1711

78. Mohapatra RN, Vergados JD. A new contribution to neutrinoless double beta decay in gauge models. Phys Rev Lett. (1981) 47:1713-6. doi: 10.1103/PhysRevLett.47.1713

79. Hirsch M, Klapdor-Kleingrothaus HV, Panella O. Double beta decay in left-right symmetric models. Phys Lett B (1996) 374:7-12. doi: 10.1016/0370-2693(96)00185-2

80. Tello V, Nemevsek M, Nesti F, Senjanovic G, Vissani F. Left-right symmetry: from LHC to neutrinoless double beta decay. Phys Rev Lett. (2011) 106:151801. doi: 10.1103/PhysRevLett.106.151801

81. Chakrabortty J, Devi HZ, Goswami S, Patra S. Neutrinoless double- $\beta$ decay in TeV scale Left-Right symmetric models. JHEP (2012) 08:008. arXiv:hepph/9602306.

82. Barry J, Rodejohann W. Lepton number and flavour violation in TeVscale left-right symmetric theories with large left-right mixing. JHEP (2013) 09:153. doi: 10.1007/JHEP09(2013)153

83. Bhupal Dev PS, Goswami S, Mitra M, Rodejohann W. Constraining neutrino mass from neutrinoless double beta decay. Phys Rev D (2013) 88:091301. arXiv:1305.0056.
84. Ge SF, Lindner M, Patra S. New physics effects on neutrinoless double beta decay from right-handed current. JHEP (2015) 10:077. arXiv:1508.07286.

85. Awasthi RL, Dev PSB, Mitra M. Implications of the diboson excess for neutrinoless double beta decay and lepton flavor violation in TeV scale left right symmetric model. Phys Rev D (2016) 93:011701. doi: 10.1103/PhysRevD.93.011701

86. Huang WC, Lopez-Pavon J. On neutrinoless double beta decay in the minimal left-right symmetric model. Eur Phys J C (2014) 74:2853. doi: 10.1140/epjc/s10052-014-2853-z

87. Bhupal Dev PS, Goswami S, Mitra M. TeV scale left-right symmetry and large mixing effects in neutrinoless double beta decay. Phys Rev D (2015) 91:113004. doi: 10.1103/PhysRevD.91.113004

88. Borah D, Dasgupta A. Neutrinoless double beta decay in Type I+II seesaw models. JHEP (2015) 11:208. doi: 10.1007/JHEP11(2015)208

89. Bambhaniya G, Dev PSB, Goswami S, Mitra M. The scalar triplet contribution to lepton flavour violation and neutrinoless double beta decay in left-right symmetric model. JHEP (2016) 04:046. arXiv:1512.00440. doi: 10.1007/JHEP04(2016)046

90. Gu PH. Neutrinoless double beta decay in the left-right symmetric models for linear seesaw. JHEP (2016) 09:152. doi: 10.1007/JHEP09(2016)152

91. Borah D, Dasgupta A. Charged lepton flavour violcxmation and neutrinoless double beta decay in left-right symmetric models with type I+II seesaw. JHEP (2016) 07:022. arXiv:1606.00378.

92. Awasthi RL, Dasgupta A, Mitra M. Limiting the effective mass and new physics parameters from $0 v \beta \beta$. Phys Rev D (2016) 94:073003. arXiv:1607.03835.

93. Halprin A, Petcov ST, Rosen SP. Effects of light and heavy majorana neutrinos in neutrinoless double beta decay. Phys Lett B (1983) 125:335-8. doi: 10.1016/0370-2693(83)91296-0

94. Gonzalez-Garcia MC, Maltoni M, Salvado J, Schwetz T. Global fit to three neutrino mixing: critical look at present precision. JHEP (2012) 12:123. doi: 10.1007/JHEP12(2012)123

95. Meroni A, Petcov ST, Simkovic F. Multiple CP non-conserving mechanisms of $(\beta \beta)_{0 v}$-decay and nuclei with largely different nuclear matrix elements. JHEP (2013) 02:025. arXiv:1212.1331.

96. Gando A, Gando Y, Hachiya T, Hayashi A, Hayashida S, Ikeda $\mathrm{H}$, et al. Search for Majorana Neutrinos near the Inverted Mass Hierarchy Region with KamLAND-Zen. Phys Rev Lett. (2016) 117:082503. doi: 10.1103/PhysRevLett.117.082503 [Addendum: Phys. Rev. Lett. 117: 109903 (2016)].

97. Agostini M, Bakalyarov AM, Balata M, Barabanov I, Baudis L, Bauer C, et al. Searching for neutrinoless double beta decay with GERDA. In: 15th International Conference on Topics in Astroparticle and Underground Physics (TAUP 2017) (Sudbury, ON). Available online at: http://inspirehep.net/ record/1632043/files/arXiv:1710.07776.pdf

98. Albert J. Status and results from the EXO collaboration. EPJ Web Conf. (2014) 66:08001. doi: 10.1051/epjconf/20146608001

99. Mertens S. Status of the KATRIN Experiment and Prospects to Search for keV-mass Sterile Neutrinos in Tritium $\beta$-decay. Phys Procedia. (2015) 61:267-73. doi: 10.1016/j.phpro.2014.12.043

100. Ade PAR, Aghanim N, Arnaud M, Ashdown M, Aumont J, Baccigalupi C, et al. Planck 2015 Results. XIII. Cosmological Parameters (2015). 594:A13. arXiv:1502.01589. doi: 10.1051/0004-6361/201525830

101. Sakharov AD. Violation of CP Invariance, c Asymmetry, and Baryon Asymmetry of the Universe. Pisma Zh Eksp Teor Fiz. (1967) 5:32-5.

102. 't Hooft G. Symmetry breaking through bell-jackiw anomalies. Phys Rev Lett. (1976) 37:8-11. doi: 10.1103/PhysRevLett.37.8

103. 't Hooft G. Computation of the quantum effects due to a four-dimensional pseudoparticle. Phys Rev D (1976) 14:3432-50. [Erratum: Phys. Rev. D18, 2199(1978)]. doi: 10.1103/PhysRevD.14.3432

104. Kuzmin VA, Rubakov VA, Shaposhnikov ME. On the anomalous electroweak baryon number nonconservation in the early universe. Phys Lett B (1985) 155:36.

105. Arnold PB, Son D, Yaffe LG. The Hot baryon violation rate is O (alpha- $\mathrm{w}^{\star \star} 5$

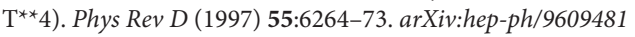

106. Arnold PB, Son DT, Yaffe LG. Effective dynamics of hot, soft nonAbelian gauge fields. Color conductivity and $\log (1 /$ alpha) effects. Phys Rev D (1999) 59:105020. 
107. Bodeker D. On the effective dynamics of soft nonAbelian gauge fields at finite temperature. Phys Lett B (1998) 426:351-60.

108. Bodeker D. From hard thermal loops to Langevin dynamics. Nucl Phys B (1999) 559:502-38.

109. Khlebnikov SYu, Shaposhnikov ME. The statistical theory of anomalous fermion number nonconservation. Nucl Phys B (1988) 308:885-912.

110. Harvey JA, Turner MS. Cosmological baryon and lepton number in the presence of electroweak fermion number violation. Phys Rev D (1990) 42:3344-9.

111. Sarkar U. Particle and Astroparticle Physics. New York, NY: Taylor \& Francis (2008). Available online at: http://www.crcpress.com/product/isbn/ 9781584889311.

112. Langacker P, Peccei RD, Yanagida $T$. Invisible axions and light neutrinos: are they connected? Mod Phys Lett A (1986) 1:541. doi: 10.1142/S0217732386000683

113. Luty MA. Baryogenesis via leptogenesis. Phys Rev D (1992) 45:455-65. doi: 10.1103/PhysRevD.45.455

114. Murayama H, Suzuki H, Yanagida T, Yokoyama J. Chaotic inflation and baryogenesis by right-handed sneutrinos. Phys Rev Lett. (1993) 70:1912-5. doi: 10.1103/PhysRevLett.70.1912

115. Acker A, Kikuchi H, Ma E, Sarkar U. CP violation and leptogenesis. Phys Rev $D$ (1993) 48:5006-8. arXiv:hep-ph/9305290.

116. Buchmuller W, Plumacher M. Baryon asymmetry and neutrino mixing. Phys Lett B (1996) 389:73-7. arXiv:hep-ph/9608308. doi: 10.1016/S0370-2693(96)01232-4

117. Flanz M, Paschos EA, Sarkar U. Baryogenesis from a lepton asymmetric universe. Phys Lett B (1995) 345:248-52. arXiv:hep-ph/9411366. [Erratum: Phys. Lett. B382, 447 (1996)]. doi: 10.1016/0370-2693(94)01 $555-\mathrm{Q}$

118. Flanz M, Paschos EA, Sarkar U, Weiss J. Baryogenesis through mixing of heavy Majorana neutrinos. Phys Lett B (1996) 389:693-9. arXiv:hepph/9607310. doi: 10.1016/S0370-2693(96)80011-6

119. Pilaftsis A. CP violation and baryogenesis due to heavy Majorana neutrinos. Phys Rev D (1997) 56:5431-51. arXiv:hep-ph/9707235. doi: 10.1103/ PhysRevD.56.5431

120. Pilaftsis $\mathrm{A}$. Resonant $\mathrm{CP}$ violation induced by particle mixing in transition amplitudes. Nucl Phys B (1997) 504:61-107. arXiv:hep-ph/9702393. doi: 10.1016/S0550-3213(97)00469-0

121. Roulet E, Covi L, Vissani F. On the CP asymmetries in Majorana neutrino decays. Phys Lett B (1998) 424:101-5. arXiv:hep-ph/9712468. doi: 10.1016/ S0370-2693(98)00135-X

122. Buchmuller W, Plumacher M. CP asymmetry in Majorana neutrino decays. Phys Lett B (1998) 431:354-62. arXiv:hep-ph/9710460. doi: 10.1016/S03702693(97)01548-7

123. Flanz M, Paschos EA. Further considerations on the CP asymmetry in heavy Majorana neutrino decays. Phys Rev B (1998) 58:113009. arXiv:hepph/9805427. doi: 10.1103/PhysRevD.58.113009

124. Hambye T, March-Russell J, West SM. TeV scale resonant leptogenesis from supersymmetry breaking. JHEP (2004) 07:070. arXiv:hep-ph/0403183. doi: $10.1088 / 1126-6708 / 2004 / 07 / 070$

125. Pilaftsis A, Underwood TEJ. Resonant leptogenesis. Nucl Phys B (2004) 692:303-45. arXiv:hep-ph/0309342. doi: 10.1016/j.nuclphysb.2004.05.029

126. Rangarajan R, Mishra H. Leptogenesis with heavy Majorana neutrinos revisited. Phys Rev D (2000) 61:043509. arXiv:hep-ph/9908417.

127. Davidson S, Ibarra A. A Lower bound on the right-handed neutrino mass from leptogenesis. Phys Lett B (2002) 535:25-32. arXiv:hep-ph/0202239. doi: 10.1016/S0370-2693(02)01735-5

128. Pilaftsis A, Underwood TEJ. Electroweak-scale resonant leptogenesis. Phys Rev D (2005) 72:113001. arXiv:hep-ph/0506107. doi: 10.1103/PhysRevD.72. 113001

129. Bhupal Dev PS, Millington P, Pilaftsis A, Teresi D. Flavour covariant transport equations: an application to resonant leptogenesis. Nucl Phys B (2014) 886:569-664. arXiv:1404.1003. doi: 10.1016/j.nuclphysb.2014. 06.020

130. Hambye T, Senjanovic G. Consequences of triplet seesaw for leptogenesis. Phys Lett B (2004) 582:73-81. arXiv:hep-ph/0307237. doi: 10.1016/j.physletb. 2003.11.061
131. Hallgren $\mathrm{T}$, Konstandin $\mathrm{T}$, Ohlsson $\mathrm{T}$. Triplet leptogenesis in left-right symmetric seesaw models. JCAP (2008) 0801:014. arXiv:0710.2408.

132. Lavignac $S$, Schmauch B. Flavour always matters in scalar triplet leptogenesis. JHEP (2015) 05:124. arXiv:1503.00629. doi: 10.1007/JHEP05(2015)124

133. Hambye T, Raidal M, Strumia A. Efficiency and maximal CP-asymmetry of scalar triplet leptogenesis. Phys Lett B (2006) 632:667-74. arXiv:hepph/0510008. doi: 10.1016/j.physletb.2005.11.007

134. Strumia A. Sommerfeld corrections to type-II and III leptogenesis. Nucl Phys B (2009) 809:308-17. arXiv:0806.1630. doi: 10.1016/j.nuclphysb.2008.10.007

135. Aristizabal Sierra D, Dhen M, Hambye T. Scalar triplet flavored leptogenesis: a systematic approach. JCAP (2014) 1408:003. arXiv:1401.4347. doi: 10.1088/ $1475-7516 / 2014 / 08 / 003$

136. Khachatryan V, Sirunyan AM, Tumasyan A, Adam W, Bergauer T, Dragicevic M, et al. Search for heavy neutrinos and $\mathrm{W}$ bosons with righthanded couplings in proton-proton collisions at $\sqrt{s}=8 \mathrm{TeV}$. Eur Phys J C (2014) 74:3149. arXiv:1407.3683. doi: 10.1140/epjc/s10052-014-3149-z

137. Deppisch FF, Gonzalo TE, Patra S, Sahu N, Sarkar U. Signal of righthanded charged gauge bosons at the LHC? Phys Rev D (2014) 90:053014. arXiv:1407.5384. doi: 10.1103/PhysRevD.90.053014

138. Deppisch FF, Gonzalo TE, Patra S, Sahu N, Sarkar U. Double beta decay, lepton flavor violation, and collider signatures of left-right symmetric models with spontaneous D-parity breaking. Phys Rev D (2015) 91:015018. arXiv:1410.6427. doi: 10.1103/PhysRevD.91.015018

139. Gluza J, Jelinski T. Heavy neutrinos and the pp?lljj CMS data. Phys Lett B (2015) 748:125-31. arXiv:1504.05568. doi: 10.1016/j.physletb.2015.06.077

140. Aad G, Abbott B, Abdallah J, Abdinov O, Aben R, Abolins M, et al. Search for high-mass diboson resonances with boson-tagged jets in proton-proton collisions at $\sqrt{s}=8 \mathrm{TeV}$ with the ATLAS detector. JHEP (2015) 12:055. arXiv:1506.00962. doi: 10.1007/JHEP12(2015)055

141. Brehmer J, Hewett J, Kopp J, Rizzo T, Tattersall J. Symmetry restored in dibosons at the LHC? JHEP (2015) 10:182. arXiv:1507.00013.

142. Dobrescu BA, Liu Z. W' Boson near 2 TeV: Predictions for Run 2 of the LHC. Phys Rev Lett. (2015) 115:211802. arXiv:1506.06736. doi: 10.1103/ PhysRevLett.115.211802

143. Bhupal Dev PS, Mohapatra RN. Unified explanation of the eejj, diboson and dijet resonances at the LHC. Phys Rev Lett. (2015) 115:181803. doi: 10.1103/PhysRevLett.115.181803

144. Das A, Nagata N, Okada N. Testing the 2-TeV Resonance with Trileptons. JHEP (2016) 03:049. arXiv:1601.05079. doi: 10.1007/JHEP03(2016)049

145. Ma E, Sarkar S, Sarkar U. Scale of $\mathrm{SU}(2)(\mathrm{R})$ symmetry breaking and leptogenesis. Phys Lett B (1999) 458:73-8. arXiv:hep-ph/9812276.

146. Deppisch FF, Harz J, Hirsch M. Falsifying high-scale leptogenesis at the LHC. Phys Rev Lett. (2014) 112:221601. doi: 10.1103/PhysRevLett.112.221601

147. Deppisch FF, Harz J, Hirsch M, Huang WC, Pas H. Falsifying high-scale baryogenesis with neutrinoless double beta decay and lepton flavor violation. Phys Rev D (2015) 92:036005. doi: 10.1103/PhysRevD.92.036005

148. Frere JM, Hambye T, Vertongen G. Is leptogenesis falsifiable at LHC? JHEP (2009) 01:051. arXiv:0806.0841.

149. Bhupal Dev PS, Lee $\mathrm{CH}$, Mohapatra RN. TeV scale lepton number violation and baryogenesis. J Phys Conf Ser. (2015) 631:012007. doi: 10.1088/1742-6596/631/1/012007

150. Bhupal Dev PS, Lee CH, Mohapatra RN. Leptogenesis constraints on the mass of right-handed gauge bosons. Phys Rev D (2014) 90:095012. arXiv:1408.2820.

151. Dhuria M, Hati C, Rangarajan R, Sarkar U. The eejj Excess Signal at the LHC and Constraints on Leptogenesis. JCAP (2015) 1509:035. arXiv:1502.01695. doi: 10.1088/1475-7516/2015/09/035

152. Dhuria M, Hati C, Rangarajan R, Sarkar U. Falsifying leptogenesis for a $\mathrm{TeV}$ scale $W_{R}^{ \pm}$at the LHC. Phys Rev D (2015) 92:031701. arXiv:1503.07198.

153. Doi M. Lepton number violating e- W+ $\rightarrow$ e+ W- and e- e- $\rightarrow$ W- Wprocesses in the left-right gauge model. Prog Theor Phys. (1999) 101:639-60. doi: 10.1143/PTP.101.639

154. Olive KA, Agashe K, Amsler C, Antonelli M, Arguin JF, Asner DM, et al. Review of particle physics. Chin Phys C (2014) 38:090001.

155. Mohapatra RN, Marshak RE. Phenomenology of neutron oscillations. Phys Lett B (1980) 94:183-6. doi: 10.1016/0370-2693(80)90853-9 [Erratum: Phys. Lett. B96, 444 (1980)]. 
156. Phillips DG II, Snow WM, Babu K, Banerjee S, Baxter DV, Berezhiani Z, et al. Neutron-antineutron oscillations: theoretical status and experimental prospects. Phys Rept. (2016) 612:1-45. doi: 10.1016/j.physrep.2015. 11.001

157. Pati JC, Salam A, Sarkar U. Delta B $=-$ Delta L, neutron $\rightarrow$ e- pi+, e- K+, mu- pi+ and mu- K+ DECAY modes in SU(2)-L X SU(2)-R X SU(4)-C or SO(10). Phys Lett B (1983) 133:330-6.

158. Dhuria M, Hati C, Rangarajan R, Sarkar U. Explaining the CMS eejj and $e$ missing $\mathrm{p}_{\mathrm{T}} \mathrm{jj}$ excess and leptogenesis in superstring inspired $E_{6}$ models. Phys Rev D (2015) 91:055010. arXiv:1501. 04815.
Conflict of Interest Statement: The authors declare that the research was conducted in the absence of any commercial or financial relationships that could be construed as a potential conflict of interest.

Copyright $\odot 2018$ Hati, Patra, Pritimita and Sarkar. This is an open-access article distributed under the terms of the Creative Commons Attribution License (CC $B Y)$. The use, distribution or reproduction in other forums is permitted, provided the original author(s) and the copyright owner are credited and that the original publication in this journal is cited, in accordance with accepted academic practice. No use, distribution or reproduction is permitted which does not comply with these terms. 